\title{
COMPLEX VALUED ANALYTIC TORSION AND DYNAMICAL ZETA FUNCTION ON LOCALLY SYMMETRIC SPACES
}

\author{
SHU SHEN
}

\begin{abstract}
We show that the Ruelle dynamical zeta function on a closed odd dimensional locally symmetric space twisted by an arbitrary flat vector bundle has a meromorphic extension to the whole complex plane and that its leading term in the Laurent series at the zero point is related to the regularised determinant of the flat Laplacian of Cappell-Miller. When the flat vector bundle is close to an acyclic and unitary one, we show that the dynamical zeta function is regular at the zero point and that its value is equal to the complex valued analytic torsion of Cappell-Miller. This generalises author's previous results for unitarily flat vector bundles as well as Müller and Spilioti's results on hyperbolic manifolds.
\end{abstract}

\section{CONTENTS}

Introduction

$\begin{array}{ll}\text { 1. The generalised Laplacian } & 11\end{array}$

1.1. The spectral theory of a generalised Laplacian 11

$\begin{array}{ll}\text { 1.2. The semigroup of a generalised Laplacian } & 12\end{array}$

$\begin{array}{ll}\text { 1.3. The regularised determinant } & 15\end{array}$

$\begin{array}{ll}\text { 1.4. Flat vector bundles } & 15\end{array}$

$\begin{array}{ll}\text { 1.5. A generalised Laplacian and flat vector bundles } & 17\end{array}$

1.6. Flat Laplacian and the Cappell-Miller analytic torsion 20

2. Müller's Selberg trace formula 22

2.1. Real reductive groups $\quad 22$

2.2. The Casimir operator 24

2.3. The symmetric space 24

2.4. Semisimple elements 25

2.5. Semisimple orbital integrals 26

2.6. A discrete subgroup of $G \quad 26$

3. Fundamental Cartan subalgebra and related constructions 28

3.1. A fundamental Cartan subalgebra 28

$\begin{array}{ll}\text { 3.2. A splitting of } \mathfrak{g} & 29\end{array}$

$\begin{array}{lll}\text { 3.3. A root system of }(\mathfrak{h}, \mathfrak{g}) & 30\end{array}$

$\begin{array}{ll}\text { 3.4. A lifting property } & 31\end{array}$

4. The Ruelle dynamical zeta functions for arbitrary twist 32

Date: September 9, 2020.

2020 Mathematics Subject Classification. 58J20,58J52,11F72,11M36,37C30.

Key words and phrases. Index theory and related fixed point theorems, analytic torsion, Selberg trace formula, Ruelle dynamical zeta function.

I am indebted to Xiaolong Han, Xiaonan Ma, Jianqing Yu, and Weiping Zhang for reading the preliminary version of this article and for useful discussions. 
5. The Selberg zeta function for arbitrary twist 34

5.1. The structure of the reductive group $G$ with $\delta(G)=1$

5.2. The Selberg zeta function 36

5.3. Proof of (5.21) for $\sigma \gg 1$

5.4. Proof of Theorem $4.3 \quad 38$

6. A cohomological formula for $r_{j} \quad 39$

6.1. Preliminary on the representation theory 40

6.2. The right regular representation on $C^{\infty}\left(\Gamma \backslash G, \widehat{p}^{*} F\right)$

6.3. Formulas for $r_{\eta, \rho}$ and $r_{j} \quad 44$

7. An extension to orbifolds 47

7.1. Müller's Selberg trace formula on locally symmetric orbifolds 47

7.2. The Ruelle zeta functions on locally symmetric orbifolds 48

$\begin{array}{ll}\text { References } & 48\end{array}$

\section{INTRODUCTION}

The purpose of this article is to study the relation between the complex valued analytic torsion of Cappell-Miller and the value at the zero point of the Ruelle dynamical zeta function associated to a flat vector bundle, which is not necessarily unitarily flat, on a closed odd dimensional locally symmetric space of reductive type.

Let $Z$ be a smooth closed manifold. Let $F$ be a complex flat vector bundle on $Z$. Let $H^{\bullet}(Z, F)$ be the cohomology of the sheaf of locally constant sections of $F$. We assume $H^{\cdot}(Z, F)=0$.

Let $g^{T Z}, g^{F}$ be the metrics on $T Z$ and $F$. The analytic torsion is a real positive number introduced by Ray and Singer [RaSi71]. It is a spectral invariant defined by the Hodge Laplacian associated with $g^{T Z}, g^{F}$. If $\operatorname{dim} Z$ is odd, they showed that the analytic torsion does not depend on the metric data, and conjectured that, if $F$ is unitarily flat (i.e., the holonomy representation of $F$ is unitary), there is an equality between the analytic torsion and its topological counterpart, the Reidemeister torsion [Re35, Fra35, dR50].

Ray and Singer's conjecture was proved by Cheeger [Ch79] and Müller [Mü78], and is now known under the name of Cheeger-Müller Theorem. Bismut-Zhang [BZ92] and Müller [Mü93] simultaneously considered generalisations of this result. Müller [Mü93] extended his result to odd dimensional oriented manifold and only $\operatorname{det} F$ is required to be unitarily flat. Using the Witten deformation [W82], Bismut and Zhang [BZ92] generalised the original Cheeger-Müller theorem to arbitrary flat vector bundles with arbitrary Hermitian metrics on a manifold with arbitrary dimension.

On the other hand, Turaev [T89, FaT00] generalised the concept of the Reidemeister torsion to a complex valued invariant whose absolute value provides the original Reidemeister torsion, with the help of the so-called Euler structure.

The analytic counterpart of the Turaev torsion was introduced by BravermanKappeler, Burghelea-Haller, and Cappell-Miller separately. In [BrKa08, BrKa07], 
using the signature operator, Braverman and Kappeler defined what they called the refined analytic torsion for flat vector bundles on odd dimensional manifolds, and showed that it is equal to the Turaev torsion up to a multiplication by a complex number of absolute value one. In [BuH07, BuH08], Burghelea and Haller defined a generalised analytic torsion associated to a nondegenerate symmetric bilinear form on a flat vector bundle over an arbitrary dimensional manifold. Following the idea of [BZ92], Su and Zhang [SuZ08] showed that the BurgheleaHaller torsion coincides with the square of the Turaev torsion. In [BuH10], using different methods, Burghelea and Haller proved Su-Zhang's result up to the sign, in the case of odd dimension manifolds. In [CMil10], with the help of the flat Laplacian, Cappell-Miller introduced another version of the analytic torsion associated to a flat vector bundle on an arbitrary dimensional manifold without assuming the existence of the nondegenerate symmetric bilinear form. By adopting the proof of Su-Zhang [SuZ08], Cappell-Miller showed that their torsion invariant is equal to its topological counterpart when the manifold is oriented and is of odd dimension.

Milnor [Mi68a] initiated the study of the relation between the torsion invariant and a dynamical system. Fried [F86] showed that the Ray-Singer analytic torsion of an acyclic unitarily flat vector bundle on a closed odd dimensional hyperbolic manifold is equal to the value at the zero point of the Ruelle dynamical zeta function of the geodesic flow. He conjectured [F87, p. 66, Conjecture] that similar results should hold true for more general flows. In [Sh18], following previous contributions by Moscovici-Stanton [MoSt91], using Bismut's orbital integral formula [B11], the author affirmed the Fried conjecture for geodesic flows on closed odd dimensional locally symmetric manifolds equipped with a unitarily flat vector bundle. In [ShY17], the authors made a further generalisation to closed odd dimensional locally symmetric orbifolds. We refer the reader to [Ma19] for an introduction to the technique used in [Sh18].

The Fried conjecture for arbitrary flat vector bundle is recently considered by Spilioti [Sp20a, Sp18, Sp20b] and by Müller [Mü20] for closed odd dimensional hyperbolic manifolds. When the underlying manifold is hyperbolic, Spilioti [Sp20a, Sp18] showed that the Ruelle dynamical zeta function has a meromorphic extension to $\mathbf{C}$. Müller [Mü20] related its leading coefficient in the Laurent series at the zero point to the complex valued analytic torsion of Cappell-Miller. When the flat vector bundle is close to an acyclic and unitary one, it is shown [Mï20, Sp20b] that the dynamical zeta function is regular at the zero point and its value is equal to the Cappell-Miller analytic torsion.

In this article, we extend the above results of Müller and Spilioti to closed odd dimensional locally symmetric spaces of arbitrary ranks.

We refer the reader to [Sh19] for a survey on the Fried conjecture on different flows, like Morse-Smale flow [ShY18] and Anosov flow [DGRSh20].

Now, we will describe our results in more detail, and explain the techniques used in their proofs.

0.1. The analytic torsions of Ray-Singer and Cappell-Miller. Let $Z$ be a smooth closed manifold of dimension $m$, and let $\left(F, \nabla^{F}\right)$ be a complex flat vector 
bundle of rank $r$ on $Z$ with flat connection $\nabla^{F}$. Let $\Gamma$ be the fundamental group of $Z$. Let $\rho: \Gamma \rightarrow \mathrm{GL}_{r}(\mathbf{C})$ be the holonomy representation of $F$. Let $\left(\Omega^{\bullet}(Z, F), d^{Z}\right)$ be the de Rham complex with values in $F$. Let $H^{\bullet}(Z, F)$ be the corresponding cohomology.

If $X$ is the universal cover of $Z$, if $\left(\Omega^{\bullet}(X) \otimes \mathbf{C}^{r}\right)^{\Gamma}$ is the space of $\mathbf{C}^{r}$-valued $\Gamma$ invariant forms on $X$, then

$$
\Omega^{\bullet}(Z, F)=\left(\Omega^{\bullet}(X) \otimes \mathbf{C}^{r}\right)^{\Gamma} .
$$

Let $g^{T Z}$ be a Riemannian metric on $T Z$. Let $\square^{Z}$ be the associated flat Laplacian acting on $\Omega^{\cdot}(Z, F)$. It can be obtained by the restriction of the Hodge Laplacian on $X$ to the $\Gamma$-invariant subspace (0.1).

If $F$ has a flat metric $g^{F}, \square^{Z}$ is just the Hodge Laplacian associated with $g^{T Z}, g^{F}$. By Hodge theory, we have

$$
\operatorname{ker} \square^{Z}=H^{\cdot}(Z, F) .
$$

The Ray-Singer analytic torsion is a positive real number defined by the following weighted product of the zeta regularised determinants

$$
T_{\mathrm{RS}}(F)=\prod_{i=1}^{m} \operatorname{det}^{*}\left(\left.\square^{Z}\right|_{\Omega^{i}(Z, F)}\right)^{(-1)^{i} i / 2} .
$$

Formally, det* means the product of non zero eigenvalues counted with multiplicities. By a fundamental result of Ray-Singer [RaSi71] (see also [BZ92, Theorem $0.1]$ ), if $\operatorname{dim} Z$ is odd and if $H^{\bullet}(Z, F)=0, T_{\mathrm{RS}}(F)$ is independent of $g^{T Z}$ and $g^{F}$.

For general $F, \square^{Z}$ is a second order elliptic differential operator, which is not necessarily self-adjoint. Still $\square^{Z}$ has a Hodge-like theory. Namely, if $\Omega_{0}^{\cdot}(Z, F)$ is the characteristic space of $\square^{Z}$ associated with the eigenvalue 0 , then $\left(\Omega_{0}^{\circ}(Z, F), d^{Z}\right)$ is a finite dimensional complex such that

$$
H^{\bullet}\left(\Omega_{0}^{\cdot}(Z, F), d^{Z}\right) \simeq H^{\bullet}(Z, F) .
$$

By (0.4), if $\square^{Z}$ is invertible, then $H^{\bullet}(Z, F)=0$. In this case, the Cappell-Miller torsion $T_{\mathrm{CM}}(F) \in \mathbf{C}$ is a non vanishing complex number given by the following weighted product of the zeta regularised determinants (see Section 1.3 for a definition)

$$
T_{\mathrm{CM}}(F)=\prod_{i=1}^{m} \operatorname{det}^{*}\left(\left.\square^{Z}\right|_{\Omega^{i}(Z, F)}\right)^{(-1)^{i} i} .
$$

Note that with our convention, Cappell-Miller's original definition [CMil10] corresponds to the inverse of $T_{\mathrm{CM}}(F)$.

For a general $F$, the Cappell-Miller torsion $T_{\mathrm{CM}}(F)$ is an element in the dual of the determinant line of the graded space $H^{\bullet}\left(Z, F \oplus F^{*}\right)$ (see [CMil10, Section 8]). It has been shown that [CMil10, Theorem 8.3] if $\operatorname{dim} Z$ is odd, $T_{\mathrm{CM}}(F)$ does not depend on $g^{T Z}$ and is a topological invariant.

0.2. The dynamical zeta function of Ruelle. Let us recall the definition of the Ruelle dynamical zeta function for geodesic flows introduced by Fried [F87, Section 5] (see also [Sh19, Section 2]).

Let $\left(Z, g^{T Z}\right)$ be a connected manifold with nonpositive sectional curvature. Let $[\Gamma]$ be the set of the conjugacy classes of the fundamental group $\Gamma$. For $[\gamma] \in[\Gamma]$, 
let $B_{[\gamma]}$ be the set of closed geodesics in the free homotopy class associated to $[\gamma]$. It is easy to see that all the elements in $B_{[\gamma]}$ have the same length $\ell_{[\gamma]}$.

For simplicity, assume that all the $B_{[\gamma]}$ are smooth finite dimensional submanifolds of the loop space of $Z$. This is the case if $\left(Z, g^{T Z}\right)$ has a negative sectional curvature or if $Z$ is locally symmetric. If $\gamma=1, B_{[1]}=Z$ is the space of trivial geodesics. If $\gamma \neq 1$, the group $\mathbb{S}^{1}$ acts locally freely on $B_{[\gamma]}$ by rotation, so that $B_{[\gamma]} / \mathbb{S}^{1}$ is an orbifold. Let $\chi_{\mathrm{orb}}\left(B_{[\gamma]} / \mathbb{S}^{1}\right) \in \mathbf{Q}$ be the orbifold Euler characteristic [Sa57]. Denote by

$$
m_{[\gamma]}=\left|\operatorname{ker}\left(\mathbb{S}^{1} \rightarrow \operatorname{Diff}\left(B_{[\gamma]}\right)\right)\right| \in \mathbf{N}^{*}
$$

the multiplicity of a generic element in $B_{[\gamma]}$. Let $\epsilon_{[\gamma]}= \pm 1$ be the Lefschetz index of the Poincaré return map along a closed geodesic in $B_{[\gamma]}$ induced by the geodesic flow (see [Sh19, (2.17)] for a precise definition). If $Z$ is locally symmetric, then $\epsilon_{[\gamma]}=1$.

Let $\rho: \Gamma \rightarrow \mathrm{GL}_{r}(\mathbf{C})$ be a representation of $\Gamma$. The formal dynamical zeta function of Ruelle is defined for $\sigma \in \mathbf{C}$ by

$$
R_{\rho}(\sigma)=\exp \left(\sum_{[\gamma] \in\left[\Gamma_{+}\right]} \epsilon_{[\gamma]} \operatorname{Tr}[\rho(\gamma)] \frac{\chi_{\text {orb }}\left(B_{[\gamma]} / \mathbb{S}^{1}\right)}{m_{[\gamma]}} e^{-\sigma \ell_{[\gamma]}}\right),
$$

where $\left[\Gamma_{+}\right]=[\Gamma]-\{1\}$ is the set of the non trivial conjugacy classes of $\Gamma$. We will say that the formal dynamical zeta function is well defined if $R_{\rho}(\sigma)$ is holomorphic for $\operatorname{Re}(\sigma) \gg 1$ and extends meromorphically to $\sigma \in \mathbf{C}$.

Observe that if $\left(Z, g^{T Z}\right)$ is of negative sectional curvature, then $B_{[\gamma]} \simeq \mathbb{S}^{1}$ and

$$
\chi_{\text {orb }}\left(B_{[\gamma]} / \mathbb{S}^{1}\right)=1 .
$$

In this case, if $\rho$ is a trivial representation, $R_{\rho}(\sigma)$ was recently shown to be well defined ${ }^{1}$ by Giulietti-Liverani-Pollicott [GiLPo13] and Dyatlov-Zworski [DyZw16]. Moreover, Dyatlov and Zworski [DyZw17] and Borns-Weil and Shen [BWSh20] showed that, if $\left(Z, g^{T Z}\right)$ is a negatively curved surface, the order of the zero of $R_{\rho}(\sigma)$ at $\sigma=0$ is related to the genus of $Z$. For general $\rho$, the proof of the meromorphic extension of $R_{\rho}$ is not particularly difficult. In particular, when 0 is not a resonance of the weight dynamical system associated to $\rho$, then $R_{\rho}(0)$ is well defined. In this case, Dang, Guillarmou, Rivière, and Shen [DGRSh20] showed that $R_{\rho}(0)$ remains unchanged under a small perturbation of the geodesic flow.

0.3. Results of Fried, Müller, and Spilioti on hyperbolic manifolds. Assume that $Z$ is a connected closed odd dimensional orientable hyperbolic manifold. Recall that $\left(F, \nabla^{F}\right)$ is a flat vector bundle on $Z$ with holonomy $\rho: \Gamma \rightarrow \mathrm{GL}_{r}(\mathbf{C})$.

If $F$ has a flat metric or equivalently $\rho$ is unitary, using the Selberg trace formula, Fried [F86, Theorem 3] showed that there exist explicit constants $C_{\rho} \in \mathbf{R}^{*}$ and $r_{\rho} \in \mathbf{Z}$ such that as $\sigma \rightarrow 0$,

$$
R_{\rho}(\sigma)=C_{\rho} T_{\mathrm{RS}}(F)^{2} \sigma^{r_{\rho}}+\mathscr{O}\left(\sigma^{r_{\rho}+1}\right) .
$$

\footnotetext{
${ }^{1}$ More precisely, the authors [GiLPo13, DyZw16] showed the meromorphic extension when the flow is Anosov.
} 
Moreover, if $H^{\cdot}(Z, F)=0$, then

$$
C_{\rho}=1, \quad r_{\rho}=0,
$$

so that

$$
R_{\rho}(0)=T_{\mathrm{RS}}(F)^{2}
$$

When $\rho$ is not unitary, in [Mü20, Theorem 1.1], Müller has shown a similar result. Namely, there exist explicit constants $C_{\rho} \in \mathbf{R}^{*}$ and $r_{\rho} \in \mathbf{Z}$ such that as $\sigma \rightarrow 0$,

$$
R_{\rho}(\sigma)=C_{\rho} \prod_{i=1}^{m} \operatorname{det}^{*}\left(\left.\square^{Z}\right|_{\Omega^{i}(Z, F)}\right)^{(-1)^{i} i}+\mathscr{O}\left(\sigma^{r_{\rho}+1}\right) .
$$

Moreover, the constants $C_{\rho}$ and $r_{\rho}$ depend only on the dimension of $\Omega_{0}^{*}(Z, F)$.

If $\rho$ is close to a unitary and acyclic representation of $\Gamma$ in the sense of $C^{0}$ ([BrKa08, Section 6.7], see also Section 1.6), by [BrKa08, Proposition 6.8] (see also [Mü20, Lemma 6.2]), we have $\Omega_{0}^{*}(Z, F)=0$. In this case, in [Mü20, Proposition 1.3], Müller obtained $C_{\rho}=1, r_{\rho}=0$, and

$$
R_{\rho}(0)=T_{\mathrm{CM}}(F) .
$$

Similar results were also obtained by Spilioti [Sp20b, Theorem 2].

0.4. The main result of the article. Let $G$ be a linear connected real reductive group [K86, p. 3], and let $\theta$ be the Cartan involution. Let $K$ be the maximal compact subgroup of the points of $G$ that are fixed by $\theta$. Let $\mathfrak{k}$ and $\mathfrak{g}$ be the Lie algebras of $K$ and $G$, and let $\mathfrak{g}=\mathfrak{p} \oplus \mathfrak{k}$ be the Cartan decomposition. Let $B$ be a nondegenerate bilinear symmetric form on $\mathfrak{g}$ which is invariant under the adjoint action of $G$ and under $\theta$. Assume that $B$ is positive on $\mathfrak{p}$ and negative on $\mathfrak{k}$. Set $X=G / K$. Then $\left.B\right|_{\mathfrak{p}}$ induces a Riemannian metric $g^{T X}$ on $X$, such that $\left(X, g^{T X}\right)$ has a parallel nonpositive sectional curvature.

Let $\Gamma \subset G$ be a discrete cocompact subgroup of $G$. Assume for the moment that $\Gamma$ is torsion free. Set $Z=\Gamma \backslash X$. Then $Z$ is a closed locally symmetric manifold, equipped with the induced Riemannian metric $g^{T Z}$. Let $\rho: \Gamma \rightarrow \mathrm{GL}_{r}(\mathbf{C})$ be a representation of $\Gamma$. Let $F=\Gamma \backslash\left(X \times \mathbf{C}^{r}\right)$ be the associated flat vector bundle on $Z$.

When $\rho$ is unitary, in $[\mathrm{F} 87$, p. 66 , Conjecture], Fried conjectured similar results (0.9)-(0.11) still hold. This conjecture was recently proved by the author [Sh18] and generalised by Shen-Yu [ShY17] on locally symmetric orbifolds where $\Gamma$ is no longer torsion free, following previous contributions by Moscovici-Stanton [MoSt91], using Bismut's orbital integral formula [B11].

The main result of this article concernes the case where $\rho$ is not always unitary. It generalises [F86, Theorem 3], [Mü20, Theorem 1.1, Proposition 1.3], [Sp20b, Theorem 2] to locally symmetric space, as well as [Sh18, Theorem 1.1] to non unitary twist. Recall that $\square^{Z}$ is the flat Laplacian on $\Omega^{\bullet}(Z, F)$.

Theorem 0.1. Assume that $\operatorname{dim} Z$ is odd. The following statements hold:

i) The dynamical zeta function $R_{\rho}(\sigma)$ is holomorphic for $\operatorname{Re}(\sigma) \gg 1$ and extends meromorphically to $\sigma \in \mathbf{C}$. 
ii) There exist explicit constants $C_{\rho} \in \mathbf{R}^{*}$ and $r_{\rho} \in \mathbf{Z}$ (see (5.33) and (5.35)) such that, when $\sigma \rightarrow 0$, we have

$$
R_{\rho}(\sigma)=C_{\rho}\left\{\prod_{i=1}^{m} \operatorname{det}^{*}\left(\left.\square^{Z}\right|_{\Omega^{i}(Z, F)}\right)^{(-1)^{i} i}\right\} \sigma^{r_{\rho}}+\mathscr{O}\left(\sigma^{r_{\rho}+1}\right) .
$$

iii) If $\rho$ is close enough (see (1.78)) to an acyclic and unitary representation of $\Gamma$, then $\square^{Z}$ is invertible and

$$
C_{\rho}=1
$$

$$
r_{\rho}=0
$$

so that

$$
R_{\rho}(0)=T_{\mathrm{CM}}(F) .
$$

We will also extend the above result to the case where $\Gamma$ is not torsion free. Then $Z$ is an orbifold and $F$ is a flat orbifold vector bundle. In this case the analytic torsion of Ray-Singer is still well-defined [Ma05, DaY17, ShY17], and we can define the Cappell-Miller torsion in a similar way. Also, the Ruelle zeta function $R_{\rho}(\sigma)$ was introduced in [ShY17].

Theorem 0.2. The statement of Theorem 0.1 holds when $Z=\Gamma \backslash G / K$ is an orbifold.

If the representation $\rho: \Gamma \rightarrow \mathrm{GL}_{r}(\mathbf{C})$ admits an invariant bilinear form, it induces a flat bilinear form $b_{F}$ on $F$. Since $b_{F}$ is flat, by (0.1), the Laplacian of Burghelea-Haller [BuH07, (26)] [SuZ08, (2.20)] coincides with the flat Laplacian $\square^{Z}$ of Cappell-Miller. In particular, we get the following corollary.

Corollary 0.3. If the representation $\rho: \Gamma \rightarrow \mathrm{GL}_{r}(\mathbf{C})$ admits an invariant bilinear form, the statements of Theorems 0.1 and 0.2 hold for the complexed valued analytic torsion of Burghelea-Haller.

\subsection{Moscovici-Stanton's vanishing theorem and Kazhdan's property $(\mathbf{T})$.}

Let $\delta(G) \in \mathbf{N}$ be the fundamental rank of $G$, i.e., the difference between the complex ranks of $G$ and $K$. Note that $\delta(G)$ and $\operatorname{dim} Z$ have the same parity.

If $\delta(G) \geqslant 3$, our theorem follows easily from an observation originally due to Moscovici-Stanton [MoSt91, Corollary 2.2, Remark 3.7] (see also Bismut [B11, Theorem 7.93]). More precisely,

$$
R_{\rho}(\sigma) \equiv 1, \quad \quad \prod_{i=1}^{m} \operatorname{det}^{*}\left(\left.\square^{Z}\right|_{\Omega^{i}(Z, F)}\right)^{(-1)^{i} i}=1 .
$$

When $\delta(G)=1$, Theorem 0.1 i) ii) is more significant (see [BMaZ11, BMaZ17] and also [MüPf13] when $\rho$ is the restriction of a representation of $G$ ). By the classification theory of real simple Lie algebras ([B11, Remark 7.9.2] and [Sh18, Theorem 6.15]), $\delta(G)=1$ is equivalent to

$$
\mathfrak{g}=\mathfrak{g}_{1} \oplus\left\{\begin{array}{l}
\mathbf{R} \\
\mathfrak{s l}_{3}(\mathbf{R}) \\
\mathfrak{s o}(p, q) \text { with } p \geqslant q, p q>1 \text { odd },
\end{array}\right.
$$

where $\mathfrak{g}_{1}$ is a real semisimple Lie algebra such that $\delta\left(\mathfrak{g}_{1}\right)=0$. Here, $\delta\left(\mathfrak{g}_{1}\right)$ is defined in an obvious way as $\delta(G)$. Note that $\mathfrak{s o}(1,1) \simeq \mathbf{R}$. However, we single out this case since $\mathbf{R}$ is not considered to be simple. 
Theorem 0.1 iii) makes sense only if $\Gamma$ has an acyclic and unitary representation and if such a representation is not isolated. A construction of acyclic and unitary representations can be founded in [FrN15]. Assume now that $\Gamma$ admits an acyclic and unitary representation. If $\Gamma$ has Kazhdan's property (T), by [Rap99], all unitary representations of $\Gamma$ are isolated. Therefore, Theorem 0.1 iii) gives interesting results only if $\Gamma$ does not have Kazhdan's property (T). By [BHdlVa08, Theorems 1.7.1 and 3.5.4], this is the case when $\mathfrak{g}$ contains a factor $\mathfrak{s o}(p, 1)$ or $\mathfrak{s u}(p, 1)$. Combining it with (0.18), we get

$$
\mathfrak{g}=\mathfrak{g}_{1} \oplus\left\{\begin{array}{l}
\mathbf{R} \\
\mathfrak{s o}(p, 1) \text { with } p \geqslant 3 \text { odd }
\end{array}\right.
$$

or

$$
\mathfrak{g}=\mathfrak{g}_{1} \oplus\left\{\begin{array} { l } 
{ \mathfrak { s u } ( s , 1 ) \text { with } s \geqslant 1 } \\
{ \mathfrak { s o } ( s , 1 ) \text { with } s \geqslant 2 \text { even } }
\end{array} \oplus \left\{\begin{array}{l}
\mathfrak{s l}_{3}(\mathbf{R}) \\
\mathfrak{s o}(p, q) \text { with } p \geqslant q>1, p q \text { odd }
\end{array}\right.\right.
$$

where $\mathfrak{g}_{1}$ is a real semisimple Lie algebra with $\delta\left(\mathfrak{g}_{1}\right)=0$ in both of the above two cases.

0.6. The proof of Theorem 0.1. Our proof of Theorem 0.1 is inspired by [Sh18]. As we have seen that we can reduce the proof to the case where $\delta(G)=1$. In this case, our proof relies on the Selberg zeta functions.

Assume now $\delta(G)=1$. Let $\mathfrak{t} \subset \mathfrak{k}$ be a Cartan subalgebra of $\mathfrak{k}$. Let $\mathfrak{h}=\mathfrak{z}(\mathfrak{t}) \subset \mathfrak{g}$ be the stabiliser of $\mathfrak{t}$ in $\mathfrak{g}$. By [K86, p. 129], $\mathfrak{h} \subset \mathfrak{g}$ is a $\theta$-invariant fundamental Cartan subalgebra of $\mathfrak{g}$. Let $\mathfrak{h}=\mathfrak{b} \oplus \mathfrak{t}$ be the Cartan decomposition of $\mathfrak{h}$. Note that $\operatorname{dim} \mathfrak{b}=\delta(G)=1$. Let $H \subset G$ be the associated Cartan subgroup of $G$.

Let $Z(\mathfrak{b}) \subset G$ be the stabiliser of $\mathfrak{b}$ in $G$ with Lie algebra $\mathfrak{z}(\mathfrak{b})$. Let $Z^{0}(\mathfrak{b})$ be the connected component of the identity in $Z(\mathfrak{b})$. Then $\mathfrak{z}(\mathfrak{b}), Z^{0}(\mathfrak{b})$ split

$$
\mathfrak{z}(\mathfrak{b})=\mathfrak{b} \oplus \mathfrak{m}, \quad Z^{0}(\mathfrak{b})=\exp (\mathfrak{b}) \times M
$$

where $M$ is a connected reductive subgroup of $G$ with Lie algebra $\mathfrak{m}$. Let $\mathfrak{m}=$ $\mathfrak{p}_{\mathfrak{m}} \oplus \mathfrak{k}_{\mathfrak{m}}$ be the Cartan decomposition of $\mathfrak{m}$. Let $\mathfrak{z}^{\perp}(\mathfrak{b}) \subset \mathfrak{g}$ be the orthogonal space of $\mathfrak{z}^{\perp}(\mathfrak{b})$ with respect to $B$.

We fix an orientation on $\mathfrak{b}$, whose choice is irrelevant. Let $\mathfrak{n} \subset \mathfrak{g}$ be the direct sum of the eigenspaces associated with the positive eigenvalues of a positive element in $\mathfrak{b}$. Then, $\mathfrak{n}$ is a Lie subalgebra of $\mathfrak{g}$ so that

$$
\mathfrak{z}^{\perp}(\mathfrak{b})=\mathfrak{n} \oplus \theta \mathfrak{n} .
$$

Let $\eta=\eta^{+}-\eta^{-}$be a virtual representation of $M$ acting on the finite dimensional complex vector spaces $E_{\eta}=E_{\eta}^{+}-E_{\eta}^{-}$such that

- the Casimir of $M$ acts on $\eta^{ \pm}$by the same scalar;

- the restriction of $\eta$ to $K_{M}=K \cap M$ lifts uniquely to a virtual representation of $K$. 
The Selberg zeta function for the pair $(\eta, \rho)$ is defined formally for $\sigma \in \mathbf{C}$ by

$$
Z_{\eta, \rho}(\sigma)=\exp \left(-\sum_{\substack{[\gamma] \in\left[\Gamma_{+}\right] \\ \gamma \sim e^{a} k^{-1} \in H}} \frac{\chi_{\text {orb }}\left(B_{[\gamma]} / \mathbb{S}^{1}\right)}{m_{[\gamma]}} \operatorname{Tr}[\rho(\gamma)] \frac{\operatorname{Tr}_{\mathrm{s}}^{E_{\eta}}\left[k^{-1}\right]}{\left.\left|\operatorname{det}\left(1-\operatorname{Ad}\left(e^{a} k^{-1}\right)\right)\right|_{\mathfrak{z}^{\perp}(\mathfrak{b})}\right|^{1 / 2}} e^{-\sigma \ell_{[\gamma]}}\right),
$$

where the sum is taken over the non elliptic conjugacy classes $[\gamma]$ of $\Gamma$ such that $\gamma$ can be conjugate by elements of $G$ into the Cartan subgroup $H$.

In [Sh18, Section 6], we have shown that the adjoint action of $K_{M}$ on $\mathfrak{p}_{\mathfrak{m}}$ lifts uniquely to a virtual representation of $K$. Let $\widehat{\eta}=\widehat{\eta}^{+}-\widehat{\eta}^{-}$be the unique virtual representation of $K$ such that

$$
\left.\widehat{\eta}\right|_{K_{M}}=\Lambda^{\bullet}\left(\mathfrak{p}_{\mathfrak{m}}^{*}\right) \widehat{\otimes}_{\mathbf{R}} \eta
$$

The Casimir operator of $\mathfrak{g}$ acts as a generalised Laplacian $C^{\mathfrak{g}, Z, \widehat{\eta}^{ \pm}, \rho}$ on the smooth sections over $Z$ of the locally homogenous vector bundle, induced by $\widehat{\eta}^{ \pm}$, twisted by the flat vector bundle $F$ (see (2.26)). By the general theory on elliptic differential operators, the regularised determinant $\operatorname{det}\left(C^{\mathfrak{g}, Z, \hat{\eta}^{ \pm}, \rho}+\sigma\right)$ is holomorphic on $\sigma \in \mathbf{C}$. Following [Sh18, Section 7], using Müller's Selberg trace formula for arbitrary twists [Mü11], in Section 5, we show that for $\operatorname{Re}(\sigma) \gg 1$, up to a multiplication by a non zero entire function, $Z_{\eta, \rho}(\sigma)$ is just the graded regularised determinant

$$
\frac{\operatorname{det}\left(C^{\mathfrak{g}, Z, \widehat{\eta}^{+}, \rho}+\sigma_{\eta}+\sigma^{2}\right)}{\operatorname{det}\left(C^{\mathfrak{g}, Z, \widehat{\eta}^{-}, \rho}+\sigma_{\eta}+\sigma^{2}\right)}
$$

where $\sigma_{\eta} \in \mathbf{R}$ is some constant. In this way, we deduce the meromorphic extension of $Z_{\eta, \rho}$ and we get precise information about the poles and zeros as well as their multiplicities.

In [Sh18, Section 6], we have shown that the adjoint representations $\eta_{j}$ of $M$ on $\Lambda^{j}\left(\mathfrak{n}^{*}\right) \otimes_{\mathbf{R}} \mathbf{C}$ satisfy our two previous assumptions. Using the fact that $R_{\rho}$ is an alternating product of $Z_{\eta_{j}, \rho}$, we deduce the meromorphic extension of $R_{\rho}$. In particular, up to a multiplication by a non zero entire function, $R_{\rho}$ is a product of the graded regularised determinants (0.25). Comparing this with the weighted regularised determinant of the flat Laplacian (0.5), we get (0.14).

As in [Sh18, Section 8], the proof of (0.15) requires a detailed analysis on the right regular representation of $G$ on the left $\Gamma$-invariant space $C^{\infty}\left(G, \mathbf{C}^{r}\right)^{\Gamma}$. In general, $C^{\infty}\left(G, \mathbf{C}^{r}\right)^{\Gamma}$ is not unitarisable. Let $\mathscr{Z}\left(\mathfrak{g}_{\mathbf{C}}\right)$ be the centre of the enveloping algebra of the complexified Lie algebra $\mathfrak{g}_{\mathbf{C}}$. Given a character $\chi: \mathscr{Z}\left(\mathfrak{g}_{\mathbf{C}}\right) \rightarrow \mathbf{C}$, let $V_{\chi}(\Gamma, \rho) \subset C^{\infty}\left(G, \mathbf{C}^{r}\right)^{\Gamma}$ be the subspace of $C^{\infty}\left(G, \mathbf{C}^{r}\right)^{\Gamma}$ consisting of the $K$-finite elements such that for all $z \in \mathscr{Z}\left(\mathfrak{g}_{\mathbf{C}}\right), z-\chi(z)$ acts nilpotently. In Section 6.2 , we will show that $V_{\chi}(\Gamma, \rho)$ is a Harish-Chandra $\left(\mathfrak{g}_{\mathbf{C}}, K\right)$-module with a generalised infinitesimal character $\chi$ (see Definitions 6.1 and 6.2). Proceeding similarly as in [Sh18, Section 8], we can deduce that $C_{\rho}$ and $r_{\rho}$ are determined by the Euler characteristics of certain cohomologies of $V_{\chi_{1}}(\Gamma, \rho)$, where $\chi_{1}$ is the trivial character. The proof of (0.15) reduces to showing $V_{\chi_{1}}(\Gamma, \rho)=0$.

When $\rho$ is unitary, using fundamental results of Vogan-Zuckerman [VoZu84], Vogan [Vo84], and Salamanca-Riba [SR99], in [Sh18, Proposition 8.12], we have shown that $V_{\chi_{1}}(\Gamma, \rho)=0$ if and only if $\rho$ is acyclic. When $\rho$ is not unitary, even if $\rho$ is acyclic, $V_{\chi_{1}}(\Gamma, \rho)$ does not always vanish (see [Mü20, (1.19)]). 
In Section 6 , using a perturbation argument, we will show that $V_{\chi_{\mathbf{1}}}(\Gamma, \rho)$ vanishes if $\rho$ is close enough to an acyclic and unitary representation $\rho_{0}$. Indeed, if $\left\{z_{i}\right\}_{i=1}^{\operatorname{dim}_{i=1}}$ is a system of generators $\mathscr{Z}\left(\mathfrak{g}_{\mathbf{C}}\right)$, we need to show that the operators $z_{i}-\chi_{\mathbf{1}}\left(z_{i}\right)$ act invertiblely on sections of certain locally homogenous vector bundle (see Proposition 6.7) twisted by the flat vector bundle associated to $\rho$. Note that $z_{i}-\chi_{\mathbf{1}}\left(z_{i}\right)$ are differential operators. If $\rho$ is close enough to $\rho_{0}$ in the sense of $C^{k}$ with $k$ big enough, all the above operators are lower order small perturbations of the corresponding operators associated to $\rho_{0}$ which are invertible. From the above considerations, we can deduce the desired invertibility.

Remark that in the case of hyperbolic manifolds, only the flat Laplacian is involved. Since the flat Laplacian is the square of the flat Hodge-Dirac operator, and since the flat Hodge-Dirac operator is a perturbation of an invertible self-adjoint Hodge-Dirac operator associated to $\rho_{0}$ by a small 0 -th order operator. Therefore, we can take $k=0$ (see [BrKa08, Proposition 6.8] and [Mü20, Lemma 6.2]).

In summary, the principle of the proofs is similar to [Sh18]. In effect, we refer to [Sh18] as much as necessary, and we only develop the proofs when the arguments of [Sh18] do not extend in a straightforward way. Let us remark finally that

- as in the case where $\rho$ is unitary, Bismut's geometric orbital integral formula plays an essential role in our proof of Theorem 0.1 . Here, we use such formulas inexplicitly, since all the involved orbital integrals have been evaluated in [Sh18] and [ShY17].

- it is crucial that the adjoint representations of $K_{M}$ on $\mathfrak{p}_{\mathfrak{m}}$ and $\mathfrak{n}$ lift to virtual representations of $K$. In [Sh18], we prove this fact by the classification theory of simple real Lie algebras (0.18). One of the contributions of the current paper is to give a simple new conceptual proof of a complexified version of [Sh18, Theorem 6.11], which is enough for our applications.

0.7. Organisation of the article. This article is organised as follows. In Section 1, we introduce the generalised Laplacian, its heat kernel, its regularised determinant, as well as the flat Laplacian and the Cappell-Miller analytic torsion.

In Section 2, we show the Selberg trace formula with arbitrary twists for the heat operator associated to the Casmir, which is originally due to Müller [Mü11].

In Section 3, we give a simple new conceptual proof for the lifting property [Sh18, Theorem 6.11].

In Section 4, we introduce the Ruelle zeta function $R_{\rho}$, and we state Theorem 0.1 as Theorem 4.3.

In Section 5, we introduce the Selberg zeta function $Z_{\eta, \rho}$. We show the meromorphic extensions of $Z_{\eta, \rho}$ and $R_{\rho}$, and we establish Theorem 0.1 i) ii).

In Section 6, we show certain cohomological formulas for $C_{\rho}$ and $r_{\rho}$. We prove Theorem 0.1 iii).

In Section 7, we extend Theorem 0.1 to orbifolds.

0.8. Notation. Throughout the paper, we use the superconnection formalism of [Q85] and [BeGeV04, Section 1.3]. If $A$ is a $\mathbf{Z}_{2}$-graded algebra and if $a, b \in A$, the supercommutator $[a, b]$ is given by

$$
a b-(-1)^{\operatorname{deg} a \operatorname{deg} b} b a .
$$


If $B$ is another $\mathbf{Z}_{2}$-graded algebra, we denote by $A \widehat{\otimes} B$ the super tensor product algebra of $A$ and $B$. If $E=E^{+} \oplus E^{-}$is a $\mathbf{Z}_{2}$-graded vector space, the algebra $\operatorname{End}(E)$ is $\mathbf{Z}_{2}$-graded. If $\tau= \pm 1$ on $E^{ \pm}$and if $a \in \operatorname{End}(E)$, the supertrace $\operatorname{Tr}_{\mathrm{s}}[a]$ is defined by $\operatorname{Tr}[\tau a]$.

If $V$ is a real vector space, we will use the notation $V_{\mathbf{C}}=V \otimes_{\mathbf{R}} \mathbf{C}$ for its complexification. If $M$ is a topological group, we will denote by $M^{0}$ the connected component of the identity in $M$. We make the convention that $\mathbf{N}=\{0,1,2, \ldots\}$, $\mathbf{N}^{*}=\{1,2, \ldots\}, \mathbf{R}_{+}^{*}=(0, \infty)$.

\section{THE GENERALised LAPLACIAN}

The purpose of this section is to recall some properties of a generalised Laplacian defined on a closed manifold. The generalised Laplacian $P$ is a second order (not necessarily self-adjoint) elliptic differential operator, whose principal symbol is a positive scalar. We show that the regularised determinant $\operatorname{det}(\sigma+P)$ is a holomorphic function on $\sigma \in \mathbf{C}$. Our proof of the meromorphic extension of the Ruelle zeta function associated to arbitrary twist given in Section 5 relies on this fact.

This section is organised as follows. In Sections 1.1-1.3, we introduce the generalised Laplacian, the associated semigroup, and the regularised determinant.

In Section 1.4, we introduce a flat vector bundle and its holonomy representation. We establish an estimate on the growth of the holonomy representation.

In Section 1.5, we construct a generalised Laplacian acting on the sections of a flat vector bundle. We relates its heat kernel with the lifted heat kernel on the universal cover.

Finally, in Section 1.6, given a flat vector bundle, we recall the construction of the flat Laplacian of Cappell-Miller, which is a generalised Laplacian. We introduce the complex valued analytic torsion of Cappell-Miller under the assumption that the flat Laplacian is invertible.

1.1. The spectral theory of a generalised Laplacian. Let $Z$ be a closed manifold of dimension $m$. Let $E$ be a complex vector bundle on $Z$. Let $C^{\infty}(Z, E)$ be the space of $C^{\infty}$-sections of $E$.

Let $g^{T Z}$ be a Riemannian metric on $Z$. Denote by $d v_{Z} \in \Omega^{m}(Z, o(T Z))$ the associated Riemannian volume form, where $o(T Z)$ is the orientation line bundle of $Z$. Let $g^{E}$ be a Hermitian metric on $E$. Let $\langle,\rangle_{g^{E}}$ be the induced Hermitian product on $E$. We equip $C^{\infty}(Z, E)$ with an $L^{2}$-product defined for $u_{1}, u_{2} \in C^{\infty}(Z, E)$ by

$$
\left\langle u_{1}, u_{2}\right\rangle_{L^{2}}=\int_{z \in Z}\left\langle u_{1}(z), u_{2}(z)\right\rangle_{g^{E}} d v_{Z} .
$$

Let $L^{2}(Z, E)$ be the space of $L^{2}$-sections of $E$. For $s \in \mathbf{R}$, let $\mathscr{H}^{s}(Z, E)$ be the $s$ Sobolev space of sections of $E$.

Let $P$ be a generalised Laplacian acting on $C^{\infty}(Z, E)$ in the sense of [BeGeV04, Definition 2.2]. Namely, if $|\cdot|_{g^{T Z, *}}^{2}$ is the dual metric on $T^{*} Z$ induced by $g^{T Z}$, then $P$ is a second order elliptic differential operator with the principal symbol

$$
\sigma(P)(x, \xi)=|\xi|_{g_{x}^{T Z, *}}^{2} \cdot \operatorname{id}_{E_{x}}, \quad \quad \text { for }(x, \xi) \in T^{*} Z .
$$


Equivalently, there is a metric connection $\nabla^{E}$ on $E$ and a first order differential operator $A$ such that

$$
P=\left(\nabla^{E}\right)^{*} \nabla^{E}+A
$$

where $\left(\nabla^{E}\right)^{*}: C^{\infty}\left(Z, T^{*} Z \otimes_{\mathbf{R}} E\right) \rightarrow C^{\infty}(Z, E)$ is the formal adjoint of $\nabla^{E}$ with respect to the $L^{2}$-product induced by $g^{E}$ and $g^{T Z}$.

By (1.3), there are $c>0$ and $C>0$ such that for any $u \in C^{\infty}(Z, E)$,

$$
\operatorname{Re}\langle P u, u\rangle_{L^{2}} \geqslant c\|u\|_{\mathscr{H}^{1}}^{2}-C\|u\|_{L^{2}}^{2} .
$$

For $s_{1}, s_{2} \in \mathbf{R}$, if $L$ is a bounded operator from $\mathscr{H}^{s_{1}}(Z, E)$ to $\mathscr{P}^{s_{2}}(Z, E)$, denoted by $\|L\|_{\mathscr{H}^{s_{1}}, \mathscr{P}^{s_{2}}}$ the corresponding operator norm. Consider $P$ as an operator with domain $\mathscr{H}^{2}(Z, E)$. Since $P$ is elliptic, the unbounded operator $\left(P, \mathscr{P}^{2}(Z, E)\right)$ is closed. By [S01, Theorem 9.3], $\left(P, \mathscr{H}^{2}(Z, E)\right)$ has a compact resolvent, so that its spectrum $\operatorname{Sp}(P)$ is discrete. Moreover, for any $\epsilon>0$, there is $r>0$ such that

$$
\operatorname{Sp}(P) \subset\{\lambda \in \mathbf{C}:|\lambda|<r \text { or }|\operatorname{Im}(\lambda)|<\epsilon \operatorname{Re}(\lambda)\} .
$$

Also, there are $C_{\epsilon, r}>0$ and $C_{\epsilon, r, n}>0$ for all $n \in \mathbf{Z}$ such that for all $\lambda \in \mathbf{C}$ with $|\lambda| \geqslant r$ and $|\operatorname{Im}(\lambda)| \geqslant \epsilon \operatorname{Re}(\lambda)$, we have

$$
\left\|(\lambda-P)^{-1}\right\|_{L^{2}, L^{2}} \leqslant \frac{C_{\epsilon, r}}{|\lambda|}, \quad \quad\left\|(\lambda-P)^{-1}\right\|_{\mathscr{H}^{n}, \mathcal{H}^{n+2}} \leqslant C_{\epsilon, r, n} .
$$

Take $\lambda \in \operatorname{Sp}(P)$. Let $V_{P}(\lambda) \subset L^{2}(Z, E)$ be the characteristic space of $P$ associated to the eigenvalue $\lambda$. For $N \gg 1$ large enough, we have

$$
V_{P}(\lambda)=\operatorname{ker}(\lambda-P)^{N} \text {. }
$$

Set

$$
m_{P}(\lambda)=\operatorname{dim} V_{P}(\lambda) \in \mathbf{N} .
$$

Since $P$ is elliptic, $V_{P}(\lambda) \subset C^{\infty}(Z, E)$. Since $P$ is a relatively compact perturbation of the self-adjoint operator $\left(\nabla^{E}\right)^{*} \nabla^{E}$, by the Keldysh theorem [GoKr69, Theorem 10.1], we have

$$
L^{2}(Z, E)=\overline{\oplus_{\lambda \in \mathrm{Sp}(P)} V_{P}(\lambda)}
$$

In particular, $\operatorname{Sp}(P)$ is not empty and is an infinite set.

1.2. The semigroup of a generalised Laplacian. By (1.4), (1.6), and by the Lumer-Phillips theorem [Yo95, p. 250], $-P$ is the generator of a $C^{0}$-semigroup $\exp (-t P)$ on $L^{2}(Z, E)$.

Fix $r>0$ and $\epsilon>0$ such that (1.5) and (1.6) hold. Let $\Gamma=\Gamma_{1} \cup \Gamma_{2} \cup \Gamma_{3}$ be the contour in the Figure 1.1 with the indicated orientation.

Proposition 1.1. For $t>0$, the following identity of bounded operators in $L^{2}(X, E)$ holds

$$
\exp (-t P)=\frac{1}{2 i \pi} \int_{\Gamma} e^{-t \lambda}(\lambda-P)^{-1} d \lambda
$$




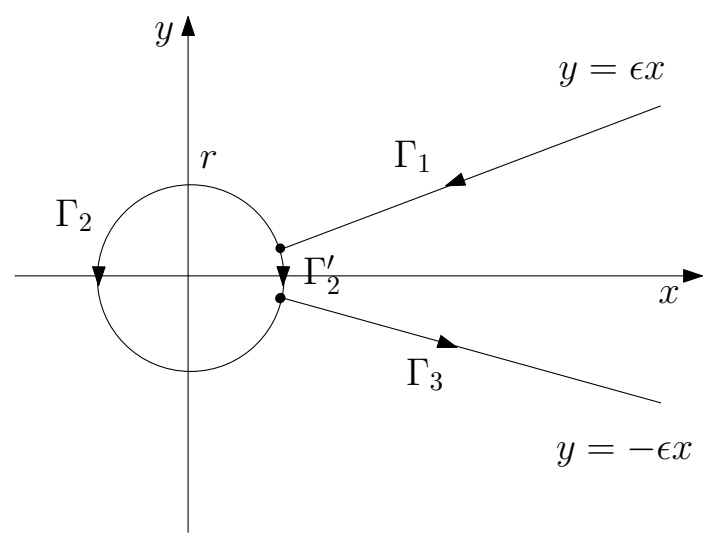

FIGURE 1.1. The contour.

The heat operator $\exp (-t P)$ has a smooth integral kernel. In particular, it is in trace class such that

$$
\operatorname{Tr}[\exp (-t P)]=\sum_{\lambda \in \operatorname{Sp}(P)} m_{P}(\lambda) e^{-t \lambda},
$$

where the right hand side of (1.11) converges absolutely.

Proof. By the first estimate of (1.6), the integral on the right-hand side of (1.10) converges absolutely in the space of bounded operators. By the general theory of $C^{0}$-semigroup [Pa83, Theorem I.7.7], we get (1.10).

Using integration by parts, we find that for any $k \in \mathbf{N}$, we have

$$
\exp (-t P)=\frac{(-1)^{k} k !}{2 i \pi t^{k}} \int_{\Gamma} e^{-t \lambda}(\lambda-P)^{-(k+1)} d \lambda .
$$

Take $k>m / 2$. The imbedding $\mathscr{H}^{2 k}(Z, E) \rightarrow L^{2}(Z, E)$ is in trace class. Denote by $\|\cdot\|_{\text {tr }}$ the trace norm. By the second estimate of (1.6), there is $C>0$ such that for all $\lambda \in \Gamma$, we have

$$
\left\|(\lambda-P)^{-(k+1)}\right\|_{\operatorname{tr}} \leqslant C .
$$

By (1.13), the right-hand side of (1.12) converges in the space of trace class operators. In particular, $\exp (-t P)$ is in trace class. Similar methods show that for any $s, s^{\prime} \in \mathbf{R}, \exp (-t P)$ maps $\mathscr{H}^{s}(Z, E)$ to $\mathscr{H}^{s^{\prime}}(Z, E)$ continuously. In particular, $\exp (-t P)$ has a smooth integral kernel.

We claim that

$$
\operatorname{Sp}(\exp (-t P))=\{0\} \cup\left\{e^{-t \lambda}: \lambda \in \operatorname{Sp}(P)\right\},
$$

and that each $e^{-t \lambda}$ has the multiplicity $m_{P}(\lambda)$. Indeed, it is clear that $e^{-t \lambda}$ with $\lambda \epsilon$ $\operatorname{Sp}(P)$ is the spectrum of $\exp (-t P)$. Since $\operatorname{Sp}(P)$ is infinite and since $\operatorname{Sp}(\exp (-t P))$ is a closed set, we see that $0 \in \mathrm{Sp}(\exp (-t P))$. Assume that $\mu \in \mathbf{C} \backslash\{0\}$ does not equal to any $e^{-t \lambda}$ with $\lambda \in \operatorname{Sp}(P)$. Note that $\exp (-t P)$ is compact. For $\delta>0$ small enough, the spectral projection of $\exp (-t P)$ to the characteristic space of $\mu$ is given by

$$
\Pi_{\mu}=\frac{1}{2 i \pi} \int_{|\lambda-\mu|=\delta} \frac{d \lambda}{\lambda-\exp (-t P)} .
$$


By our choice of $\mu, \Pi_{\mu}$ varnishes on $\oplus_{\lambda \in \operatorname{Sp}(P)} V_{P}(\lambda)$. By (1.9), $\Pi_{\mu}=0$, from which

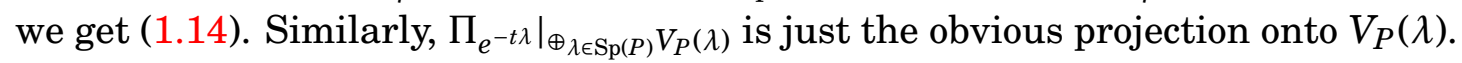
By (1.9), $\Pi_{e^{-t \lambda}}$ coincides with the spectral projection of $P$ onto $V_{P}(\lambda)$. Thus, the algebraic multiplicity of $\mu=e^{-t \lambda}$ is equal to $m_{P}(\lambda)$.

By (1.14) and by Lidskii's Theorem [GoKr69, Theorem 8.4], we get (1.11).

Let us study the long time exponential decay and the short time asymptotic of the heat trace $\operatorname{Tr}[\exp (-t P)]$. We choose $r>0$ and $\epsilon>0$ such that (1.5) and (1.6) hold and that

$$
\operatorname{Sp}(P) \cap\{z \in \mathbf{C}:|z|=r\}=\varnothing .
$$

Let $\pi_{<}$be the spectral projection of $P$ onto the space $\oplus_{\lambda \in \operatorname{Sp}(P):|\lambda|<r} V_{P}(\lambda)$. Then

$$
\pi_{<}=\frac{1}{2 i \pi} \int_{|\lambda|=r} \frac{d \lambda}{\lambda-P} .
$$

Set

$$
\pi_{>}=1-\pi_{<}
$$

Proposition 1.2. There are $c>0$ and $C>0$ such that for $t \geqslant 1$, we have

$$
\left\|\exp (-t P) \pi_{>}\right\|_{t r} \leqslant C e^{-c t} .
$$

Proof. Let $\Gamma^{\prime}=\Gamma_{1} \cup \Gamma_{2}^{\prime} \cup \Gamma_{3}$ be the oriented contour indication in the Figure 1.1. Clearly, there is $c>0$ such that if $\lambda \in \Gamma^{\prime}$ and $t>0$,

$$
\left|e^{-t \lambda}\right| \leqslant e^{-c t}
$$

By (1.9), (1.10), (1.12), (1.16)-(1.18), for $k \in \mathbf{N}$, we can deduce that

$$
\exp (-t P) \pi_{>}=\frac{(-1)^{k} k !}{2 i \pi t^{k}} \int_{\Gamma^{\prime}} e^{-t \lambda}(\lambda-P)^{-(k+1)} d \lambda
$$

Note that the integral converges for the operator norm $\|\cdot\|_{L^{2}, L^{2}}$.

If $k>m / 2$, by the resolvent identity, the maps $\lambda \in(\operatorname{Sp}(P))^{c} \rightarrow\left\|(\lambda-P)^{-(k+1)}\right\|_{\operatorname{tr}}$ is continuous. Since $\Gamma_{2}^{\prime}$ is compact, by (1.16), there is $C>0$ such that for all $\lambda \in \Gamma_{2}^{\prime}$, we have

$$
\left\|(\lambda-P)^{-(k+1)}\right\|_{\operatorname{tr}} \leqslant C .
$$

By (1.13), (1.20), and (1.22), if $k>m / 2$, the integral on the right-hand side of (1.21) converges for the trace norm and we get (1.19).

By [BeGeV04, Defintion 2.15, Lemma 2.34], the integral kernel of $\exp (-t P)$ is exactly the one constructed in [BeGeV04, Section 2.4]. As a consequence of [BeGeV04, Theorem 2.30], we have the following proposition.

Proposition 1.3. There are families of complex numbers $\left\{a_{k} \in \mathbf{C}\right\}_{k \in \mathbf{N}}$ and of positive real numbers $\left\{C_{k}>0\right\}_{k \in \mathbf{N}}$ such that for $k \in \mathbf{N}$ and $t \in(0,1]$, we have

$$
\left|\operatorname{Tr}[\exp (-t P)]-\frac{1}{t^{m / 2}} \sum_{i=0}^{k} a_{i} t^{i}\right| \leqslant C_{k} t^{k+1-m / 2} .
$$


1.3. The regularised determinant. Fix $r>0$ and $\epsilon>0$ such that (1.5), (1.6), and (1.16) hold. Set

$$
\operatorname{det}_{<}(P)=\prod_{\lambda \in \operatorname{Sp}(P):|\lambda|<r} \lambda^{m(\lambda)} \in \mathbf{C}, \quad \operatorname{det}_{<}^{*}(P)=\prod_{\lambda \in \operatorname{Sp}(P): 0<|\lambda|<r} \lambda^{m(\lambda)} \in \mathbf{C}^{*} .
$$

For $s>m / 2$, set

$$
\theta_{>}(s)=-\frac{1}{\Gamma(s)} \int_{0}^{\infty} \operatorname{Tr}\left[\exp (-t P) \pi_{>}\right] t^{s-1} d s .
$$

By Propositions 1.2 and 1.3, proceeding as [BeGeV04, Section 9.6], we see that $\theta_{>}(s)$ has a meromorphic extension to $s \in \mathbf{C}$, which is holomorphic at $s=0$. Set

$$
\operatorname{det}_{>}(P)=\exp \left(\frac{\partial}{\partial s} \theta_{>}(0)\right) \in \mathbf{C}^{*}
$$

Definition 1.4. Define

$$
\operatorname{det}(P)=\operatorname{det}_{<}(P) \cdot \operatorname{det}_{>}(P) \in \mathbf{C}, \quad \operatorname{det}^{*}(P)=\operatorname{det}_{<}^{*}(P) \cdot \operatorname{det}_{>}(P) \in \mathbf{C}^{*} .
$$

By (1.11), it is easy to see that this definition does not depend on the choice of $r>0$ or $\epsilon>0$.

Theorem 1.5. The function $\operatorname{det}(\sigma+P)$ is holomorphic on $\sigma \in \mathbf{C}$. Its zeros are located at $\sigma=-\lambda$ with multiplicity $m_{P}(\lambda)$, where $\lambda \in \operatorname{Sp}(P)$.

Proof. Since $\sigma+P$ is a generalised Laplacian, it is enough to show our theorem near $\sigma=0$. Recall that $r>0$ and $\epsilon>0$ are chosen such that (1.5), (1.6), and (1.16) hold. There is a small neighborhood $U \subset \mathbf{C}$ of 0 such that $U \cap\{-\operatorname{Sp}(P)\} \subset\{0\}$, and that (1.5), (1.6), and (1.16) still hold for all $\sigma+P$ with $\sigma \in U$.

We have the identity of functions on $U$,

$$
\operatorname{det}(\sigma+P)=\operatorname{det}_{<}(\sigma+P) \operatorname{det}_{>}(\sigma+P) .
$$

By our construction, $\operatorname{det}_{>}(\sigma+P)$ is a holomorphic function without zero on $U$, and that $\operatorname{det}_{<}(P+\sigma)$ is a polynomial function on $U$ where the only possibility of zeros is situated at $\sigma=0$ with multiplicity $m_{P}(0)$. The proof of our theorem is completed.

Remark 1.6. The statements of Propositions 1.1-1.3, and Theorem 1.5 hold true when $Z$ is a Riemannian closed orbifold and when $E$ is a Hermitian orbifold vector bundle on $Z$. Indeed, the proofs of Propositions 1.1 and 1.2 are based on (1.5) and (1.6), which are obtained using the peusdodifferential calculus. Using the peusdodifferential calculus on orbifolds [Ma05, p. 2213], the generalisations of (1.5) and (1.6) to orbifolds are straightforward. The proof of Proposition 1.3 in the orbifold setting is originally due to $\mathrm{Ma}$ [Ma05, Proposition 2.1]. The generalisation of Theorem 1.5 is a consequence of the orbifold version of Propositions 1.1-1.3.

1.4. Flat vector bundles. Let $Z$ be a connected closed manifold. Let $\Gamma=\pi_{1}(Z)$ be the fundamental group of $Z$. Let $\hat{\pi}: X \rightarrow Z$ be the universal cover of $Z$. The group $\Gamma$ acts on the left on $X$ as the deck transformation, so that

$$
Z=\Gamma \backslash X \text {. }
$$


Let $g^{T Z}$ be a Riemannian metric on $Z$. It lifts to a Riemannian metric $g^{T X}$ on $X$, so that $\Gamma$ acts on $X$ isometrically. Let $d_{X}(\cdot, \cdot)$ be the Riemannian distance on $X$. If $\gamma \in \Gamma$, let $d_{\gamma}: X \rightarrow \mathbf{R}$ be the displacement function on $\mathrm{X}$, i.e.,

$$
d_{\gamma}(x)=d_{X}(x, \gamma x)
$$

Set

$$
\ell_{\gamma}=\inf _{x \in X} d_{\gamma}(x)
$$

If $i_{Z}>0$ is the injectivity radius of $Z$, then for $\gamma \in \Gamma$ and $\gamma \neq 1$,

$$
\ell_{\gamma} \geqslant i_{Z}
$$

Let $F$ be a flat vector bundle of rank $r$ on $Z$ with flat connection $\nabla^{F}$. Let $\rho: \Gamma \rightarrow$ $\mathrm{GL}_{r}(\mathbf{C})$ be the holonomy representation of $F$, so that

$$
F=\Gamma \backslash\left(X \times \mathbf{C}^{r}\right) .
$$

Take $x \in X$ such that $\widehat{\pi}(x)=z$. Then $\rho(\gamma)$ can be considered as an element in $\operatorname{End}\left(F_{z}\right)$ defined by the parallel transport with respect to the flat connection $\nabla^{F}$ along the loop on $Z$ based at $z$, which is obtained by the projection of a path on $X$ from $\gamma x$ to $x$.

Let $g^{F}$ be a Hermitian metric on $F$.

Proposition 1.7. There is $C>0$ such that for any $\gamma \in \Gamma, x \in X$ with $z=\widehat{\pi}(x)$,

$$
|\rho(\gamma)|_{g_{z}^{F}} \leq C e^{C d_{\gamma}(x)}, \quad|\operatorname{Tr}[\rho(\gamma)]| \leq C e^{C \ell_{\gamma}}
$$

Proof. Following [BZ92, Definitions 4.1, 4.2], set

$$
\omega^{F}=\left(g^{F}\right)^{-1}\left(\nabla^{F} g^{F}\right) \in \Omega^{1}(Z, \operatorname{End}(F)),
$$

and

$$
\nabla^{F, u}=\nabla^{F}+\frac{1}{2} \omega^{F}
$$

Then, $\nabla^{F, u}$ is a connection on $F$ which preserves the metric $g^{F}$.

Let $\left(x_{t}\right)_{0 \leqslant t \leqslant t_{0}}$ be a smooth path on $X$ of speed 1 from $\gamma x$ to $x$. We trivialise $F$ over $\widehat{\pi}\left(x\right.$. ) by the parallel transport with respect to the metric connection $\nabla^{F, u}$. For $s, t \in\left[0, t_{0}\right]$, let $\tau_{t}^{s} \in \operatorname{Hom}\left(F_{\widehat{\pi} x_{s}}, F_{\widehat{\pi} x_{t}}\right)$ be the corresponding parallel transport. Let $U_{t} \in C^{\infty}\left(\left[0, t_{0}\right], \operatorname{End}\left(F_{z}\right)\right)$ be the unique solution of the differential equation

$$
\dot{U}_{t}-\frac{1}{2} \tau_{0}^{t} \omega_{\widehat{\pi} x_{t}}^{F}\left(\dot{x}_{t}\right) \tau_{t}^{0} U_{t}=0, \quad \quad U_{0}=\mathrm{Id} .
$$

As elements in $\operatorname{End}\left(F_{z}\right)$, we have

$$
\rho(\gamma)=\tau_{t_{0}}^{0} U_{t_{0}}
$$

Since $Z$ is compact, the smooth section $\omega^{F}$ is uniformly bounded. Since $\left|\dot{x}_{s}\right|=1$ and since $\tau_{t}^{s}$ preserves $g^{F}$, by Gronwall's inequality, there is $C>0$ independent of $x$, of $\gamma$, and of the path $x$, such that for all $t>0$,

$$
\left|U_{t}\right|_{g_{z}^{F}} \leqslant C e^{C t} \text {. }
$$

By (1.38) and (1.39), using again that $\tau_{t_{0}}^{0}$ is unitary with respect to $g_{z}^{F}$, we get

$$
|\rho(\gamma)|_{g_{z}^{F}} \leq C e^{C t_{0}}
$$


Since $t_{0}$ is the length of the path $\left(x_{t}\right)_{t \in\left[0, t_{0}\right]}$, by taking the infimum of all such paths, we get the first inequality of (1.34).

Using the trivial inequality on matrices, by the first inequality of (1.34), we have

$$
|\operatorname{Tr}[\rho(\gamma)]| \leqslant r|\rho(\gamma)|_{g_{z}^{F}} \leqslant r C e^{C d_{\gamma}(x)} .
$$

Since the constants $C$ and $r$ do not depend on $x$, by taking the infimum on $x \in X$ in (1.41), using (1.31), we get the second inequality of (1.34).

1.5. A generalised Laplacian and flat vector bundles. We use the notation of Section 1.4. Let $\widetilde{E}$ be a Hermitian vector bundle on $X$ with a metric connection $\nabla^{\widetilde{E}}$. Assume that the $\Gamma$-action on $X$ lifts to $\widetilde{E}$ and preserves the Hermitian metric and the connection $\nabla^{\widetilde{E}}$. Then $\left(\widetilde{E}, \nabla^{\widetilde{E}}\right)$ descends to a Hermitian vector bundle $E$ on $Z$ with a metric connection $\nabla^{E}$.

Let $P^{X}$ be a $\Gamma$-invariant self-adjoint generalised Laplacian acting on $C^{\infty}(X, \widetilde{E})$. Assume that

$$
P^{X}=\left(\nabla^{\widetilde{E}}\right)^{*} \nabla^{\widetilde{E}}+\widetilde{A}
$$

where $\widetilde{A} \in C^{\infty}(X, \operatorname{End}(\widetilde{E}))^{\Gamma}$. Since the Riemannian manifold $\left(X, g^{T X}\right)$ is complete, for $t>0$, it is classical that the heat operator $\exp \left(-t P^{X}\right)$ exists and has a smooth integral kernel. Let $p_{t}^{X}(x, y)$ be the smooth integral kernel with respect to the Riemannian volume $d v_{X}$.

Recall that $F$ is a flat vector bundle on $Z$ with holonomy $\rho: \Gamma \rightarrow \mathrm{GL}_{r}(\mathbf{C})$. We have the identification

$$
C^{\infty}(Z, E \otimes F)=\left(C^{\infty}(X, \widetilde{E}) \otimes \mathbf{C}^{r}\right)^{\Gamma}
$$

The operator $P^{X} \otimes$ id preserves the above $\Gamma$-invariant space. It descends to a generalised Laplacian $P^{Z, \rho}$ acting on $C^{\infty}(Z, E \otimes F)$. Since $\rho$ is not necessarily unitary, there is no obvious metric on $F$ such that $P^{Z, \rho}$ is self-adjoint. Still, by Proposition 1.1, the heat operator $\exp \left(-t P^{Z, \rho}\right)$ exists and has a smooth integral kernel. For $t>0$, the smooth integral kernel with respect to the Riemannian volume $d v_{Z}$ can be identified with a $\Gamma \times \Gamma$-invariant section $\widetilde{q}_{t}^{Z, \rho}(x, y)$ in $C^{\infty}(X \times$ $\left.X,\left(\widetilde{E} \otimes \mathbf{C}^{r}\right) \otimes\left(\widetilde{E} \otimes \mathbf{C}^{r}\right)^{*}\right)$.

Note that $p_{t}^{X}\left(\gamma^{-1} x, y\right) \in \operatorname{Hom}\left(\widetilde{E}_{y}, \widetilde{E}_{\gamma^{-1} x}\right)$. Let $\gamma_{*} \in \operatorname{Hom}\left(\widetilde{E}_{\gamma^{-1} x}, \widetilde{E}_{x}\right)$ be the obvious element. Then, $\gamma_{*} p_{t}^{X}\left(\gamma^{-1} x, y\right) \in \operatorname{Hom}\left(\widetilde{E}_{y}, \widetilde{E}_{x}\right)$. Recall that we have fixed a metric $g^{F}$ on $F$.

Theorem 1.8. Given $t_{0}>0, T_{0}>0$ with $t_{0}<T_{0}$ and given a bounded open set $X_{0} \subset X$, for any $s \in \mathbf{N}$, the sum on the right-hand side of (1.44) converges absolutely in the space of $C^{s}\left(\left[t_{0}, T_{0}\right] \times X_{0} \times X_{0}, \mathbf{C} \otimes\left(\widetilde{E} \otimes \mathbf{C}^{r}\right) \otimes\left(\widetilde{E} \otimes \mathbf{C}^{r}\right)^{*}\right)$, so that

$$
\widetilde{q}_{t}^{Z}(x, y)=\sum_{\gamma \in \Gamma} \rho(\gamma) \otimes \gamma_{*} p_{t}^{X}\left(\gamma^{-1} x, y\right) .
$$

Proof. We will first show that sum on the right hand side of (1.44) converges to a $\Gamma \times \Gamma$-invariant smooth section $q_{t}(x, y)$ and then show that $q_{t}(x, y)$ satisfies the heat equation. 
We fix $x_{0} \in X_{0}$. We claim that for any $\delta>0$, we have

$$
\sum_{\gamma \in \Gamma} \exp \left(-\delta d_{\gamma}^{2}\left(x_{0}\right)\right)<+\infty
$$

Indeed, by [Mi68b, Remark p. 1, Lemma 2] or [MaMar15, (3.19)], there is $C>0$ such that for all $r \geqslant 0$,

$$
\left|\left\{\gamma \in \Gamma: d_{\gamma}\left(x_{0}\right) \leqslant r\right\}\right| \leqslant C e^{C r} .
$$

For $\delta>0$, by (1.46), we have

$$
\begin{aligned}
\sum_{\gamma \in \Gamma} \exp \left(-\delta d_{\gamma}^{2}\left(x_{0}\right)\right)=\sum_{\gamma \in \Gamma} \int_{\delta d_{\gamma}^{2}\left(x_{0}\right)}^{\infty} \exp (-t) d t \\
=\int_{0}^{\infty} \exp (-t)\left|\left\{\gamma \in \Gamma: d_{\gamma}\left(x_{0}\right) \leqslant \sqrt{t / \delta}\right\}\right| d t \leqslant C \int_{0}^{\infty} e^{-t+C \sqrt{t / \delta}} d t<+\infty
\end{aligned}
$$

Since $X_{0}$ is bounded, by (1.34) and by triangle inequality, there is $C>0$ such that, for any $x \in X_{0}$, we have

$$
|\rho(\gamma)|_{g_{\tilde{\pi} x}^{F}} \leqslant C e^{C d_{\gamma}\left(x_{0}\right)}
$$

By finite propagation speed of solutions of hyperbolic equations [MaMar07, Theorem D.2.1], as in [MaMar15, Theorem 4], there are $c>0, C>0$ such that for any $t \in\left[t_{0}, T_{0}\right]$ and $x, y \in X$,

$$
\left|p_{t}^{X}(x, y)\right| \leqslant C \exp \left(-c d_{X}^{2}(x, y)\right) .
$$

Moreover, similar estimates hold uniformly on $\left[t_{0}, T_{0}\right] \times X \times X$, for all derivations on $t, x, y$. By (1.49) and by triangle inequality, there are $c>0, C>0$ such that for $t \in\left[t_{0}, T_{0}\right], x, y \in X_{0}, \gamma \in \Gamma$, we have

$$
\left|p_{t}^{X}\left(\gamma^{-1} x, y\right)\right| \leqslant C \exp \left(-c d_{\gamma}^{2}\left(x_{0}\right)\right)
$$

and similar estimates for all derivations on $t, x, y$.

By (1.45), (1.48), and (1.50), for all $s \in \mathbf{N}$, the sum on the right-hand side of (1.44) converges absolutely in $C^{s}\left(\left[t_{0}, T_{0}\right] \times X_{0} \times X_{0}, \mathbf{C} \otimes\left(\widetilde{E} \otimes \mathbf{C}^{r}\right) \otimes\left(\widetilde{E} \otimes \mathbf{C}^{r}\right)^{*}\right)$ to a $\Gamma \times \Gamma$-invariant smooth section $q_{t}(x, y)$, so that

$$
\begin{aligned}
\frac{\partial}{\partial t} q_{t}(x, y) & =\sum_{\gamma \in \Gamma} \rho(\gamma) \otimes \frac{\partial}{\partial t} \gamma_{*} p_{t}^{X}\left(\gamma^{-1} x, y\right), \\
P_{x}^{X} q_{t}(x, y) & =\sum_{\gamma \in \Gamma} \rho(\gamma) \otimes P_{x}^{X} \gamma_{*} p_{t}^{X}\left(\gamma^{-1} x, y\right),
\end{aligned}
$$

where $P_{x}^{X}$ denotes the differential operator $P^{X}$ acting on the variable $x$. By (1.51), using $\frac{\partial}{\partial t} p_{t}^{X}(x, y)=-P_{x}^{X} p_{t}^{X}(x, y)$, and using the fact that $p_{t}^{X}$ is $\Gamma \times \Gamma$-invariant, we get

$$
\frac{\partial}{\partial t} q_{t}(x, y)=-P_{x}^{X} q_{t}(x, y)
$$

Take $u \in C^{\infty}(Z, E \otimes F)$. We identify $u$ with its $\Gamma$-invariant lifting $\widetilde{u} \in\left(C^{\infty}(X, \widetilde{E}) \otimes \mathbf{C}^{r}\right)^{\Gamma}$. Let $F_{Z} \subset X$ be a fundamental domain ${ }^{2}$ of $Z$ in $X$. By (1.34), there is $C>0$, for

\footnotetext{
${ }^{2}$ For example, we can take $F_{Z}=\left\{x \in X\right.$ : for all $\left.\gamma \in \Gamma, d_{X}\left(x, x_{0}\right)<d_{X}\left(x, \gamma x_{0}\right)\right\}$.
} 
$y \in F_{Z}$ and $\gamma \in \Gamma$, we have

$$
|\widetilde{u}(\gamma y)| \leqslant C e^{C d_{\gamma}(y)} .
$$

By (1.45), (1.50), and (1.53), using Fubini's theorem, we have

$$
\int_{y \in F_{Z}} q_{t}(x, y) \widetilde{u}(y) d v_{X}=\int_{y \in X} p_{t}^{X}(x, y) \widetilde{u}(y) d v_{X} .
$$

By [BeGeV04, Definition 2.15, Lemma 2.34], it remains to show that for any $u \in$ $C^{\infty}(Z, E \otimes F)$, as $t \rightarrow 0$, we have

$$
\sup _{x \in F_{Z}}\left|\int_{y \in X} p_{t}^{X}(x, y) \widetilde{u}(y) d v_{X}-\widetilde{u}(x)\right| \rightarrow 0 .
$$

Let $F_{Z}^{0}, F_{Z}^{1}$ be two bounded open neighbourhoods of $\bar{F}_{Z}$ such that $\bar{F}_{Z}^{0} \subset F_{Z}^{1}$. Write

$$
\widetilde{u}=\widetilde{u}_{1}+\widetilde{u}_{2},
$$

where $\widetilde{u}_{1}$ and $\widetilde{u}_{2}$ are $C^{\infty}$-sections on $X$ such that $\operatorname{Supp}\left(\widetilde{u}_{1}\right) \subset F_{Z}^{1}$ and $\operatorname{Supp}\left(\widetilde{u}_{2}\right) \subset$ $X \backslash F_{Z}^{0}$. Since $\widetilde{u}_{1}$ has a compact support in $X$, by the Sobolev imbedding theorem, for $k>m / 4$, there is $C_{k}>0$ such that

$$
\begin{array}{r}
\left\|\exp \left(-t P^{X}\right) \widetilde{u}_{1}-\widetilde{u}_{1}\right\|_{C^{0}\left(F_{Z}^{1}\right)} \leqslant C_{k}\left\|\left(1+P^{X}\right)^{k}\left(\exp \left(-t P^{X}\right) \tilde{u}_{1}-\widetilde{u}_{1}\right)\right\|_{L^{2}(X)} \\
=C_{k}\left\|\exp \left(-t P^{X}\right)\left(1+P^{X}\right)^{k} \widetilde{u}_{1}-\left(1+P^{X}\right)^{k} \widetilde{u}_{1}\right\|_{L^{2}(X)} .
\end{array}
$$

Since $\left(1+P^{X}\right)^{k} \widetilde{u}_{1}$ is $L^{2}$ on $X$, by a property of the semigroup $\exp \left(-t P^{X}\right)$ and by (1.57), as $t \rightarrow 0$, we have

$$
\sup _{x \in F_{Z}}\left|\int_{y \in X} p_{t}^{X}(x, y) \widetilde{u}_{1}(y) d v_{X}-\widetilde{u}_{1}(x)\right| \rightarrow 0 .
$$

By finite propagation speed of solutions of hyperbolic equations [MaMar07, Theorem D.2.1], there are $c>0, C>0$, for $t \in(0,1], x, y \in X$,

$$
\left|p_{t}^{X}(x, y)\right| \leqslant \frac{C}{t^{m / 2}} \exp \left(-c \frac{d_{X}^{2}(x, y)}{t}\right) .
$$

Since $\operatorname{Supp}\left(\widetilde{u}_{2}\right) \subset X \backslash F_{Z}^{0}$ and since $d_{X}\left(X \backslash F_{Z}^{0}, F_{Z}\right)>c^{\prime}>0$, using (1.45), (1.53), and (1.59), we can deduce that there are $c>0$ and $C>0$, for $t \in(0,1]$,

$$
\sup _{x \in F_{Z}}\left|\int_{y \in X} p_{t}^{X}(x, y) \widetilde{u}_{2}(y) d v_{X}\right| \leqslant C e^{-c / t} .
$$

By (1.58) and (1.60), we get (1.55). The proof of our theorem is completed.

Corollary 1.9. For $t>0$, the right-hand side of the following identity convergences absolutely, so that

$$
\operatorname{Tr}\left[\exp \left(-t P^{Z, \rho}\right)\right]=\sum_{\gamma \in \Gamma} \operatorname{Tr}[\rho(\gamma)] \int_{x \in F_{Z}} \operatorname{Tr}^{\widetilde{E}}\left[\gamma_{*} p_{t}^{X}\left(\gamma^{-1} x, x\right)\right] d v_{X}
$$

Proof. This is a consequence of [MaMar07, Theorem D.1.5], Proposition 1.7, Theorem 1.8, and the estimates (1.34), (1.45), (1.50).

Set $\Gamma_{+}=\Gamma-\{\mathrm{id}\}$. We have a generalisation of [Sh18, Proposition 4.8]. 
Proposition 1.10. There are $c>0, C>0$ such that for any $x \in X$ and $t>0$,

$$
\sum_{\gamma \in \Gamma_{+}}|\operatorname{Tr}[\rho(\gamma)]|\left|p_{t}^{X}\left(\gamma^{-1} x, x\right)\right| \leqslant C \exp \left(-\frac{c}{t}+C t\right) .
$$

Proof. Since $X$ is a cover of a compact manifold, by [Sh18, (4-27)] or [MaMar15, Theorem 4]), there exist $c>0, C>0$ such that for $t>0, x, x^{\prime} \in X$, we have

$$
\left|p_{t}^{X}\left(x, x^{\prime}\right)\right| \leqslant \frac{C}{t^{m / 2}} \exp \left(-c \frac{d_{\gamma}^{2}(x)}{t}+C t\right) .
$$

By (1.32) and (1.63), there are $c_{1}>0, c_{2}>0$, and $C>0$ such that for $t>0, x \in X$, and $\gamma \in \Gamma_{+}$, we have

$$
\left|p_{t}^{X}\left(\gamma^{-1} x, x\right)\right| \leqslant C \exp \left(-\frac{c_{1}}{t}-c_{2} \frac{d_{\gamma}^{2}(x)}{t}+C t\right) .
$$

By (1.34) and (1.64), using $C d_{\gamma}(x) \leqslant c_{2} \frac{d_{\gamma}^{2}(x)}{2 t}+\frac{C^{2}}{2 c_{2}} t$, we get

$$
|\operatorname{Tr}[\rho(\gamma)]|\left|p_{t}^{X, \tau}\left(\gamma^{-1} x, x\right)\right| \leqslant C \exp \left(-\frac{c_{1}}{t}-c_{2} \frac{d_{\gamma}^{2}(x)}{2 t}+C^{\prime} t\right) .
$$

Now proceeding as in [Sh18, (4-31)], we get (1.62).

1.6. Flat Laplacian and the Cappell-Miller analytic torsion. Let $Z$ be a compact manifold of dimension $m$. Let $\left(F, \nabla^{F}\right)$ be a complex flat vector bundle on $Z$ with holonomy $\rho: \Gamma \rightarrow \mathrm{GL}_{r}(\mathbf{C})$. Let $\Omega^{\bullet}(Z, F)$ be the space of smooth differential forms with coefficients in $F$. Let $\left(\Omega^{\bullet}(Z, F), d^{Z}\right)$ be the de Rham complex, and let $H^{\bullet}(Z, F)$ be the corresponding de Rham cohomology.

Let $g^{T Z}$ be a Riemannian metric on $Z$. It induces a Riemannian metric $g^{T X}$ on $X$. Let $\langle,\rangle_{L^{2}}$ be the $L^{2}$-product on $\Omega^{\cdot}(X)$ induced by $g^{T X}$. Let $d^{X, *}$ be the formal adjoint of $d^{X}$. Set

$$
\square^{X}=\left[d^{X}, d^{X, *}\right]
$$

Then, $\square^{X}$ is a self-adjoint generalised Laplacian acting on $\Omega^{\bullet}(X)$.

We use the convention in Section 1.5 with $E=\Lambda^{\bullet}\left(T^{*} Z\right)$ and $\widetilde{E}=\Lambda^{\bullet}\left(T^{*} X\right)$. By (1.43), $d^{X} \otimes \mathrm{id}$ descends to the de Rham operator $d^{Z}$. Similarly, $d^{X, *} \otimes \mathrm{id}, \square^{X} \otimes \mathrm{id}$ descend to operators $d^{Z, *}, \square^{Z}$, so that

$$
\square^{Z}=\left[d^{Z}, d^{Z, *}\right] .
$$

The operator $\square^{Z}$ is a generalised Laplacian which will be called the flat Laplacian.

Let $\Omega_{0}^{\cdot}(Z, F)$ be the characteristic space of $\square^{Z}$ associated with the eigenvalue 0 . Since $d^{Z}$ commutes with $\square^{Z}$, we see that $\left(\Omega_{0}^{\cdot}(Z, F), d^{Z}\right)$ is a complex. By [CMil10, p. 181],

$$
H^{\bullet}\left(\Omega_{0}^{\bullet}(Z, F), d^{Z}\right) \simeq H^{\bullet}(Z, F)
$$

Set

$$
\chi_{\mathrm{CM}}^{\prime}(F)=\sum_{i=1}^{m}(-1)^{i} i \operatorname{dim} \Omega_{0}^{i}(Z, F) .
$$


Let $T_{\rho}(\sigma)$ be a meromorphic function on $\mathbf{C}$ defined by

$$
T_{\rho}(\sigma)=\prod_{i=1}^{m} \operatorname{det}\left(\sigma+\left.\square^{Z}\right|_{\Omega^{i}(Z, F)}\right)^{(-1)^{i} i} .
$$

As $\sigma \rightarrow 0$, we have

$$
T_{\rho}(\sigma)=\left\{\prod_{i=1}^{m} \operatorname{det}^{*}\left(\left.\square^{Z}\right|_{\Omega^{i}(Z, F)}\right)^{(-1)^{i} i}\right\} \sigma^{\chi_{\mathrm{CM}}^{\prime}(Z, F)}+\mathscr{O}\left(\sigma^{\chi_{\mathrm{CM}}^{\prime}(Z, F)+1}\right) .
$$

Definition 1.11. If $\square^{Z}$ is invertible, the complex valued analytic torsion of CappellMiller is defined by

$$
T_{\mathrm{CM}}(F)=\prod_{i=1}^{m} \operatorname{det}^{*}\left(\left.\square^{Z}\right|_{\Omega^{i}(Z, F)}\right)^{(-1)^{i} i} \in \mathbf{C}^{*} .
$$

We refer the reader to [CMil10] for the definition of the Cappell-Miller torsion in the case where $\square^{Z}$ is not invertible.

Assume that $\square^{Z}$ is invertible. By [CMil10, p. 179], if $Z$ is orientable and has even dimension, then $T_{\mathrm{CM}}(F)=1$. By [CMil10, Theorem 8.3], if $\operatorname{dim} Z$ is odd, $T_{\mathrm{CM}}(F)$ does not depend on the metric $g^{T Z}$. It becomes a topological invariant.

Let $\operatorname{Rep}\left(\Gamma, \mathbf{C}^{r}\right)$ be the set of all $r$-dimensional complex representations of $\Gamma$. It is well known that $\operatorname{Rep}\left(\Gamma, \mathbf{C}^{r}\right)$ has a natural structure of a complex algebraic variety (see [BrKa08, Section 13.6]). Let us follow [GolM88, Proposition 4.5] and [Mü20, Section 6.2]. Let $U \subset \operatorname{Rep}\left(\Gamma, \mathbf{C}^{r}\right)$ be a contractible neighbourhood of $\rho_{0}$. Consider the $\Gamma$-action on $U \times X \times \mathbf{C}^{r}$ defined by

$$
\gamma \cdot(\rho, x, v)=(\rho, \gamma x, \rho(\gamma) v)
$$

The projection on the quotient space

$$
\Gamma \backslash\left(U \times X \times \mathbf{C}^{r}\right) \rightarrow U \times Z
$$

define a vector bundle on $U \times Z$, whose restriction to $\{\rho\} \times Z$ is just the flat vector bundle with holonomy $\rho$. We write $F_{\rho}$ to emphasise the dependence on $\rho$.

Since $U$ is contractible, we have an identification of vector bundles over $U \times Z$,

$$
\Gamma \backslash\left(U \times X \times \mathbf{C}^{r}\right) \simeq U \times F_{\rho_{0}} .
$$

Note that the identification is non canonical and is only continuous in the variables in $U$.

For $\rho \in U$, by (1.75), we have a bundle isomorphism

$$
F_{\rho} \simeq F_{\rho_{0}} .
$$

Under this identification, the flat connection on $F_{\rho}$ can be written as

$$
\nabla^{F_{\rho}}=\nabla^{F_{\rho_{0}}}+A_{\rho}
$$

with $A_{\rho} \in \Omega^{1}\left(Z, \operatorname{End}\left(F_{\rho_{0}}\right)\right)$. For $k \in \mathbf{N}$ and $\epsilon>0$, we call $\rho$ is $C^{k} \epsilon$-close to $\rho_{0}$, if

$$
\left\|A_{\rho}\right\|_{C^{k}} \leqslant \epsilon \text {. }
$$

Recall that we have fixed a Riemannian metric on $T Z$. 
Proposition 1.12. If $\rho_{0} \in \operatorname{Rep}\left(\Gamma, \mathbf{C}^{r}\right)$ is unitary and acyclic, then there is $\epsilon>0$ such that if $\rho$ is $C^{0} \epsilon$-close to $\rho_{0}$, then the flat Laplacian on $F_{\rho}$ is invertible, so that

$$
T_{\mathrm{CM}}\left(F_{\rho}\right)=\prod_{i=1}^{m} \operatorname{det}^{*}\left(\left.\square^{Z}\right|_{\Omega^{i}\left(Z, F_{\rho}\right)}\right)^{(-1)^{i} i} \in \mathbf{C}^{*} .
$$

Proof. This is [Mü20, Lemma 6.2] ([BrKa08, Proposition 6.8]), whose proof is based on the invertibility of the first order elliptic differential operator $d^{Z}+d^{Z \text {,* }}$ (c.f. Proposition 6.7).

Remark 1.13. Assume that $Z$ is a closed orbifold and that $F$ is a flat orbifold vector bundle on $Z$. Given a Riemannian metric on $Z$, we can define the flat Laplacian, the regularised determinant, Cappell-Miller analytic torsion in the same way, so that (1.71) and Proposition 1.12 still hold true.

\section{MÜlleR's Selberg TRACE FORMUla}

The purpose of this section is to establish the Selberg trace formula for the heat operator of the Casimir on the locally symmetric space twisted by an arbitrary flat vector bundle. Such a formula is obtained by Müller [Mü11] for the functional of the Casimir with respect to an even Paley-Wiener function. The extension to the heat operator does not contain particular difficulties. We include some detail for completeness.

This section is organised as follows. In Sections 2.1-2.3, we introduce the real reductive group $G$, its maximal compact subgroup $K$, and the Casimir $C^{\mathfrak{g}}$, the associated symmetric space $X=G / K$, and the $K$-principal bundle $p: G \rightarrow X$. Given a finite dimensional unitary representation $\tau: K \rightarrow U\left(E_{\tau}\right)$ of $K$, we construct the associated Hermitian vector bundle $\mathscr{E}_{\tau}$ on $X$. The Casimir operator on $C^{\infty}\left(X, \mathscr{E}_{\tau}\right)$ is a self-adjoint generalised Laplacian $C^{\mathfrak{g}, X, \tau}$.

In Sections 2.4 and 2.5, we introduce the semisimple elements in $G$ and the associated semisimple orbital integrals with respect to the heat operator of $C^{\mathfrak{g}, X, \tau}$.

Finally, in Section 2.6, we introduce a discrete cocompact subgroup $\Gamma \subset G$ of $G$ and the corresponding locally symmetric space $\Gamma \backslash X$. Given a finite dimensional representation of $\Gamma$, the Casimir operator descends to a generalised Laplacian on $Z$. We establish the Selberg trace formula for the associated heat operator.

2.1. Real reductive groups. Let $G$ be a linear connected real reductive group [K86, p. 3], and let $\theta \in \operatorname{Aut}(G)$ be the Cartan involution. That means $G$ is a closed connected group of real matrices that is stable under transpose, and $\theta$ is the composition of transpose and inverse of matrices. Let $K \subset G$ be the subgroup of $G$ fixed by $\theta$, so that $K$ is a maximal compact subgroup of $G$.

Let $\mathfrak{g}, \mathfrak{k}$ be the Lie algebras of $G, K$. The Cartan involution acts by differential as Lie algebra automorphism on $\mathfrak{g}$, which will still be denoted by $\theta$. Then $\mathfrak{k}$ is the eigenspace of $\theta$ associated with the eigenvalue 1 . Let $\mathfrak{p}$ be the eigenspace of $\theta$ associated with the eigenvalue -1 , so that

$$
\mathfrak{g}=\mathfrak{p} \oplus \mathfrak{k} .
$$

Set

$$
m=\operatorname{dim} \mathfrak{p}, \quad n=\operatorname{dim} \mathfrak{k} .
$$


By [K86, Proposition 1.2], we have the diffeomorphism

$$
(Y, k) \in \mathfrak{p} \times K \rightarrow e^{Y} k \in G .
$$

Let $B$ be a real-valued nondegenerate bilinear symmetric form on $\mathfrak{g}$ which is invariant under the adjoint action $\operatorname{Ad}$ of $G$, and also under $\theta$. Then (2.1) is an orthogonal splitting of $\mathfrak{g}$ with respect to $B$. We assume $B$ to be positive-definite on $\mathfrak{p}$, and negative-definite on $\mathfrak{k}$. The form $\langle\cdot, \cdot\rangle=-B(\cdot, \theta \cdot)$ defines an $\operatorname{Ad}(K)$-invariant scalar product on $\mathfrak{g}$ such that the splitting (2.1) is still orthogonal. We denote by $|\cdot|$ the corresponding norm.

Let $Z_{G} \subset G$ be the centre of $G$ with Lie algebra $\mathfrak{z} \mathfrak{g} \subset \mathfrak{g}$. By [K86, Corollary 1.3], $Z_{G}$ is a (possibly non connected) reductive group with maximal compact subgroup $Z_{G} \cap K$ with the Cartan decomposition

$$
\mathfrak{z}_{\mathfrak{g}}=\mathfrak{z}_{\mathfrak{p}} \oplus \mathfrak{z} \mathfrak{k}
$$

Since $\mathfrak{z}_{\mathfrak{p}}$ commutes with $Z_{G} \cap K$, by (2.3), we have an identification of the groups

$$
Z_{G}=\exp \left(\mathfrak{z}_{\mathfrak{p}}\right) \times\left(Z_{G} \cap K\right) .
$$

Let $G_{\mathrm{ss}} \subset G$ be the connected subgroup of $G$ associated with the Lie algebra $[\mathfrak{g}, \mathfrak{g}]$. By [K02, Corollary 7.11], $G_{\mathrm{ss}}$ is a closed subgroup of $G$. Moreover, $G_{\mathrm{ss}}$ is semisimple and

$$
G=G_{\mathrm{ss}} \cdot Z_{G}^{0}
$$

Proposition 2.1. If $G$ has a compact center, any one dimensional real representation of $G$ is trivial.

Proof. This is a consequence of (2.6) and of the fact that any morphism of groups from a connected semisimple Lie group or a connected compact Lie group to $\mathbf{R}^{*}$ is trivial.

Let $\mathfrak{g}_{\mathbf{C}}=\mathfrak{g} \otimes_{\mathbf{R}} \mathbf{C}$ be the complexification of $\mathfrak{g}$ and let $\mathfrak{u}=\sqrt{-1} \mathfrak{p} \oplus \mathfrak{k}$ be the compact form of $\mathfrak{g}$. By $\mathbf{C}$-linearity, the bilinear form $B$ extends to a complex symmetric bilinear form on $\mathfrak{g}_{\mathbf{C}}$. Its restriction $\left.B\right|_{\mathfrak{u}}$ to $\mathfrak{u}$ is a real negative-definite symmetric bilinear form.

Let $G_{\mathbf{C}}$ be the connected group of complex matrices associated with the Lie algebra $\mathfrak{g}_{\mathbf{C}}$. Let $U, U_{\mathrm{ss}} \subset G_{\mathbf{C}}$ be the connected subgroup of $G_{\mathbf{C}}$ associated with the Lie algebras $\mathfrak{u},[\mathfrak{u}, \mathfrak{u}]$. If $G$ has a compact centre, by [K86, Propositions 5.3, 5.6], $G_{\mathbf{C}}$ is a reductive group with maximal compact subgroup $U$. By [K86, Theorem 4.32], $U_{\mathrm{ss}} \subset U$ is a semisimple compact subgroup, so that

$$
U=Z_{G}^{0} U_{\mathrm{ss}}
$$

By Weyl's theorem [K86, Theorem 4.26], the universal cover $\widetilde{U}_{\mathrm{ss}}$ of $U_{\mathrm{ss}}$ is compact. Set

$$
\widetilde{U}=Z_{G}^{0} \times \widetilde{U}_{s s}
$$

By (2.7) and (2.8), the obvious projection $\widetilde{U} \rightarrow U$ is a finite cover of $U$. 
Remark 2.2. By Weyl's unitary trick [K86, Proposition 5.7], if $G$ is semisimple and if $U$ is simply connected, it is equivalent to consider the finite dimensional complex representations of the Lie groups $G, U$ or of the Lie algebras $\mathfrak{g}, \mathfrak{u}$.

For general $G$, any finite dimensional complex representation of $G$ induces a representation of $\mathfrak{g}$. It extends uniquely to a representation of $\mathfrak{u}$. In generally, the $\mathfrak{u}$-representation does not alway lift to $U$. However, if $G$ has a compact centre, by (2.8), the $\mathfrak{u}$-representation lifts to $\widetilde{U}$.

Denote by $\operatorname{rk}_{\mathbf{C}}(G)$ (resp. $\left.\operatorname{rk}_{\mathbf{C}}(K)\right)$ the complex $\operatorname{rank}$ of $G$ (resp. $K$ ), i.e., the dimension of a Cartan subalgebra of $\mathfrak{g}_{\mathbf{C}}\left(\right.$ resp. $\left.\mathfrak{k}_{\mathbf{C}}\right)$.

Definition 2.3. The fundamental $\operatorname{rank}$ of $G$ is defined by

$$
\delta(G)=\operatorname{rk}_{\mathbf{C}}(G)-\operatorname{rk}_{\mathbf{C}}(K) \in \mathbf{N} .
$$

Note that $m$ and $\delta(G)$ have the same parity.

In the sequel, if $\gamma \in G$, we denote by $Z(\gamma) \subset G$ the centraliser of $\gamma$ in $G$, and by $\mathfrak{z}(\gamma) \subset \mathfrak{g}$ its Lie algebra. If $a \in \mathfrak{g}$, let $Z(a) \subset G$ be the stabiliser of $a$ in $G$, and let $\mathfrak{z}(a) \subset \mathfrak{g}$ be its Lie algebra. If $\mathfrak{a} \subset \mathfrak{g}$ is a subset, we define $Z(\mathfrak{a})$ and $\mathfrak{z}(\mathfrak{a})$ similarly.

2.2. The Casimir operator. Let $\mathscr{U}(\mathfrak{g})$ be the enveloping algebra of $\mathfrak{g}$, and let $\mathscr{Z}(\mathfrak{g}) \subset \mathscr{U}(\mathfrak{g})$ be the centre of $\mathscr{U}(\mathfrak{g})$.

Let $C^{\mathfrak{g}} \in \mathscr{Z}(\mathfrak{g})$ be the Casimir element associated to $B$. If $e_{1}, \cdots, e_{m}$ is an orthonormal basis of $\left(\mathfrak{p},\left.B\right|_{\mathfrak{p}}\right)$, and if $e_{m+1}, \cdots, e_{m+n}$ is an orthonormal basis of $\left(\mathfrak{k},-\left.B\right|_{\mathfrak{k}}\right)$. Then,

$$
C^{\mathfrak{g}}=-\sum_{i=1}^{m} e_{i}^{2}+\sum_{i=m+1}^{n+m} e_{i}^{2}
$$

If $V$ is a finite dimensional complex vector space, and if $\rho: \mathfrak{g} \rightarrow \operatorname{End}(V)$ is a morphism of Lie algebras, the map $\rho$ extends to a morphism $\mathscr{U}(\mathfrak{g}) \rightarrow \operatorname{End}(V)$ of algebras. We denote by $C^{\mathfrak{g}, V}$ or $C^{\mathfrak{g}, \rho} \in \operatorname{End}(V)$ the corresponding Casimir operator acting on $V$, i.e.,

$$
C^{\mathfrak{g}, V}=C^{\mathfrak{g}, \rho}=\rho\left(C^{\mathfrak{g}}\right) .
$$

Similarly, the Casimir of $\mathfrak{u}$ (with respect to $B$ ) acts on $V$, so that

$$
C^{\mathfrak{u}, V}=C^{\mathfrak{g}, V} .
$$

2.3. The symmetric space. We use the notation in Section 2.1. Let $\omega^{\mathfrak{g}}$ be the canonical left-invariant 1 -form on $G$ with values in $\mathfrak{g}$. By (2.1), $\omega^{\mathfrak{g}}$ splits as

$$
\omega^{\mathfrak{g}}=\omega^{\mathfrak{p}}+\omega^{\mathfrak{k}} .
$$

Let $X=G / K$ be the associated symmetric space. Let $p: G \rightarrow X$ be the natural projection. Then $p: G \rightarrow X$ is a $K$-principal bundle with connection form $\omega^{\mathfrak{k}}$. The group $K$ acts isometrically on $\mathfrak{p}$. The tangent bundle is given by

$$
T X=G \times_{K} \mathfrak{p} .
$$

By (2.14), the scalar product $\left.B\right|_{\mathfrak{p}}$ on $\mathfrak{p}$ induces a Riemannian metric $g^{T X}$ on $X$. The connection $\nabla^{T X}$ on $T X$ which is induced by $\omega^{\mathfrak{k}}$ is the Levi-Civita connection of $T X$. Its curvature is parallel and nonpositive. 
Let $e\left(T X, \nabla^{T X}\right) \in \Omega^{m}(X, o(T X))$ be the Euler characteristic form on $X$. Let $d v_{X} \in \Omega^{m}(X, o(T X))$ be the Riemannian volume form on the Riemannian manifold $\left(X, g^{T X}\right)$. Define $\left[e\left(T X, \nabla^{T X}\right)\right]^{\max } \in \mathbf{R}$ by

$$
e\left(T X, \nabla^{T X}\right)=\left[e\left(T X, \nabla^{T X}\right)\right]^{\max } d v_{X}
$$

An explicit formula for $\left[e\left(T X, \nabla^{T X}\right)\right]^{\max }$ can be found in [Sh18, (4-5)].

More generally, if $\tau$ is an orthogonal (resp. unitary) representation of $K$ on a finite dimensional Euclidean (resp. Hermitian) space $E_{\tau}$, set

$$
\mathscr{E}_{\tau}=G \times{ }_{K} E_{\tau}
$$

Then $\mathscr{E}_{\tau}$ is a Euclidean (resp. Hermitian) vector bundle on $X$, which is equipped with a connection induced by $\omega^{\mathfrak{k}}$.

We identify the space $C^{\infty}\left(X, \mathscr{E}_{\tau}\right)$ of smooth sections of $\mathscr{E}_{\tau}$ to the space $C^{\infty}\left(G, E_{\tau}\right)^{K}$ of smooth $E_{\tau}$-valued $K$-invariant functions on $G$. The group $G$ acts on the left on $C^{\infty}\left(X, \mathscr{E}_{\tau}\right)$. Denote by $C^{\mathfrak{g}, X, \tau}$ the Casimir element of $G$ on $C^{\infty}\left(X, \mathscr{E}_{\tau}\right)$. By (2.10), $C^{\mathfrak{g}, X, \tau}$ is a self-adjoint generalised Laplacian on $X$ satisfying (1.42). When $E_{\tau}=\Lambda^{\cdot}\left(\mathfrak{p}^{*}\right)$, we use the notation $C^{\mathfrak{g}, X}$. It is classical (see [B11, Proposition 7.8.1]) that $C^{\mathfrak{g}, X}$ is just the Hodge Laplacian on $X$ associated to the trivial line bundle.

2.4. Semisimple elements. The group $G$ acts isometrically on $X$. If $\gamma \in G$, let $d_{\gamma}$ be the corresponding displacement function on $X$ defined in (1.30). Also, $\ell_{\gamma}$ depends only on the conjugacy class of $\gamma$ in $G$, and will be denoted by $\ell_{[\gamma]}$. Let $X(\gamma) \subset X$ be the closed subset where $d_{\gamma}$ reaches its minimum. Clearly, the group $Z(\gamma)$ acts on $X(\gamma)$.

An element $\gamma \in G$ is called semisimple [E96, Definition 2.19.21], if $X(\gamma)$ is nonempty. If $\gamma$ is semisimple, by [B11, Theorem 3.1.2], there is $g_{\gamma} \in G$ such that $\gamma=g_{\gamma} e^{a} k^{-1} g_{\gamma}^{-1}$ and

$$
a \in \mathfrak{p}, \quad k \in K, \quad \operatorname{Ad}(k) a=a .
$$

Moreover, the norm $|a|$ depends only on the conjugacy class of $\gamma$ in $G$, and

$$
|a|=\ell_{[\gamma]}
$$

A semisimple element $\gamma$ is called elliptic, if $\ell_{[\gamma]}=0$.

If $\gamma$ is semisimple, by [K02, Proposition 7.25], $Z(\gamma)$ is a reductive group (with Cartan involution $g_{\gamma} \theta g_{\gamma}^{-1}$ ) with maximal compact subgroup $K(\gamma)$ with Cartan decomposition

$$
\mathfrak{z}(\gamma)=\mathfrak{p}(\gamma) \oplus \mathfrak{k}(\gamma)
$$

By [B11, Theorem 3.1.1], the map $g \in Z(\gamma) \rightarrow p g \in X$ induces the identification of $Z(\gamma)$-manifolds,

$$
Z(\gamma) / K(\gamma) \simeq X(\gamma)
$$


2.5. Semisimple orbital integrals. For $t>0$, let $\exp \left(-t C^{\mathfrak{g}, X, \tau}\right)$ be the heat operator of $C^{\mathfrak{g}, X, \tau}$. Let $p_{t}^{X, \tau}\left(x, x^{\prime}\right)$ be the smooth integral kernel of $\exp \left(-t C^{\mathfrak{g}, X, \tau}\right)$ with respect to the Riemannian volume $d v_{X}$.

Let $\gamma \in G$ be a semisimple element. Since $C^{\mathfrak{g}, X, \tau}$ commutes with the $G$-action on $C^{\infty}\left(X, \mathscr{E}_{\tau}\right)$, the function

$$
g \in G \rightarrow \operatorname{Tr}^{E_{\tau}}\left[p_{t}^{X, \tau}(p g, p \gamma g)\right] \in \mathbf{C}
$$

descends to $Z(\gamma) \backslash G$.

The form $-B(\cdot, \theta \cdot)$ induces a volume form $d v_{G}$ on $G$. We define $d v_{Z(\gamma)}$ similarly. Let $d v_{Z(\gamma) \backslash G}$ be the induced volume form on $Z(\gamma) \backslash G$, so that $d v_{G}=d v_{Z(\gamma) \backslash G} d v_{Z(\gamma)}$. In the same way, we can also define $d v_{K(\gamma) \backslash K}$ and its volume $\operatorname{vol}(K(\gamma) \backslash K)$.

Definition 2.4. Let $\gamma \in G$ be semisimple. The orbital integral of $\exp \left(-t C^{\mathfrak{g}, X, \tau}\right)$ is defined by

$$
\operatorname{Tr}^{[\gamma]}\left[\exp \left(-t C^{\mathfrak{g}, X, \tau}\right)\right]=\frac{1}{\operatorname{vol}(K(\gamma) \backslash K)} \int_{g \in Z(\gamma) \backslash G} \operatorname{Tr}^{E_{\tau}}\left[p_{t}^{X, \tau}(p g, p \gamma g)\right] d v_{Z(\gamma) \backslash G .}
$$

Clearly, $\operatorname{Tr}^{[\gamma]}\left[\exp \left(-t C^{\mathfrak{g}, X, \tau}\right)\right]$ depends only on the conjugacy class of $\gamma$ in $G$.

Remark 2.5. If $E_{\tau}$ is a $\mathbf{Z}_{2}$-graded or virtual representation of $K$, we use the notation $\operatorname{Tr}_{\mathrm{S}}^{[\gamma]}[\cdot]$ when the trace on the right-hand side of $(2.22)$ is replaced by the supertrace on $E_{\tau}$.

Remark 2.6. An explicit geometric formula for $\operatorname{Tr}^{[\gamma]}\left[\exp \left(-t C^{\mathfrak{g}, X, \tau}\right)\right]$ is obtained by Bismut [B11, Theorem 6.1.1]. This formula involves an explicit function $J_{\gamma}$ defined on the Lie algebra $\mathfrak{k}(\gamma)$. It can be written down with the help of a root system [BSh19a], [BSh19b, Theorem 4.7].

Remark 2.7. Most of the results obtained in [Sh18, ShY17] and as well as in this paper rely on Bismut's formula [B11, Theorem 6.1.1]. In this paper, the explicit formula for $J_{\gamma}$ is not needed, since all the involved orbital integrals have already been calculated in [Sh18, ShY17] except for a trivial one (5.23).

2.6. A discrete subgroup of $G$. Let $\Gamma \subset G$ be a discrete cocompact subgroup of $G$. By [Se60, Lemma 1] (see also [Ma19, Proposition 3.9]), $\Gamma$ contains only semisimple elements. Let $\Gamma_{e} \subset \Gamma$ be the subset of elliptic elements. Then, $\Gamma_{+}=$ $\Gamma-\Gamma_{e}$ consists of nonelliptic elements.

The group $\Gamma$ acts isometrically on the left on $X$. Take

$$
Z=\Gamma \backslash X=\Gamma \backslash G / K .
$$

Then $Z$ is a compact orbifold. We denote by $\widehat{p}: \Gamma \backslash G \rightarrow Z$ and $\widehat{\pi}: X \rightarrow Z$ the natural projections, so that the diagram

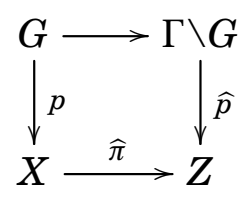

commutes. 
From now on until Section 6 , we assume that $\Gamma$ is torsion free, i.e., $\Gamma_{e}=\{\mathrm{id}\}$. Then $Z$ is a connected closed orientable Riemannian locally symmetric manifold with nonpositive sectional curvature. Since $X$ is contractible, $\pi_{1}(Z)=\Gamma$ and $X$ is the universal cover of $Z$. In Section 7, we will treat the case where $\Gamma$ is no longer torsion free.

The $\Gamma$-action on $X$ lifts to all the homogeneous Euclidean or Hermitian vector bundles $\mathscr{E}_{\tau}$ on $X$ constructed in (2.16), and preserves the metric connections. Then $\mathscr{E}_{\tau}$ descends to a Euclidean or Hermitian vector bundle

$$
\mathscr{F}_{\tau}=\Gamma \backslash \mathscr{E}_{\tau}=\Gamma \backslash G \times{ }_{K} E_{\tau}
$$

on $Z$, which is equipped with a canonical metric connection.

Let $F$ be a flat vector bundle on $Z$ with holonomy representation $\rho: \Gamma \rightarrow \operatorname{GL}_{r}(\mathbf{C})$, so that (1.33) holds. As in (1.43), we have the identification

$$
C^{\infty}\left(Z, \mathscr{F}_{\tau} \otimes F\right)=\left(C^{\infty}\left(X, \mathscr{E}_{\tau}\right) \otimes \mathbf{C}^{r}\right)^{\Gamma} .
$$

We use the notation in Section 1.5. In particular, the self-adjoint generalised Laplacian $C^{\mathfrak{g}, X, \tau} \otimes$ id descends to a generalised Laplacian operator $C^{\mathfrak{g}, Z, \tau, \rho}$ acting on $C^{\infty}\left(Z, \mathscr{F}_{\tau} \otimes F\right)$. As before, if $E_{\tau}=\Lambda^{*}\left(\mathfrak{p}^{*}\right)$, we denote $C^{\mathfrak{g}, Z, \rho}$ for simplification. Clearly, $C^{\mathfrak{g}, Z, \rho}$ is just the flat Laplacian introduced in Section 1.6.

For $\gamma \in \Gamma$, set

$$
\Gamma(\gamma)=Z(\gamma) \cap \Gamma
$$

By [Se60, Lemma2] (see also [Sh18, Proposition 4.9], [Ma19, Proposition 3.9]), $\Gamma(\gamma)$ is cocompact in $Z(\gamma)$.

Let $\left[\Gamma_{+}\right]$and $[\Gamma]$ be the sets of conjugacy classes in $\Gamma_{+}$and $\Gamma$. If $\gamma \in \Gamma$, the associated conjugacy class in $\Gamma$ is denoted by $[\gamma] \in[\Gamma] .^{3}$ If $[\gamma] \in[\Gamma]$, for all $\gamma^{\prime} \in[\gamma]$, the locally symmetric spaces

$$
\Gamma\left(\gamma^{\prime}\right) \backslash X\left(\gamma^{\prime}\right)
$$

are canonically diffeomorphic, which will be denoted by $B_{[\gamma]}$. Let $\operatorname{vol}\left(B_{[\gamma]}\right)$ be the Riemannian volume of $B_{[\gamma]}$ induced by the bilinear form $B$.

We have a generalisation of [Sh18, Theorem 4.10].

Theorem 2.8. There exist $c>0, C>0$ such that for $t>0$, we have

$$
\sum_{[\gamma] \in\left[\Gamma_{+}\right]} \operatorname{vol}\left(B_{[\gamma]}\right)|\operatorname{Tr}[\rho(\gamma)]|\left|\operatorname{Tr}^{[\gamma]}\left[\exp \left(-t C^{\mathfrak{g}, X, \tau}\right)\right]\right| \leqslant C \exp \left(-\frac{c}{t}+C t\right) .
$$

For $t>0$, the following identity holds,

$$
\operatorname{Tr}\left[\exp \left(-t C^{\mathfrak{g}, Z, \tau, \rho}\right)\right]=\sum_{[\gamma] \in[\Gamma]} \operatorname{vol}\left(B_{[\gamma]}\right) \operatorname{Tr}[\rho(\gamma)] \operatorname{Tr}^{[\gamma]}\left[\exp \left(-t C^{\mathfrak{g}, X, \tau}\right)\right] .
$$

Proof. Proceeding as the proof of the Selberg trace formula [Se56], by [B11, (4.8.8), (4.8.11), (4.8.16)], for $[\gamma] \in[\Gamma]$, we have

$$
\sum_{\gamma^{\prime} \in[\gamma]} \int_{x \in F_{Z}} \operatorname{Tr}^{E_{\tau}}\left[\gamma_{*}^{\prime} p_{t}^{X, \tau}\left(\left(\gamma^{\prime}\right)^{-1} x, x\right)\right] d v_{X}=\operatorname{vol}\left(B_{[\gamma]}\right) \operatorname{Tr}^{[\gamma]}\left[\exp \left(-t C^{\mathfrak{g}, X, \tau}\right)\right] .
$$

By Corollary 1.9, Proposition 1.10, and (2.31), we get our proposition.

\footnotetext{
${ }^{3}$ The quantities $\ell_{[\gamma]}$ and $\operatorname{Tr}^{[\gamma]}[\cdot]$ depend only on the conjugacy class of $\gamma$ in $G$. So they are well defined on the conjugacy classes of $\Gamma$.
} 
Remark 2.9. In [Mü11, Theorem 1.1], instead of heat operators, Müller obtain a similar formula for $\varphi\left(C^{\mathfrak{g}, Z, \tau, \rho}\right)$ where $\varphi$ is an even Paley-Wiener function on $\mathbf{R}$.

Let us give a direct application of the Selberg trace formula. Recall the following theorem due to [MoSt91, p. 194] and [B11, Theorem 7.9.1]. Let $N^{\Lambda^{*}\left(T^{*} X\right)}$ be the number operator on $\Lambda^{\cdot}\left(T^{*} X\right)$, which is multiplication by $p$ on $\Lambda^{p}\left(T^{*} X\right)$.

Theorem 2.10. If $\delta(G) \geqslant 2$, for any semisimple element $\gamma \in G$,

$$
\operatorname{Tr}_{\mathrm{s}}^{[\gamma]}\left[N^{\Lambda^{\bullet}\left(T^{*} X\right)} \exp \left(-t C^{\mathfrak{g}, X}\right)\right]=0 .
$$

We have a generalisation of [MoSt91, Corollary 2.2] and [B11, Theorem 7.9.3].

Corollary 2.11. Let $F$ be a flat vector bundle on $Z$ with holonomy $\rho$. Assume that $\operatorname{dim} Z$ is odd and that $\delta(G) \neq 1$. Then, for $t>0$, we have

$$
\operatorname{Tr}_{\mathrm{S}}\left[N^{\Lambda^{\bullet}\left(T^{*} Z\right)} \exp \left(-t \square^{Z}\right)\right]=0 .
$$

In particular,

$$
\prod_{i=1}^{m} \operatorname{det}^{*}\left(\left.\square^{Z}\right|_{\Omega^{i}(Z, F)}\right)^{(-1)^{i} i}=1 .
$$

For any acyclic and unitary representation $\rho_{0}$ of $\Gamma$, there is $\epsilon>0$ such that if $\rho$ is $C^{0} \epsilon$-close to $\rho_{0}$, then

$$
T_{\mathrm{CM}}(F)=1 .
$$

Proof. Since $m$ is odd, $\delta(G)$ is odd. Since $\delta(G) \neq 1$, we have $\delta(G) \geqslant 3$. By Theorems 2.8 and 2.10, we get (2.33) and (2.34). By Proposition 1.12, we get (2.35).

\section{Fundamental CARTAN Subalgebra AND RElated CONSTRUCtions}

The purpose of this section is to give a simple new conceptual proof for a complexified version of [Sh18, Theorem 6.11]. There, the corresponding proof is based on the classification theory of real simple Lie algebras.

This section is organised as follows. In Section 3.1, we introduce a $\theta$-invariant fundamental Cartan subalgebra $\mathfrak{h}=\mathfrak{b} \oplus \mathfrak{t}$.

In Section 3.2, we introduce a splitting of $\mathfrak{g}$ according to the action of $\mathfrak{b}$.

In Section 3.3, we introduce a root system of $\mathfrak{g}$ with respect to the fundamental Cartan subalgebra $\mathfrak{h}$. We reinterpret some objets constructed in Section 3.2.

Finally, in Section 3.4, we give a simple new conceptual proof for a complexified version of [Sh18, Theorem 6.11].

3.1. A fundamental Cartan subalgebra. Let $T \subset K$ be a maximal torus of $K$. Let $\mathfrak{t} \subset \mathfrak{k}$ be the Lie algebra of $T$. Set

$$
\mathfrak{b}=\{a \in \mathfrak{p}:[a, \mathfrak{t}]=0\} .
$$

Put

$$
\mathfrak{h}=\mathfrak{b} \oplus \mathfrak{t}
$$


By [K86, p. 129], $\mathfrak{h}$ is a Cartan subalgebra of $\mathfrak{g}$. Let $H \subset G$ be the associated Cartan subgroup, that is the centraliser of $\mathfrak{h}$ in $G$. By [K86, Theorem 5.22], $H$ is a connected abelian reductive subgroup of $G$, so that

$$
H=\exp (\mathfrak{b}) \times T \text {. }
$$

We will call $\mathfrak{h}$ and $H$ the fundamental Cartan subalgebra of $\mathfrak{g}$ and the fundamental Cartan subgroup of $G$.

Since $\mathfrak{h}_{\mathbf{C}}=\mathfrak{h} \otimes_{\mathbf{R}} \mathbf{C}$ is a Cartan subalgebra of $\mathfrak{g}_{\mathbf{C}}$, by (2.9) and (3.2), we have

$$
\delta(G)=\operatorname{dim} \mathfrak{b} .
$$

3.2. A splitting of $\mathfrak{g}$. Recall that $Z(\mathfrak{b}) \subset G$ is the stabiliser of $\mathfrak{b}$ in $G$ with Lie algebra $\mathfrak{z}(\mathfrak{b}) \subset \mathfrak{g}$. By [K02, Proposition 7.25], $Z(\mathfrak{b})$ is a possibly non connected reductive subgroup of $G$. Also, $\theta$ acts on $\mathfrak{z}(\mathfrak{b})$ so that we have the Cartan decomposition

$$
\mathfrak{z}(\mathfrak{b})=\mathfrak{p}(\mathfrak{b}) \oplus \mathfrak{k}(\mathfrak{b}) .
$$

Let $\mathfrak{m} \subset \mathfrak{z}(\mathfrak{b})$ be the orthogonal space (with respect to $B$ ) of $\mathfrak{b}$ in $\mathfrak{z}(\mathfrak{b})$. Then $\mathfrak{m}$ is a Lie subalgebra of $\mathfrak{g}$, and $\theta$ acts on $\mathfrak{m}$ so that

$$
\mathfrak{m}=\mathfrak{p}_{\mathfrak{m}} \oplus \mathfrak{k}_{\mathfrak{m}} .
$$

Let $M \subset G$ be the connected Lie group associated to the Lie algebra $\mathfrak{m}$. By [B11, (3.3.11) and Theorem 3.3.1], $M$ is closed in $G$ and is a connected reductive subgroup of $G$ with maximal compact subgroup

$$
K_{M}=M \cap K .
$$

Moreover, we have

$$
Z^{0}(\mathfrak{b})=\exp (\mathfrak{b}) \times M, \quad \mathfrak{z}(\mathfrak{b})=\mathfrak{b} \oplus \mathfrak{m}, \quad \mathfrak{p}(\mathfrak{b})=\mathfrak{b} \oplus \mathfrak{p}_{\mathfrak{m}}, \quad \mathfrak{k}(\mathfrak{b})=\mathfrak{k}_{\mathfrak{m}} .
$$

Since $\mathfrak{h} \subset \mathfrak{z}(\mathfrak{b})$ is also a fundamental Cartan subalgebra of $\mathfrak{z}(\mathfrak{b})$, we have

$$
\delta(M)=0 .
$$

Let

$$
X_{M}=M / K_{M}
$$

be the associated symmetric space. By (3.9), we see that $\operatorname{dim} X_{M}$ is even.

Let $\mathfrak{p}^{\perp}(\mathfrak{b}), \mathfrak{k}^{\perp}(\mathfrak{b}), \mathfrak{z}^{\perp}(\mathfrak{b})$ be respectively the orthogonal spaces (with respect to $B$ ) of $\mathfrak{p}(\mathfrak{b}), \mathfrak{k}(\mathfrak{b}), \mathfrak{z}(\mathfrak{b})$ in $\mathfrak{p}, \mathfrak{k}, \mathfrak{g}$. Clearly,

$$
\mathfrak{z}^{\perp}(\mathfrak{b})=\mathfrak{p}^{\perp}(\mathfrak{b}) \oplus \mathfrak{k}^{\perp}(\mathfrak{b}) .
$$

And also

$$
\mathfrak{p}=\mathfrak{b} \oplus \mathfrak{p}_{\mathfrak{m}} \oplus \mathfrak{p}^{\perp}(\mathfrak{b}), \quad \mathfrak{k}=\mathfrak{k}_{\mathfrak{m}} \oplus \mathfrak{k}^{\perp}(\mathfrak{b}), \quad \mathfrak{g}=\mathfrak{b} \oplus \mathfrak{m} \oplus \mathfrak{z}^{\perp}(\mathfrak{b}) .
$$

The group $K_{M}$ acts trivially on $\mathfrak{b}$. It also acts on $\mathfrak{p}_{\mathfrak{m}}, \mathfrak{p}^{\perp}(\mathfrak{b}), \mathfrak{k}_{\mathfrak{m}}$ and $\mathfrak{k}^{\perp}(\mathfrak{b})$, and preserves the splittings (3.12). Similarly, the groups $M$ and $Z^{0}(\mathfrak{b})$ act trivially on $\mathfrak{b}$, act on $\mathfrak{m}, \mathfrak{z}^{\perp}(\mathfrak{b})$, and preserves the third splitting in (3.12). 
3.3. A root system of $(\mathfrak{h}, \mathfrak{g})$. Let $R \subset \mathfrak{h}_{\mathbf{C}}^{*}$ be a root system of $(\mathfrak{h}, \mathfrak{g})$ [K02, Section II.4]. If $\alpha \in R$, let $\mathfrak{g}_{\alpha} \subset \mathfrak{g}_{\mathbf{C}}$ be the weight space associated with $\alpha$, which is of dimension 1 . Then we have the splitting

$$
\mathfrak{g}_{\mathbf{C}}=\mathfrak{h}_{\mathbf{C}} \bigoplus \oplus_{\alpha \in R} \mathfrak{g}_{\alpha} .
$$

If $\alpha \in R$, then $\bar{\alpha} \in R$. A root is called real if $\bar{\alpha}=\alpha$, imaginary if $\bar{\alpha}=-\alpha$, and complex otherwise. By [K02, Proposition 11.16] (see also [BSh19b, Proposition 3.7]), since $\mathfrak{h}$ is fundamental, there are no real roots. Let $R^{\mathrm{im}} \subset R$ and $R^{\mathrm{c}} \subset R$ be the subsets of imaginary and complex roots, so that

$$
R=R^{\mathrm{im}} \sqcup R^{\mathrm{c}} .
$$

Let $\mathfrak{h}^{\perp}$ be the orthogonal to $\mathfrak{h}$ in $\mathfrak{g}$ with respect to $B$. Set

$$
\mathfrak{i}=\mathfrak{z}(\mathfrak{b}) \cap \mathfrak{h}^{\perp} \text {. }
$$

Let $\mathfrak{c}$ be the orthogonal to $i$ in $\mathfrak{h}^{\perp}$. Then

$$
\mathfrak{g}=\mathfrak{h} \oplus \mathfrak{i} \oplus \mathfrak{c} .
$$

By (3.13), we have

$$
\mathfrak{i}_{\mathbf{C}}=\oplus_{\alpha \in R^{\text {im }}} \mathfrak{g}_{\alpha}, \quad \mathfrak{c}_{\mathbf{C}}=\oplus_{\alpha \in R^{\mathrm{c}}} \mathfrak{g}_{\alpha} .
$$

Also, $\theta$ acts on $\mathfrak{i}, \mathfrak{c}$, so that we have the splittings

$$
\mathfrak{i}=\mathfrak{i}_{\mathfrak{p}} \oplus \mathfrak{i}_{\mathfrak{k}}, \quad \mathfrak{c}=\mathfrak{c}_{\mathfrak{p}} \oplus \mathfrak{c}_{\mathfrak{k}} .
$$

By (3.5), (3.6), (3.12), (3.15), and (3.16), we have

$$
\begin{aligned}
\mathfrak{m}=\mathfrak{t} \oplus \mathfrak{i}, & \mathfrak{p}_{\mathfrak{m}}=\mathfrak{i}_{\mathfrak{p}}, & \mathfrak{k}_{\mathfrak{m}}=\mathfrak{t} \oplus \mathfrak{i}_{\mathfrak{k}}, \\
\mathfrak{z}^{\perp}(\mathfrak{b})=\mathfrak{c}, & \mathfrak{p}^{\perp}(\mathfrak{b})=\mathfrak{c}_{\mathfrak{p}}, & \mathfrak{k}^{\perp}(\mathfrak{b})=\mathfrak{c}_{\mathfrak{k} .}
\end{aligned}
$$

In particular, we can rewrite the third identity of (3.12),

$$
\mathfrak{g}=\mathfrak{b} \oplus \mathfrak{m} \oplus \mathfrak{c} .
$$

Proposition 3.1. The vector spaces $\mathfrak{i}_{\mathfrak{p}}, \mathfrak{i}_{\mathfrak{k}}$ have even dimensions, and $\mathfrak{c}_{\mathfrak{p}}, \mathfrak{c}_{\mathfrak{k}}$ have the

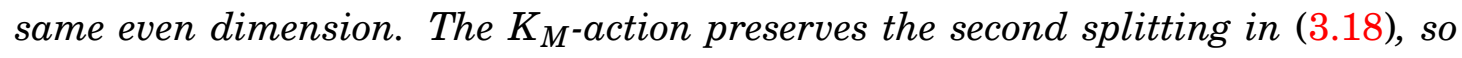
that the actions on $\mathfrak{c}_{\mathfrak{p}}, \mathfrak{c}_{\mathfrak{k}}$ are equivalent.

Proof. This is [BSh19b, Proposition 3.8], except in the last statement the group $K_{M}$ is replaced by $T$. By the consideration after (3.12), by the last two identities in (3.19), $K_{M}$ acts on $\mathfrak{c}_{\mathfrak{p}}, \mathfrak{c}_{\mathfrak{k}}$. Since $K_{M}$ and $\mathfrak{b}$ commutes, if $b \in \mathfrak{b}$ is such that $\langle\alpha, b\rangle \neq 0$ for all $\alpha \in R^{\mathrm{c}}$, then $\operatorname{ad}(b): \mathfrak{c}_{\mathfrak{p}} \rightarrow \mathfrak{c}_{\mathfrak{k}}$ defines a $K_{M}$-equivalent.

Let $R_{+} \subset R$ be a positive root system. Set

$$
R_{+}^{\mathrm{im}}=R_{+} \cap R^{\mathrm{im}}, \quad \quad R_{+}^{\mathrm{c}}=R_{+} \cap R^{\mathrm{c}} .
$$

As explained in [BSh19b, Section 3.5], we can choose $R_{+}$so that $R_{+}^{\mathrm{c}}$ is preserved by the complex conjugation.

Set

$$
\mathfrak{c}_{+}, \mathbf{C}=\oplus_{\alpha \in R_{+}^{c}} \mathfrak{g}_{\alpha}, \quad \mathfrak{c}_{-, \mathbf{C}}=\oplus_{\alpha \in R_{-}^{c}} \mathfrak{g}_{\alpha} .
$$

Proposition 3.2. The following statements hold. 
i) The vector spaces $\mathfrak{c}_{+}, \mathbf{C}, \mathfrak{c}_{-}, \mathbf{C}$ are the complexifications of real Lie subalgebras $\mathfrak{c}_{+}, \mathfrak{c}_{-}$of $\mathfrak{g}$, which have the same even dimension, and are such that

$$
\mathfrak{c}=\mathfrak{c}_{+} \oplus \mathfrak{c}_{-}, \quad \mathfrak{c}_{+}=\theta \mathfrak{c}_{-} .
$$

ii) The bilinear form $B$ vanishes on $\mathfrak{c}_{+}, \mathfrak{c}_{-}$and induces the identification,

$$
\mathfrak{c}_{-}^{*} \simeq \mathfrak{c}_{+} .
$$

iii) The group $Z^{0}(\mathfrak{b})$ acts on $\mathfrak{c}_{ \pm}$, so that (3.24) is $Z^{0}(\mathfrak{b})$-equivalent.

iv) The actions of $M$ on $\mathfrak{c}_{ \pm}$are equivalent.

v) The projections on $\mathfrak{p}, \mathfrak{k}$ map $\mathfrak{c}_{ \pm}$into $\mathfrak{c}_{\mathfrak{p}}, \mathfrak{c}_{\mathfrak{k}}$ isomorphically.

vi) Finally, the actions of $K_{M}$ on $\mathfrak{c}_{+}, \mathfrak{c}_{-}, \mathfrak{c}_{\mathfrak{p}}, \mathfrak{c}_{\mathfrak{k}}$ are equivalent.

Proof. The statements i), ii), v) are just [BSh19b, Proposition 3.10], and vi) has been established for the $T$-action instead of the $K_{M}$-action .

By our choice of the positive root system, we have

$$
\left[\mathfrak{i}, \mathfrak{c}_{ \pm}, \mathbf{C}\right] \subset \mathfrak{c}_{ \pm}, \mathbf{C} .
$$

Therefore, $\mathfrak{z}(\mathfrak{b})=\mathfrak{h} \oplus \mathfrak{i}$ preserves $\mathfrak{c}_{ \pm}, \mathbf{C}$, and $Z^{0}(\mathfrak{b})$ acts on $\mathfrak{c}_{ \pm, \mathbf{C}}$. Since the $Z^{0}(\mathfrak{b})$-action on $\mathfrak{c}_{ \pm}, \mathbf{C}$ commutes with the complex conjugation, $Z^{0}(\mathfrak{b})$ acts on $\mathfrak{c}_{ \pm}$. Since $B$ is $Z^{0}(\mathfrak{b})$-invariant, we see that (3.24) is $Z^{0}(\mathfrak{b})$-equivalent, from which we get iii).

Since $\delta(M)=0$, by [K86, Probleme XII.14], there is $k_{0} \in K_{M}$ such that $\left.\operatorname{Ad}\left(k_{0}\right)\right|_{\mathfrak{m}}=$ $\left.\theta\right|_{\mathfrak{m}}$. By the second identity of (3.23), $\operatorname{Ad}\left(k_{0}\right) \theta: \mathfrak{c}_{+} \rightarrow \mathfrak{c}_{-}$is an equivalence of $M$ representations, from which we get iv).

Since the projection $\mathfrak{c}_{ \pm} \rightarrow \mathfrak{c}_{\mathfrak{p}}$ is $(1+\theta) / 2$, since $K_{M}$ is fixed by $\theta$, we get vi).

Corollary 3.3. For $0 \leqslant j \leqslant \operatorname{dim} \mathfrak{c}_{ \pm}$, we have isomorphisms of representations of $M$,

$$
\Lambda^{j}\left(\mathfrak{c}_{ \pm}^{*}\right) \simeq \Lambda^{\operatorname{dim} \mathfrak{c}_{ \pm}-j}\left(\mathfrak{c}_{ \pm}^{*}\right) .
$$

Proof. Since $\delta(M)=0, M$ has a compact center. By Proposition 2.1, $M$ acts trivially on $\Lambda^{\operatorname{dim} \mathfrak{c}_{ \pm}}\left(\mathfrak{c}_{ \pm}^{*}\right)$. We have an isomorphism of real representations of $M$,

$$
\Lambda^{j}\left(\mathfrak{c}_{ \pm}^{*}\right) \simeq \Lambda^{\operatorname{dim} \mathfrak{c}_{ \pm}-j}\left(\mathfrak{c}_{ \pm}\right) .
$$

By Proposition 3.2 iii) iv) and (3.27), we get (3.26).

3.4. A lifting property. Let $R(K)$ be the representation ring of $K$. We can identify $R(K)$ with the subring of the $\operatorname{Ad}(K)$-invariant smooth functions on $K$ which is generated by the characters of finite dimensional complex representations of $K$.

The restriction induces an injective morphism of rings

$$
i: R(K) \rightarrow R(T) .
$$

Let $W(T: K)=N_{K}(T) / T$ be the Weyl group of $K$, where $N_{K}(T)$ is the normaliser of $T$ in $K$. Then $W(T: K)$ acts on $R(T)$. By [BrDi85, Proposition VI.2.1], $i$ induces an isomorphism of rings

$$
i: R(K) \simeq R(T)^{W(T: K)} .
$$

Proposition 3.4. The adjoint action of $N_{K}(T)$ preserves the decomposition

$$
\mathfrak{g}=\mathfrak{t} \oplus \mathfrak{b} \oplus \mathfrak{i}_{\mathfrak{k}} \oplus \mathfrak{i}_{\mathfrak{p}} \oplus \mathfrak{c}_{\mathfrak{k}} \oplus \mathfrak{c}_{\mathfrak{p}}
$$


Proof. Since $N_{K}(T)$ preserves $\mathfrak{t}$, by (3.1), $N_{K}(T)$ preserves $\mathfrak{b}$. Also, $N_{K}(T)$ preserves $\mathfrak{z}(\mathfrak{b})$. Since $N_{K}(T) \subset K$ preserves $B$, by (3.15), $N_{K}(T)$ preserves i. Using again $N_{K}(T)$ preserves $B$, by (3.16), $N_{K}(T)$ preserves c. By (3.18), $N_{K}(T)$ preserves $\mathfrak{i}_{\mathfrak{p}}, \mathfrak{i}_{\mathfrak{k}}, \mathfrak{c}_{\mathfrak{p}}, \mathfrak{c}_{\mathfrak{k}}$.

Theorem 3.5. The adjoint action of $T$ on $\mathfrak{i}_{\mathfrak{p}, \mathbf{C}}, \mathfrak{i}_{\mathfrak{k}, \mathbf{C}}, \mathfrak{c}_{\mathfrak{p}, \mathbf{C}} \simeq \mathfrak{c}_{\mathfrak{k}, \mathbf{C}} \simeq \mathfrak{c}_{+, \mathbf{C}} \simeq \mathfrak{c}_{-, \mathbf{C}}$ lift uniquely to virtual representations in $R(K)$.

Proof. By Proposition 3.4, the characters of $T$ on $\mathfrak{i}_{\mathfrak{p}, \mathbf{C}}, \mathfrak{i}_{\mathfrak{k}, \mathbf{C}}$, and $\mathfrak{c}_{\mathfrak{p}, \mathbf{C}}$ are $W(T: K)$ invariant. By (3.28) and (3.29), the $T$-actions on $\mathfrak{i}_{\mathfrak{p}, \mathbf{C}}, \mathfrak{i}_{\mathfrak{k}, \mathbf{C}}$, and $\mathfrak{c}_{\mathfrak{p}, \mathbf{C}}$ lift uniquely to $R(K)$.

Corollary 3.6. For $i, j \in \mathbf{N}$, the adjoint representations of $K_{M}$ on $\Lambda^{i}\left(\mathfrak{p}_{\mathfrak{m}, \mathbf{C}}^{*}\right)$ and $\Lambda^{j}\left(\mathfrak{c}_{ \pm, \mathbf{C}}^{*}\right)$ have unique lifts in $R(K)$.

Proof. This is a consequence of (3.19) and Theorem 3.5,

Remark 3.7. Let $R O(K)$ be the real representation ring of $K$. By [BrDi85, Proposition II.7.8], the complexification $V \in R O(K) \rightarrow V \otimes_{\mathbf{R}} \mathbf{C} \in R(K)$ induces an injective morphism of rings,

$$
R O(K) \rightarrow R(K)
$$

In [Sh18, Theorem 6.11], using the classification theory on the real simple Lie algebras, we have shown that when $\delta(G)=1$, the real representation $\mathfrak{c}_{ \pm}$of $K_{M}$ has a unique lift in $R O(K)$. However, the above complexified version is enough for applications both in [Sh18] and the current paper.

\section{THE RUELlE DYNAMICAL ZETA FUNCTIONS FOR ARBITRARY TWIST}

The purpose of this section is to introduce the Ruelle dynamical zeta function for arbitrary twist. We restate the main result of this article as Theorem 4.3. Its proof will be given in Sections 5 and 6. Theorem 4.3 generalises author's previous result [Sh18, Theorem 1.1] where $\rho$ is unitary, as well as the results on hyperbolic manifolds due to Müller [Mü20, Theorem 1.1, Proposition 1.3] and Spilioti [Sp18, Theorem 1.2], [Sp20b, Theorem 2].

We use the notation in Section 2 . Recall that $Z=\Gamma \backslash G / K$ is a locally symmetric manifold and $\rho: \Gamma \rightarrow \mathrm{GL}_{r}(\mathbf{C})$ is a representation of $\Gamma$.

Let us recall the definition of the Ruelle dynamical zeta function introduced by Fried [F87, Section 5]. For $[\gamma] \in[\Gamma]$, recall that $B_{[\gamma]}$ (see (2.28)) is the locally symmetric space $\Gamma(\gamma) \backslash X(\gamma)$. By [DuKVa79, Proposition 5.15], the set of nontrivial closed geodesics on $Z$ consists of a disjoint union

$$
\coprod_{[\gamma] \in\left[\Gamma_{+}\right]} B_{[\gamma]} .
$$

Moreover, if $[\gamma] \in\left[\Gamma_{+}\right]$, all the elements of $B_{[\gamma]}$ have the same length $\ell_{[\gamma]}>0$.

If $[\gamma] \in\left[\Gamma_{+}\right]$, the geodesic flow induces a locally free action of $\mathbb{S}^{1}$ on $B_{[\gamma]}$, so that $B_{[\gamma]} / \mathbb{S}^{1}$ is a closed orbifold. Let $\chi_{\text {orb }}\left(B_{[\gamma]} / \mathbb{S}^{1}\right) \in \mathbf{Q}$ be the orbifold Euler characteristic number [Sa57]. We refer the reader to [Sh18, Proposition 5.1] for an explicit 
formula for $\chi_{\mathrm{orb}}\left(B_{[\gamma]} / \mathbb{S}^{1}\right)$. In particular, if $\delta(G) \geqslant 2$, or if $\delta(G)=1$ and $\gamma$ can not be conjugate by an element of $G$ into the fundamental Cartan subgroup $H$, then

$$
\chi_{\text {orb }}\left(B_{[\gamma]} / \mathbb{S}^{1}\right)=0 .
$$

The $\mathbb{S}^{1}$-action on $B_{[\gamma]}$ is not necessarily effective. Let

$$
m_{[\gamma]}=\left|\operatorname{ker}\left(\mathbb{S}^{1} \rightarrow \operatorname{Diff}\left(B_{[\gamma]}\right)\right)\right| \in \mathbf{N}^{*}
$$

be the generic multiplicity.

By [Sh18, Theorem 5.6] and by (1.34), there is $\sigma_{0}>0$ such that

$$
\sum_{[\gamma] \in\left[\Gamma_{+}\right]} \frac{\left|\chi_{\mathrm{orb}}\left(B_{[\gamma]} / \mathbb{S}^{1}\right)\right|}{m_{[\gamma]}}|\operatorname{Tr}[\rho(\gamma)]| e^{-\sigma_{0} \ell_{[\gamma]}}<\infty .
$$

Definition 4.1. For $\operatorname{Re}(\sigma) \geqslant \sigma_{0}$, set

$$
R_{\rho}(\sigma)=\exp \left(\sum_{[\gamma] \in\left[\Gamma_{+}\right]} \frac{\chi_{\mathrm{orb}}\left(B_{[\gamma]} / \mathbb{S}^{1}\right)}{m_{[\gamma]}} \operatorname{Tr}[\rho(\gamma)] e^{-\sigma \ell_{[\gamma]}}\right) .
$$

Remark 4.2. By (4.2), if $\delta(G) \geqslant 2$, the dynamical zeta function $R_{\rho}(\sigma)$ is the constant function 1. Moreover, if $\delta(G)=1$, then the sum on the right-hand side of (4.5) can be reduced to a sum over $[\gamma] \in\left[\Gamma_{+}\right]$such that $\gamma$ can be conjugate into $H$.

Recall that $\square^{Z}$ is the flat Laplacian on $Z$. We restate Theorem 0.1 as follows.

Theorem 4.3. Assume that $\operatorname{dim} Z$ is odd. The following statements hold.

i) The dynamical zeta function $R_{\rho}(\sigma)$ has a meromorphic extension to $\sigma \in \mathbf{C}$.

ii) There exist explicit constants $C_{\rho} \in \mathbf{R}^{*}$ and $r_{\rho} \in \mathbf{Z}$ (see (5.33) and (5.35)) such that when $\sigma \rightarrow 0$, we have

$$
R_{\rho}(\sigma)=C_{\rho}\left\{\prod_{i=1}^{m}\left(\operatorname{det}^{*}\left(\left.\square^{Z}\right|_{\Omega^{i}(Z, F)}\right)\right)^{(-1)^{i} i}\right\} \sigma^{r_{\rho}}+\mathscr{O}\left(\sigma^{r_{\rho}+1}\right) .
$$

iii) There is $k \in \mathbf{N}$ such that for any acyclic and unitary representation $\rho_{0}$ of $\Gamma$, there exists $\epsilon>0$ such that if $\rho$ is $C^{k} \epsilon$-close to $\rho_{0}$, then $\square^{Z}$ is invertible and

$$
C_{\rho}=1, \quad r_{\rho}=0,
$$

so that

$$
R_{\rho}(0)=T_{\mathrm{CM}}(F)
$$

Proof. Since $m$ and $\delta(G)$ have the same parity, we have $\delta(G) \geqslant 1$ is odd. When $\delta(G) \geqslant 3$, our theorem with $k=0$ follows from Corollary 2.11 and Remark 4.2. If $\delta(G)=1$, the proofs of i), ii) as well as the proof of iii) when $Z_{G}$ is noncompact are based on the introducing of the Selberg zeta functions with arbitrary twist and will be given in Section 5.4. The proof of iii) when $Z_{G}$ is compact will be given in Section 6.3.

Let $\bar{R}_{\rho}$ be the meromorphic function defined for $\sigma \in \mathbf{C}$ by

$$
\bar{R}_{\rho}(\sigma)=\overline{R_{\rho}(\bar{\sigma})}
$$

Proposition 4.4. The following identities of meromorphic functions on $\mathbf{C}$ hold,

$$
R_{\rho^{*}}(\sigma)=R_{\rho}(\sigma), \quad R_{\bar{\rho}}(\sigma)=\bar{R}_{\rho}(\sigma) .
$$


Proof. The second identity in (4.10) is trivial. Let us show the first one. We have

$$
\operatorname{Tr}\left[\rho^{*}(\gamma)\right]=\operatorname{Tr}\left[\rho\left(\gamma^{-1}\right)\right] .
$$

Note that $\gamma \rightarrow \gamma^{-1}$ induces a bijection on $\left[\Gamma_{+}\right]$. Since $d_{\gamma^{-1}}(x)=d_{\gamma}(x)$, we have

$$
\ell_{\left[\gamma^{-1}\right]}=\ell_{[\gamma]}, \quad X\left(\gamma^{-1}\right)=X(\gamma),
$$

By (4.12), using $\Gamma\left(\gamma^{-1}\right)=\Gamma(\gamma)$, we get

$$
B_{\left[\gamma^{-1}\right]}=B_{[\gamma]}, \quad \quad m_{\left[\gamma^{-1}\right]}=m_{[\gamma]} .
$$

By (4.5) and (4.11)-(4.13), we get the first identity in (4.10).

\section{The SELBERG ZETA FUnCTION FOR ARBITRARY TWIST}

The purpose of this section is to extend the results of [Sh18, Theorems 7.6 and 7.7] on the zeta functions of Ruelle and Selberg to arbitrary twist with the help of the Müller's Selberg trace formula (Theorem 2.8), inexplicitly of Bismut's orbital integral formula [B11, Theorem 6.1.1], and of the lifting properties (Theorem 3.5, Corollary 3.6).

More precisely, in [Sh18, Section 7], if $\delta(G)=1$, we associate a Selberg zeta function $Z_{\eta, \rho}$ to a representation $\eta$ of $M$ satisfying [Sh18, Assumption 7.1] and to a unitary representation $\rho$ of $\Gamma$. Moreover, we show that $Z_{\eta, \rho}$ has a meromorphic extension to $\mathbf{C}$ and satisfies a functional equation. Also, we prove that the Ruelle zeta function with a unitary twist is an alternating product of certain Selberg zeta functions. In this way, we obtained the meromorphic extension of the Ruelle zeta function. In this section, we extend all the above results to arbitrary $\rho$ and to a slightly larger class of $\eta$.

This section is organised as follows. In Section 5.1, we recall some results on the structure of real reductive groups with $\delta(G)=1$ obtained in [Sh18, Section 6].

In Sections 5.2 and 5.3, when $\delta(G)=1$, we introduce a class of virtual representations $\eta$ of the group $M$. If $\rho: \Gamma \rightarrow \mathrm{GL}_{r}(\mathbf{C})$ is a representation of $\Gamma$, we introduce the Selberg zeta function $Z_{\eta, \rho}$ associated to $(\eta, \rho)$. We show the meromorphic extension and a functional equation for $Z_{\eta, \rho}$.

Finally, in Section 5.4, we show that $R_{\rho}$ is an alternating product of certain Selberg zeta functions. In particular, we show Theorem 4.3 i) ii), as well as iii) when $Z_{G}$ is non compact.

In the whole section, we assume $\delta(G)=1$.

5.1. The structure of the reductive group $G$ with $\delta(G)=1$. We use the notation in Sections 2 and 3. Assume that $\delta(G)=1$. To make this section readable, instead of writing $\mathfrak{c}_{ \pm}$, we use notation as [Sh18], i.e.,

$$
\mathfrak{n}=\mathfrak{c}_{+}, \quad \overline{\mathfrak{n}}=\mathfrak{c}_{-} .
$$

When $G$ has a non compact centre, thanks to the following proposition, $G$ has a very simple structure.

Proposition 5.1. If $G$ has a non compact centre, then

$$
G=\exp (\mathfrak{b}) \times M, \quad \mathfrak{z}^{\perp}(\mathfrak{b})=0 .
$$


Proof. Since $G$ has a non compact centre, we have $\operatorname{dim} \mathfrak{z}_{\mathfrak{p}} \geqslant 1$. Since $\mathfrak{z}_{\mathfrak{p}} \subset \mathfrak{b}$ and since $\operatorname{dim} \mathfrak{b}=1$, we have $\mathfrak{z}_{\mathfrak{p}}=\mathfrak{b}$. So $\mathfrak{g}=\mathfrak{z}(\mathfrak{b})$. By (3.8), we get (5.2).

Assume in the rest of this subsection that $G$ has a compact centre. By Propositions 3.1 and 3.2 i), dimn is even. Set

$$
\ell=\frac{1}{2} \operatorname{dim} \mathfrak{n}
$$

Since $G$ has a compact centre, we have $\mathfrak{b} \not \subset \mathfrak{z} \mathfrak{g}$. Therefore, $\mathfrak{z}^{\perp}(\mathfrak{b}) \neq 0$ and $\ell>0$. Recall that we have fixed a system of positive roots $R_{+}$.

Proposition 5.2. There is $\alpha_{0} \in \mathfrak{b}^{*}$ such that for all $\alpha \in R_{+}^{\mathrm{c}}$,

$$
\left.\alpha\right|_{\mathfrak{b}}=\alpha_{0}
$$

Equivalently, $\mathfrak{b}$ acts on $\mathfrak{n}$ and $\overline{\mathfrak{n}}$ by $\pm \alpha_{0} \in \mathfrak{b}^{*}$, i.e., for $a \in \mathfrak{b}, f \in \mathfrak{n}, \bar{f} \in \overline{\mathfrak{n}}$, we have

$$
[a, f]=\left\langle\alpha_{0}, a\right\rangle f, \quad[a, \bar{f}]=-\left\langle\alpha_{0}, a\right\rangle \bar{f} .
$$

In particular,

$$
[\mathfrak{n}, \overline{\mathfrak{n}}] \subset \mathfrak{z}(\mathfrak{b}), \quad[\mathfrak{n}, \mathfrak{n}]=[\overline{\mathfrak{n}}, \overline{\mathfrak{n}}]=0,
$$

and

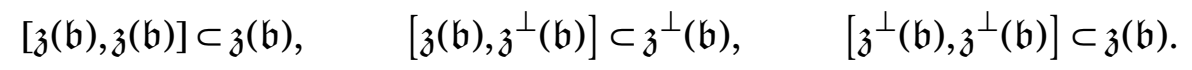

Proof. This is [Sh18, Propositions 6.2 and 6.3].

Let $\mathfrak{u}(\mathfrak{b}) \subset \mathfrak{u}, \mathfrak{u}_{\mathfrak{m}} \subset \mathfrak{u}$ be the compact forms of $\mathfrak{z}(\mathfrak{b})$ and $\mathfrak{m}$. Then

$$
\mathfrak{u}(\mathfrak{b})=\sqrt{-1} \mathfrak{b} \oplus \mathfrak{u}_{\mathfrak{m}}, \quad \quad \mathfrak{u}_{\mathfrak{m}}=\sqrt{-1} \mathfrak{p}_{\mathfrak{m}} \oplus \mathfrak{k}_{\mathfrak{m}}
$$

Let $\mathfrak{u}^{\perp}(\mathfrak{b})$ be the orthogonal space of $\mathfrak{u}(\mathfrak{b})$ in $\mathfrak{u}$ with respect to $-\left.B\right|_{\mathfrak{u}}$. Then,

$$
\mathfrak{u}^{\perp}(\mathfrak{b})=\sqrt{-1} \mathfrak{p}^{\perp}(\mathfrak{b}) \oplus \mathfrak{k}^{\perp}(\mathfrak{b}) .
$$

By (5.7), we have

$$
[\mathfrak{u}(\mathfrak{b}), \mathfrak{u}(\mathfrak{b})] \subset \mathfrak{u}(\mathfrak{b}), \quad\left[\mathfrak{u}(\mathfrak{b}), \mathfrak{u}^{\perp}(\mathfrak{b})\right] \subset \mathfrak{u}^{\perp}(\mathfrak{b}), \quad\left[\mathfrak{u}^{\perp}(\mathfrak{b}), \mathfrak{u}^{\perp}(\mathfrak{b})\right] \subset \mathfrak{u}(\mathfrak{b}) .
$$

Thus, $(\mathfrak{u}, \mathfrak{u}(\mathfrak{b}))$ is a compact symmetric pair.

Let $U(\mathfrak{b}) \subset U, U_{M} \subset U, A_{0} \subset U$ be the connected subgroups of $U$ associated to the Lie subalgebra $\mathfrak{u}(\mathfrak{b}), \mathfrak{u}_{\mathfrak{m}}, \sqrt{-1} \mathfrak{b}$ of $\mathfrak{u}$, so that

$$
U(\mathfrak{b})=A_{0} U_{M}
$$

Remark 5.3. All of the above three groups are compact. Indeed, $U(\mathfrak{b})$ is the stabiliser of $\sqrt{-1} \mathfrak{b}$ in $U$, which is compact in $U$. Since $\delta(M)=0, M$ has compact centre. Therefore, $U_{M}$ is the compact form of $M$. The compactness of $A_{0}$ is established in [Sh18, Proposition 6.6] 
5.2. The Selberg zeta function. Recall that $K_{M}$ and $K$ have the maximal torus $T$. By (3.29), the restriction to $K_{M}$ induces an injective morphism of rings $R(K) \rightarrow$ $R\left(K_{M}\right)$.

Assumption 5.4. Assume that $\eta=\eta^{+}-\eta^{-}$is a virtual $M$-representation on the finite dimensional complex vector space $E_{\eta}=E_{\eta}^{+}-E_{\eta}^{+}$such that

(1) $\left.\eta\right|_{K_{M}}=\left.\eta^{+}\right|_{K_{M}}-\left.\eta^{-}\right|_{K_{M}} \in R\left(K_{M}\right)$ has a unique lift in $R(K)$.

(2) the Casimir $C^{\mathfrak{u}_{\mathfrak{m}}}$ of $\mathfrak{u}_{\mathfrak{m}}$ acts on $\eta^{ \pm}$by the same scalar $C^{\mathfrak{u}_{\mathfrak{m}}, \eta} \in \mathbf{R}$.

Remark 5.5. If $Z_{G}$ is non compact, then $K_{M}=K$. Assumption (1) is empty.

Remark 5.6. If $Z_{G}$ is compact, Assumption 5.4 is slightly different from [Sh18, Assumption 7.1]. Indeed, Assumption 5.4 is the complexified virtual version [Sh18, Assumption 7.1 (1) (3)]. Moreover, the statement (2) in [Sh18, Assumption 7.1], which requires the $\mathfrak{u}_{\mathfrak{m}}$-action on $\eta$ lifts to $U_{M}$, is a technique condition. Since $\delta(M)=0, M$ has a compact centre. By Remark 2.2, the $\mathfrak{u}_{\mathfrak{m}}$-action on $\eta$ lifts to a finite cover of $U_{M}$. Thanks to this observation, most of arguments (see also Remark 5.9 and Section 5.3.2) go though up to evident modifications.

Recall that $\rho: \Gamma \rightarrow \mathrm{GL}_{r}(\mathbf{C})$ is a representation of $\Gamma$. Following [Sh18, Definition $7.4]$, let us define the Selberg zeta function associated to the pair $(\eta, \rho)$. Recall that $H=\exp (\mathfrak{b}) \times T$ is the fundamental Cartan subgroup of $G$. For $e^{a} k^{-1} \in H$, we write $\gamma \sim e^{a} k^{-1}$ if there is $g_{\gamma} \in G$ such that $\gamma=g_{\gamma} e^{a} k^{-1} g_{\gamma}^{-1}$. By [Sh18, (7-62)] and by (1.34), there is $\sigma_{1}>0$ such that

$$
\sum_{\substack{[\gamma] \in\left[\Gamma_{+}\right] \\ \gamma \sim e^{a} k^{-1} \in H}} \frac{\left|\chi_{\text {orb }}\left(B_{[\gamma]} / \mathbb{S}^{1}\right)\right|}{m_{[\gamma]}}|\operatorname{Tr}[\rho(\gamma)]| \frac{e^{-\sigma_{1} \ell_{[\gamma]}}}{\left.\left|\operatorname{det}\left(1-\operatorname{Ad}\left(e^{a} k^{-1}\right)\right)\right|_{\mathfrak{z}^{\perp}(\mathfrak{b})}\right|^{1 / 2}}<\infty .
$$

Definition 5.7. For $\operatorname{Re}(\sigma) \geqslant \sigma_{1}$, set

$$
Z_{\eta, \rho}(\sigma)=\exp \left(-\sum_{\substack{[\gamma] \in\left[\Gamma_{+}\right] \\ \gamma \sim e^{a} k^{-1} \in H}} \frac{\chi_{\mathrm{orb}}\left(B_{[\gamma]} / \mathbb{S}^{1}\right)}{m_{[\gamma]}} \operatorname{Tr}[\rho(\gamma)] \frac{\operatorname{Tr}_{\mathrm{S}}^{E_{\eta}}\left[k^{-1}\right]}{\left.\left|\operatorname{det}\left(1-\operatorname{Ad}\left(e^{a} k^{-1}\right)\right)\right|_{\mathfrak{z}^{\perp}(\mathfrak{b})}\right|^{1 / 2}} e^{-\sigma \ell_{[\gamma]}}\right) .
$$

Recall that by Corollary $3.6, \Lambda^{\bullet}\left(\mathfrak{p}_{\mathfrak{m}, \mathbf{C}}^{*}\right)$ has a unique lift in $R(K)$.

Definition 5.8. Let $\widehat{\eta} \in R(K)$ be the unique virtual representation of $K$ on $E_{\widehat{\eta}}=$ $E_{\hat{\eta}}^{+}-E_{\hat{\eta}}^{-}$such that the following identity in $R\left(K_{M}\right)$ holds,

$$
\left.E_{\widehat{\eta}}\right|_{K_{M}}=\left.\Lambda^{\bullet}\left(\mathfrak{p}_{\mathfrak{m}, \mathbf{C}}^{*}\right) \widehat{\otimes} E_{\eta}\right|_{K_{M}} \in R\left(K_{M}\right) .
$$

Let $C^{\mathfrak{g}, Z, \widehat{\eta}, \rho}$ be the generalised Laplacian acting on $C^{\infty}(Z, \mathscr{F} \hat{\eta} \otimes F)$ introduced after (2.26). For $\lambda \in \mathbf{C}$, set

$$
m_{\eta, \rho}(\lambda)=\operatorname{dim} \operatorname{ker}\left(C^{\mathfrak{g}, Z, \hat{\eta}^{+}, \rho}-\lambda\right)^{N}-\operatorname{dim} \operatorname{ker}\left(C^{\mathfrak{g}, Z, \hat{\eta}^{-}, \rho}-\lambda\right)^{N},
$$

where $N \gg 1$. When $\lambda=0$, set

$$
r_{\eta, \rho}=m_{\eta, \rho}(0)
$$

Let

$$
\operatorname{det}_{\mathrm{gr}}\left(C^{\mathfrak{g}, Z, \widehat{\eta}, \rho}+\sigma\right)=\frac{\operatorname{det}\left(C^{\mathfrak{g}, Z, \hat{\eta}^{+}, \rho}+\sigma\right)}{\operatorname{det}\left(C^{\mathfrak{g}, Z, \widehat{\eta}^{-}, \rho}+\sigma\right)}
$$


be a graded determinant of $C^{\mathfrak{g}, Z, \widehat{\eta}, \rho}+\sigma$. By Theorem 1.5, (5.17) is a meromorphic function on $\sigma \in \mathbf{C}$. Its zeros and poles belong to the set $\left\{-\lambda: \lambda \in \operatorname{Sp}\left(C^{\mathfrak{g}, Z, \widehat{\eta}, \rho}\right)\right\}$. If $\lambda \in \operatorname{Sp}\left(C^{\mathfrak{g}, Z, \widehat{\eta}, \rho}\right)$, the order of the zero at $\sigma=-\lambda$ is $m_{\eta, \rho}(\lambda)$.

Following in [Sh18, (7-60)], set

$$
\sigma_{\eta}=\frac{1}{8} \operatorname{Tr}^{\mathfrak{u}^{\perp}(\mathfrak{b})}\left[C^{\mathfrak{u}(\mathfrak{b}), \mathfrak{u}^{\perp}(\mathfrak{b})}\right]-C^{\mathfrak{u}_{\mathfrak{m}}, \eta} .
$$

Let $P_{\eta}(\sigma)$ be the odd polynomial defined in [Sh18, (7-61)]. When $G$ has non compact centre, we have $\mathfrak{u}^{\perp}(\mathfrak{b})=0$, so

$$
\sigma_{\eta}=-C^{\mathfrak{u}_{\mathfrak{m}}, \eta}
$$

and the polynomial $P_{\eta}$ is given by

$$
P_{\eta}(\sigma)=-\left(\operatorname{dim} E_{\eta}^{+}-\operatorname{dim} E_{\eta}^{-}\right)\left[e\left(T X_{M}, \nabla^{T X_{M}}\right)\right]^{\max } \sigma .
$$

Remark 5.9. In [Sh18, (7-61)], we assume the group $U_{M}$ acts on $\eta$. This action extends to $U(\mathfrak{b})=A_{0} U_{M}$ by requiring $A_{0}$ acts trivially on $\eta$. To define $P_{\eta}(\sigma)$, we use characteristic forms of the homogenous vector bundle $\mathscr{F}_{\mathfrak{b}, \eta}=U \times_{U(\mathfrak{b})} E_{\eta}$ on $U / U(\mathfrak{b})$. In current situation, the $\mathfrak{u}_{\mathfrak{m}}$-action on $E_{\eta}$ does not necessarily lift to $U_{M}$. So the vector bundle $\mathscr{F}_{\mathfrak{b}, \eta}$ is not well defined globally on $U / U(\mathfrak{b})$. However, it is well defined in a neighborhood of $[e] \in U / U(\mathfrak{b})$, so the right-hand sides of [Sh18, (7-8) (7-61)] are still well defined. In particular, $P_{\eta}(\sigma)$ is still well defined.

We have a generalisation of [Sh18, Theorem 7.6].

Theorem 5.10. The Selberg zeta function $Z_{\eta, \rho}(\sigma)$ has a meromorphic extension to $\sigma \in \mathbf{C}$ such that the following identity of meromorphic functions on $\mathbf{C}$ holds,

$$
Z_{\eta, \rho}(\sigma)=\operatorname{det}_{\operatorname{gr}}\left(C^{\mathfrak{g}, Z, \widehat{\eta}, \rho}+\sigma_{\eta}+\sigma^{2}\right) \exp \left(r \operatorname{vol}(Z) P_{\eta}(\sigma)\right) .
$$

The zeros and poles of $Z_{\eta, \rho}(\sigma)$ belong to the set $\left\{ \pm i \sqrt{\lambda+\sigma_{\eta}}: \lambda \in \operatorname{Sp}\left(C^{\mathfrak{g}, Z, \hat{\eta}, \rho}\right)\right\}$. If $\lambda \in \operatorname{Sp}\left(C^{\mathfrak{g}, Z, \hat{\eta}, \rho}\right)$ and $\lambda \neq-\sigma_{\eta}$, the order of the zero at $\sigma= \pm i \sqrt{\lambda+\sigma_{\eta}}$ is $m_{\eta, \rho}(\lambda)$. The order of the zero at $\sigma=0$ is $2 m_{\eta, \rho}\left(-\sigma_{\eta}\right)$. Also,

$$
Z_{\eta, \rho}(\sigma)=Z_{\eta, \rho}(-\sigma) \exp \left(2 r \operatorname{vol}(Z) P_{\eta}(\sigma)\right) .
$$

Proof. For the first part of the theorem, it is enough to show (5.21) for $\sigma \in \mathbf{R}$ large enough, which will be given in Section 5.3. The rest parts of our theorem is a consequence of Theorem 1.5 and (5.21).

\subsection{Proof of (5.21) for $\sigma \gg 1$.}

5.3.1. The case where $\delta(G)=1$ and $Z_{G}$ is non compact. For $\gamma^{\prime} \in M$, let $X_{M}\left(\gamma^{\prime}\right)$ be the symmetric space defined in (2.20) when $G$ and $\gamma$ are replaced by $M$ and $\gamma^{\prime}$. We have a generalisation of [Sh18, Proposition 4.14] and [ShY17, Proposition 5.7].

Proposition 5.11. If $\gamma=e^{a} k^{-1} \in H$ with $a \in \mathfrak{b}$ and $k \in T$, then

$$
\begin{aligned}
& \operatorname{Tr}_{\mathrm{s}}^{[\gamma]}\left[\exp \left(-\frac{t}{2} C^{\mathfrak{g}, X, \hat{\eta}}\right)\right] \\
& \quad=\frac{1}{\sqrt{2 \pi t}} \exp \left(-\frac{|a|^{2}}{2 t}-\frac{t}{2} C^{\mathfrak{u}_{\mathfrak{m}}, \eta}\right)\left[e\left(T X_{M}(k), \nabla^{T X_{M}(k)}\right)\right]^{\max } \operatorname{Tr}_{\mathrm{s}}^{E_{\eta}}\left[k^{-1}\right]
\end{aligned}
$$


If $\gamma$ can not be conjugate into $H$, then

$$
\operatorname{Tr}_{\mathrm{s}}^{[\gamma]}\left[\exp \left(-\frac{t}{2} C^{\mathfrak{g}, X, \hat{\eta}}\right)\right]=0
$$

Proof. Since $K=K_{M} \subset M$ and $\mathfrak{p}=\mathfrak{b} \oplus \mathfrak{p}_{\mathfrak{m}}$, by (5.14), we have

$$
C^{\infty}\left(G, E_{\widehat{\eta}}\right)^{K}=C^{\infty}(\mathbf{R}) \otimes C^{\infty}\left(M, \Lambda^{\cdot}\left(\mathfrak{p}_{\mathfrak{m}, \mathbf{C}}^{*}\right) \widehat{\otimes} E_{\eta \mid K_{M}}\right)^{K_{M}} .
$$

By the identification (5.25), if $\Delta^{\mathbf{R}}$ is the usual Laplacian operator on $\mathbf{R}$, we have

$$
C^{\mathfrak{g}, X, \widehat{\eta}}=-\Delta^{\mathbf{R}}+C^{\mathfrak{m}, X_{M}, \Lambda^{\bullet}\left(\mathfrak{p}_{\mathfrak{m}}^{*}\right) \widehat{\otimes} \eta_{\mid K_{M}} .}
$$

Let $\gamma$ be a semisimple element in $G$. Since $G=\exp (\mathfrak{b}) \times M$, write $\gamma=e^{a} \gamma^{\prime}$ where $a \in \mathfrak{b}$ and $\gamma^{\prime}$ is semisimple in $M$. By (5.26), we have

$$
\operatorname{Tr}_{\mathrm{S}}^{[\gamma]}\left[\exp \left(-\frac{t}{2} C^{\mathfrak{g}, X, \widehat{\eta}}\right)\right]=\operatorname{Tr}^{\left[e^{a}\right]}\left[\exp \left(\frac{t}{2} \Delta^{\mathbf{R}}\right)\right] \operatorname{Tr}_{\mathrm{S}}^{\left[\gamma^{\prime}\right]}\left[\exp \left(-\frac{t}{2} C^{\mathfrak{m}, X_{M}, \Lambda^{\bullet}\left(\mathfrak{p}_{\mathfrak{m}}^{*}\right) \widehat{\otimes} \eta_{\mid K_{M}}}\right)\right]
$$

Clearly, we have

$$
\operatorname{Tr}^{\left[e^{a}\right]}\left[\exp \left(\frac{t}{2} \Delta^{\mathbf{R}}\right)\right]=\frac{1}{\sqrt{2 \pi t}} e^{-\frac{|a|^{2}}{2 t}}
$$

If $\gamma$ can not be conjugate in $H$, then $\gamma^{\prime}$ is not elliptic in $M$, by [Sh18, (8-82)], we have

$$
\operatorname{Tr}_{\mathrm{s}}^{\left[\gamma^{\prime}\right]}\left[\exp \left(-\frac{t}{2} C^{\left.\mathfrak{m}, X_{M}, \Lambda^{\bullet}\left(\mathfrak{p}_{\mathfrak{m}}^{*}\right) \widehat{\otimes} \eta_{\mid K_{M}}\right)}\right]=0\right.
$$

If $\gamma \in H$, then $\gamma^{\prime}=k^{-1} \in T$. By [Sh18, (8-83)], we have

$$
\begin{aligned}
\operatorname{Tr}_{\mathrm{s}}^{\left[k^{-1}\right]}\left[\exp \left(-\frac{t}{2} C^{\left.\mathfrak{m}, X_{M}, \Lambda^{\bullet}\left(\mathfrak{p}_{\mathfrak{m}}^{*}\right) \widehat{\otimes} \eta_{\mid K_{M}}\right)}\right]\right. \\
=\left[e\left(T X_{M}(k), \nabla^{T X_{M}(k)}\right)\right]^{\max } \operatorname{Tr}_{\mathrm{s}}^{E_{\eta}}\left[k^{-1}\right] \exp \left(-\frac{t}{2} C^{\mathfrak{u}_{\mathfrak{m}}, \eta}\right)
\end{aligned}
$$

By (5.27)-(5.30), we get (5.23) and (5.24).

Proceeding as in the proof of [Sh18, Theorem 5.6], using Theorem 2.8 and Proposition 5.11 instead of [Sh18, Theorem 4.10 and Proposition 4.14], we get (5.21) for $\sigma \gg 1$.

5.3.2. The case where $\delta(G)=1$ and $Z_{G}$ is compact. Although, Assumption 5.4 on $\eta$ is different from [Sh18, Assumption 7.1], by Remarks 5.6 and 5.9, the statement of [Sh18, Theorem 7.3] still holds. Therefore, the proof of (5.21) for $\sigma \gg 1$ is identical to the one given in [Sh18, Theorem 7.6], except that we use Theorem 2.8 instead of [Sh18, Theorem 4.10].

\subsection{Proof of Theorem 4.3.}


5.4.1. The case where $\delta(G)=1$ and $Z_{G}$ is non compact. Since $G$ has a non compact centre, by the second equation of (5.2), the denominator $\left.\left|\operatorname{det}\left(1-\operatorname{Ad}\left(e^{a} k^{-1}\right)\right)\right|_{\mathfrak{z}^{\perp}(\mathfrak{b})}\right|^{1 / 2}$ in (5.13) disappears. When $\eta=\mathbf{1}$ is the trivial representation, by (4.5), (5.13), (5.14), and (5.19), we have

$$
R_{\rho}(\sigma)=\left(Z_{\mathbf{1}, \rho}(\sigma)\right)^{-1}, \quad E_{\widehat{\mathbf{1}}}=\sum_{i=1}^{m}(-1)^{i-1} i \Lambda^{i}\left(\mathfrak{p}_{\mathbf{C}}^{*}\right) \in R(K), \quad \sigma_{\mathbf{1}}=0 .
$$

By Theorem 5.10, and by (1.70) and (5.31), we see that $R_{\rho}(\sigma)$ has a meromorphic extension and

$$
R_{\rho}(\sigma)=\exp \left(r \operatorname{vol}(Z)\left[e\left(T X_{M}, \nabla^{T X_{M}}\right)\right]^{\max } \sigma\right) T_{\rho}\left(\sigma^{2}\right) .
$$

Set

$$
C_{\rho}=1, \quad r_{\rho}=2 \chi_{\mathrm{CM}}^{\prime}(F) .
$$

By (1.71), (5.32), and (5.33), we get (4.6). By Proposition 1.12, (1.69), and (5.33), we see that (4.7) holds with $k=0$. The proof of Theorem 4.3 is complete when $\delta(G)=1$ and $Z_{G}$ is not compact.

5.4.2. The case where $\delta(G)=1$ and $Z_{G}$ is compact. For $0 \leqslant j \leqslant 2 \ell$, let $\eta_{j}$ be the representation of $M$ on $\Lambda^{j}\left(\mathfrak{n}_{\mathbf{C}}^{*}\right)$. By Corollary 3.6 (or [Sh18, Corollary 6.12]) and [Sh18, Proposition 6.13], $\eta_{j}$ satisfies Assumption 5.4. By Theorem 5.10, we see that $Z_{\eta_{j}, \rho}$ has a meromorphic extension to C. Using the relation ([Sh18, (7-65)])

$$
R_{\rho}(\sigma)=\prod_{j=0}^{2 \ell} Z_{\eta_{j}, \rho}\left(\sigma+(j-\ell)\left|\alpha_{0}\right|\right)^{(-1)^{j-1}}
$$

we see that $R_{\rho}(\sigma)$ has a meromorphic extension.

Write $r_{j}=r_{\eta_{j}, \rho}$. As in [Sh18, (7-74),(7-75)], set

$$
C_{\rho}=\prod_{j=0}^{\ell-1}\left(-4(\ell-j)^{2}\left|\alpha_{0}\right|^{2}\right)^{(-1)^{j-1} r_{j}}, \quad r_{\rho}=2 \sum_{j=0}^{\ell}(-1)^{j-1} r_{j} .
$$

Proceeding exactly as in [Sh18, (7-71)-(7-78)], we get (4.6). The proof of (4.7) will be given in Section 6.3.

Remark 5.12. Note that $r_{\rho}$ is even. By (5.35) and by [Sh18, (7-74), (7-80)], we have

$$
\begin{aligned}
& C_{\rho}=(-1)^{\chi_{\mathrm{CM}}^{\prime}(X, F)+\frac{r_{\rho}}{2}} \prod_{j=0}^{\ell-1}\left(4(\ell-j)^{2}\left|\alpha_{0}\right|^{2}\right)^{(-1)^{j-1} r_{j}}, \\
& r_{\rho}=\chi_{\mathrm{CM}}^{\prime}(Z, F)+(-1)^{\ell-1} r_{\ell}=2 \chi_{\mathrm{CM}}^{\prime}(Z, F)+2 \sum_{j=0}^{\ell-1}(-1)^{j} r_{j} .
\end{aligned}
$$

\section{A COHOMOLOGICAL FORMULA FOR $r_{j}$}

The purpose of this section is to establish (4.7) when $\delta(G)=1$ and $Z_{G}$ is compact. Its proof relies on some results from the representation theory of real reductive groups.

This section is organised as follows. In Section 6.1, we recall the definition of infinitesimal characters of $\mathfrak{g}_{\mathbf{C}}$-modules and some basic properties of the HarishChandra $\left(\mathfrak{g}_{\mathbf{c}}, K\right)$-modules. 
In Section 6.2, we study the right regular representation of $G$ on $C^{\infty}\left(\Gamma \backslash G, \widehat{p}^{*} F\right)$. Given a character $\chi: \mathscr{Z}\left(\mathfrak{g}_{\mathbf{C}}\right) \rightarrow \mathbf{C}$, we introduce a Harish-Chandra $\left(\mathfrak{g}_{\mathbf{C}}, K\right)$-modules $V_{\chi}(\Gamma, \rho) \subset C^{\infty}\left(\Gamma \backslash G, \hat{p}^{*} F\right)$ with generalised infinitesimal character $\chi$.

Finally, in Section 6.3, assuming that $\delta(G)=1$ and $Z_{G}$ is compact, we write $r_{j}$ as a sum of Euler characteristic numbers of certain cohomologies of $V_{\chi_{1}}(\Gamma, \rho)$, where $\chi_{\mathbf{1}}$ is the trivial character. We establish (4.7).

In Sections 6.1 and 6.2, we assume neither $\delta(G)=1$ nor $Z_{G}$ is compact.

6.1. Preliminary on the representation theory. Recall that $G$ is a linear connected real reductive group. We use the notation in Sections 2.1 and 2.2. Recall that $\mathscr{Z}\left(\mathfrak{g}_{\mathbf{C}}\right)$ is the centre of the enveloping algebra of $\mathfrak{g}_{\mathbf{C}}$.

A morphism of algebras $\chi: \mathscr{Z}\left(\mathfrak{g}_{\mathbf{C}}\right) \rightarrow \mathbf{C}$ will be called a character of $\mathscr{Z}\left(\mathfrak{g}_{\mathbf{C}}\right)$. Clearly, for $a \in \mathbf{C}$, we have

$$
\chi(a)=a .
$$

Definition 6.1. A complex representation of $\mathfrak{g}_{\mathbf{C}}$ is said to have an infinitesimal character $\chi$ if $z \in \mathscr{Z}\left(\mathfrak{g}_{\mathbf{C}}\right)$ acts as a scalar $\chi(z) \in \mathbf{C}$.

A complex representation of $\mathfrak{g}_{\mathbf{C}}$ is said to have a generalised infinitesimal character $\chi$ if there is $i \gg 1$ such that for all $z \in \mathscr{Z}\left(\mathfrak{g}_{\mathbf{C}}\right),(z-\chi(z))^{i}$ acts like 0 .

The infinitesimal character of a trivial representation will be called the trivial character $\chi_{\mathbf{1}}$. Note that in [Sh18, Section 8], such a character is denoted by 0 . We will not use this notation here.

Definition 6.2. A complex $\mathscr{U}\left(\mathfrak{g}_{\mathbf{C}}\right)$-module $V$, equipped with an action of $K$, is called a $\left(\mathfrak{g}_{\mathbf{C}}, K\right)$-module, if

(1) every $v \in V$ is $K$-finite, i.e., $\{k \cdot v\}_{k \in K}$ spans a finite dimensional vector space;

(2) the actions of $\mathfrak{g}_{\mathbf{C}}$ and $K$ are compatible.

A $\left(\mathfrak{g}_{\mathbf{C}}, K\right)$-module $V$ is called a Harish-Chandra $\left(\mathfrak{g}_{\mathbf{C}}, K\right)$-module, if

(1) the space $V$ is finitely generated as a $\mathscr{U}\left(\mathfrak{g}_{\mathbf{C}}\right)$-module;

(2) each irreducible $K$-module occurs only for a finite number of times in $V$.

Let $V$ be a Harish-Chandra $\left(\mathfrak{g}_{\mathbf{C}}, K\right)$-module. By [K86, Proposition 10.43], submodules or quotient modules of $V$ is still a Harish-Chandra $\left(\mathfrak{g}_{\mathbf{C}}, K\right)$-module. When $V$ is irreducible, by [K86, Corollary 8.13], $V$ has an infinitesimal character. For general $V$, by [K86, Proposition 10.41], there are finitely many Harish-Chandra $\left(\mathfrak{g}_{\mathbf{C}}, K\right)$-submodules $V_{\chi} \subset V$ of $V$ with generalised infinitesimal character $\chi$, so that

$$
V=\bigoplus_{\chi} V_{\chi}
$$

By [K86, Corollary 10.42], $V$ has a finite composition series, i.e., there exist finitely many Harish-Chandra $\left(\mathfrak{g}_{\mathbf{C}}, K\right)$-submodules

$$
V=V_{N_{0}} \supset V_{N_{0}-1} \supset \cdots \supset V_{0} \supset V_{-1}=0,
$$

such that each quotient $V_{s} / V_{s-1}$ with $0 \leqslant s \leqslant N_{0}$ is irreducible. Moreover, the length $N_{0} \in \mathbf{N}$, the set of all irreducible quotients and their multiplicities are the 
same for all the composition series. Also, if $E$ is a finite dimensional representation of $K$, then

$$
\operatorname{dim}(V \otimes E)^{K}=\sum_{s=0}^{N_{0}} \operatorname{dim}\left(\left(V_{s} / V_{s-1}\right) \otimes E\right)^{K} .
$$

Proposition 6.3. Given a character $\chi: \mathscr{Z}\left(\mathfrak{g}_{\mathbf{C}}\right) \rightarrow \mathbf{C}$, there is a finite dimensional representation $E$ of $K$ such that if $V$ is a Harish-Chandra $\left(\mathfrak{g}_{\mathbf{c}}, K\right)$-module with generalised infinitesimal character $\chi$, then

$$
V=0 \Longleftrightarrow(V \otimes E)^{K}=0 .
$$

Proof. It is enough to show the inverse direction. By a fundamental theorem of Harish-Chandra [K86, Corollary 10.37], there are only finitely many irreducible Harish-Chandra $\left(\mathfrak{g}_{\mathbf{C}}, K\right)$-modules $V_{1}, \ldots, V_{k}$ with infinitesimal character $\chi$. Let $E_{i}$ be a $K$-type of $V_{i}$. Set

$$
E=\bigoplus_{i=1}^{k} E_{i}^{*}
$$

By (6.6), for all $1 \leqslant i \leqslant k$,

$$
\left(V_{i} \otimes E\right)^{K} \neq 0 .
$$

By (6.4) and (6.7), we get the inverse direction in (6.5).

6.2. The right regular representation on $C^{\infty}\left(\Gamma \backslash G, \widehat{p}^{*} F\right)$. Recall that $\Gamma \subset G$ is a discrete cocompact and torsion free subgroup of $G$ and that $\rho: \Gamma \rightarrow \mathrm{GL}_{r}(\mathbf{C})$ is a representation of $\Gamma$. Recall also that $F$ is the flat vector bundle on $Z=\Gamma \backslash G / K$ defined in (1.33). We fix a Hermitian metric $g^{F}$ on $F$. Note that the choice of $g^{F}$ is irrelevant.

Let $g^{\widehat{p}^{*} F}$ be the pull back metric on $\widehat{p}^{*} F$. Let $L^{2}\left(\Gamma \backslash G, \widehat{p}^{*} F\right)$ be the $L^{2}$-space associated to $g^{\hat{p}^{*} F}$ and to an invariant measure on $\Gamma \backslash G$. The group $G$ acts on the right on $L^{2}\left(\Gamma \backslash G, \hat{p}^{*} F\right)$. Since $\rho$ is not necessarily unitary, this $G$-action is not always unitary. Since $g^{\widehat{p}^{*} F}$ is $K$-invariant, the above $G$-action restricts to a unitary representation of $K$. Let $\widehat{K}$ be the set of equivalent classes of irreducible representations of $K$. If $\tau \in \widehat{K}$, let $L_{\tau}^{2}\left(\Gamma \backslash G, \widehat{p}^{*} F\right)$ be the $\tau$-isotropic subspace of $L^{2}\left(\Gamma \backslash G, \widehat{p}^{*} F\right)$. By (2.16) and (2.25), we have

$$
L_{\tau}^{2}\left(\Gamma \backslash G, \widehat{p}^{*} F\right) \simeq L^{2}\left(Z, \mathscr{F}_{\tau}^{*} \otimes F\right) \otimes E_{\tau} .
$$

By Peter-Weyl's theorem, we have a direct sum of Hilbert spaces

$$
L^{2}\left(\Gamma \backslash G, \widehat{p}^{*} F\right)=\bigoplus_{\tau \in \widehat{K}}^{\text {Hil }} L_{\tau}^{2}\left(\Gamma \backslash G, \widehat{p}^{*} F\right) .
$$

By (6.8), the Casimir operator $C^{\mathfrak{g}, Z, \tau^{*}, \rho}$ defines an unbounded operator on the Hilbert space $L_{\tau}^{2}\left(\Gamma \backslash G, \hat{p}^{*} F\right)$. If $\lambda \in \operatorname{Sp}\left(C^{\mathfrak{g}, Z, \tau^{*}, \rho}\right)$, as in (1.7), let $C_{\tau, \lambda}^{\infty}\left(\Gamma \backslash G, \hat{p}^{*} F\right)$ be the corresponding finite dimensional characteristic space. As the notation indicates,

$$
C_{\tau, \lambda}^{\infty}\left(\Gamma \backslash G, \widehat{p}^{*} F\right) \subset C^{\infty}\left(\Gamma \backslash G, \widehat{p}^{*} F\right)
$$


Let $V(\Gamma, \rho)$ be the subspace of $C^{\infty}\left(\Gamma \backslash G, \hat{p}^{*} F\right)$ defined by the algebraic sum

$$
V(\Gamma, \rho)=\bigoplus_{\tau \in \widehat{K}} \bigoplus_{\lambda \in \operatorname{Sp}\left(C^{\mathfrak{g}}, Z, \tau^{*}, \rho\right)} C_{\tau, \lambda}^{\infty}\left(\Gamma \backslash G, \hat{p}^{*} F\right) .
$$

Clearly, the group $K$ acts on $V(\Gamma, \rho)$ and the elements of $V(\Gamma, \rho)$ are $K$-finite.

By (1.9), (6.9), and (6.11), we have

$$
L^{2}\left(\Gamma \backslash G, \widehat{p}^{*} F\right)=\overline{V(\Gamma, \rho)} .
$$

Proposition 6.4. The algebra $\mathscr{U}\left(\mathfrak{g}_{\mathbf{C}}\right)$ acts on $V(\Gamma, \rho)$, so that $V(\Gamma, \rho)$ is a $\left(\mathfrak{g}_{\mathbf{C}}, K\right)$ module.

Proof. We have seen in the arguments below (6.11) that elements of $V(\Gamma, \rho)$ are $K$-finite. We need to show that $\mathfrak{g}_{\mathbf{C}}$ acts on $V(\Gamma, \rho)$, or equivalently, for any pair $(\tau, \lambda)$,

$$
\mathfrak{g} \cdot C_{\tau, \lambda}^{\infty}\left(\Gamma \backslash G, \widehat{p}^{*} F\right) \subset V(\Gamma, \rho) .
$$

Let us follow the proof of [K86, Proposition 8.5]. Since the space $C_{\tau, \lambda}^{\infty}\left(\Gamma \backslash G, \hat{p}^{*} F\right) \subset$ $C^{\infty}\left(\Gamma \backslash G, \widehat{p}^{*} F\right)$ is $K$-invariant, for $k \in K$ and $a \in \mathfrak{g}$, we have

(6.14) $k \cdot a \cdot C_{\tau, \lambda}^{\infty}\left(\Gamma \backslash G, \hat{p}^{*} F\right) \subset(\operatorname{Ad}(k) a) \cdot C_{\tau, \lambda}^{\infty}\left(\Gamma \backslash G, \widehat{p}^{*} F\right) \subset \mathfrak{g} \cdot C_{\tau, \lambda}^{\infty}\left(\Gamma \backslash G, \hat{p}^{*} F\right)$.

By (6.14), the finite dimensional space $\mathfrak{g} \cdot C_{\tau, \lambda}^{\infty}\left(\Gamma \backslash G, \hat{p}^{*} F\right)$ is $K$-invariant. In particular, the elements of $\mathfrak{g} \cdot C_{\tau, \lambda}^{\infty}\left(\Gamma \backslash G, \widehat{p}^{*} F\right)$ are $K$-finite. Therefore,

$$
\mathfrak{g} \cdot C_{\tau, \lambda}^{\infty}\left(\Gamma \backslash G, \widehat{p}^{*} F\right) \subset \bigoplus_{\tau \in \widehat{K}} C_{\tau}^{\infty}\left(\Gamma \backslash G, \widehat{p}^{*} F\right) .
$$

Since the Casimir commutes with the $\mathfrak{g}$-actions, by (6.15), we have

$$
\mathfrak{g} \cdot C_{\tau, \lambda}^{\infty}\left(\Gamma \backslash G, \widehat{p}^{*} F\right) \subset \bigoplus_{\tau \in \widehat{K}} C_{\tau, \lambda}^{\infty}\left(\Gamma \backslash G, \widehat{p}^{*} F\right) .
$$

By (6.11) and (6.16), we get (6.13).

Note that $\mathscr{Z}\left(\mathfrak{g}_{\mathbf{C}}\right)$ acts on the finite dimensional space $C_{\tau, \lambda}^{\infty}\left(\Gamma \backslash G, \widehat{p}^{*} F\right)$. For a character $\chi$ of $\mathscr{Z}\left(\mathfrak{g}_{\mathbf{C}}\right)$, set

$$
V_{\chi}(\Gamma, \rho)=\left\{v \in V(\Gamma, \rho): \exists i \in \mathbf{N}, \forall z \in \mathscr{Z}\left(\mathfrak{g}_{\mathbf{C}}\right),(z-\chi(z))^{i} v=0\right\} .
$$

By (6.11) and (6.17), we have an infinite algebraic sum

$$
V(\Gamma, \rho)=\bigoplus_{\chi} V_{\chi}(\Gamma, \rho) .
$$

By Proposition $6.4, V_{\chi}(\Gamma, \rho)$ is a $\left(\mathfrak{g}_{\mathbf{C}}, K\right)$-module.

Proposition 6.5. Each $V_{\chi}(\Gamma, \rho)$ is a Harish-Chandra $\left(\mathfrak{g}_{\mathbf{C}}, K\right)$-module.

Proof. By (6.17), we have

$$
V_{\chi}(\Gamma, \rho) \subset \bigoplus_{\tau \in \widehat{K}} C_{\tau, \chi(C)}^{\infty}\left(\Gamma \backslash G, \widehat{p}^{*} F\right) .
$$

In particular, the multiplicity of each $K$-type in $V_{\chi}(\Gamma, \rho)$ is finite.

It remains to show that $V_{\chi}(\Gamma, \rho)$ is a finitely generated $\mathscr{U}\left(\mathfrak{g}_{\mathbf{C}}\right)$-module. Otherwise, we can find a sequence $\left(v_{n}\right)_{n \in \mathbf{N}}$ in $V_{\chi}(\Gamma, \rho)$ such that

$$
0 \varsubsetneqq \mathscr{U}\left(\mathfrak{g}_{\mathbf{C}}\right) v_{1} \varsubsetneqq \ldots \varsubsetneqq \sum_{i=1}^{n-1} \mathscr{U}\left(\mathfrak{g}_{\mathbf{C}}\right) v_{i} \varsubsetneqq \sum_{i=1}^{n} \mathscr{U}\left(\mathfrak{g}_{\mathbf{C}}\right) v_{i} \varsubsetneqq \ldots
$$


For $n \geqslant 1, \sum_{i=1}^{n} \mathscr{U}\left(\mathfrak{g}_{\mathbf{C}}\right) v_{i}$ is a Harish-Chandra $\left(\mathfrak{g}_{\mathbf{C}}, K\right)$-module. Since there are only finitely many irreducible Harish-Chandra $\left(\mathfrak{g}_{\mathbf{C}}, K\right)$-modules with infinitesimal character $\chi$ ([K86, Corollary 10.37]), there is at least one, denoted by $V$, occurs an infinite number of times in $\sum_{i=1}^{n} \mathscr{U}\left(\mathfrak{g}_{\mathbf{C}}\right) v_{i} / \sum_{i=1}^{n-1} \mathscr{U}\left(\mathfrak{g}_{\mathbf{C}}\right) v_{i}$ with $n \geqslant 1$. If $\tau \in \widehat{K}$ is a $K$ type in $V$, then $\tau$ occurs infinitely many times in $V_{\chi}(\Gamma, \rho)$, which is in contradiction with (6.19).

The following proposition is an immediate consequence of (6.11) and (6.17).

Proposition 6.6. If $\left(\tau, E_{\tau}\right)$ is a representation of $K$, then we have a finite sum

$$
C_{\lambda}^{\infty}\left(Z, \mathscr{F}_{\tau} \otimes F\right)=\bigoplus_{\chi: \chi\left(C^{\mathfrak{g}}\right)=\lambda}\left(V_{\chi}(\Gamma, \rho) \otimes E_{\tau}\right)^{K} .
$$

We use the notation in Section 1.6. Recall that $\operatorname{Rep}\left(\Gamma, \mathbf{C}^{r}\right)$ is the set of all $r$ dimensional representations of $\Gamma$.

Proposition 6.7. There is $k_{0} \in \mathbf{N}$ such that if $\chi$ is a character of $\mathscr{Z}\left(\mathfrak{g}_{\mathbf{C}}\right)$ and if $\rho_{0} \in$ $\operatorname{Rep}\left(\Gamma, \mathbf{C}^{r}\right)$ is such that $V_{\chi}\left(\Gamma, \rho_{0}\right)=0$, then there is $\epsilon>0$ such that if $\rho \in \operatorname{Rep}\left(\Gamma, \mathbf{C}^{r}\right)$ is $C^{k_{0}} \epsilon$-close to $\rho_{0}$, then

$$
V_{\chi}(\Gamma, \rho)=0 .
$$

Proof. We fix a character $\chi$ of $\mathscr{Z}\left(\mathfrak{g}_{\mathbf{C}}\right)$. Let $E_{\tau}$ be the $K$-representation constructed in Proposition 6.3, so that (6.22) is equivalent to

$$
\left(V_{\chi}(\Gamma, \rho) \otimes E_{\tau}\right)^{K}=0
$$

By [K86, Theorem 8.19], $\mathscr{Z}\left(\mathfrak{g}_{\mathbf{C}}\right)$ is a polynomial algebra of dim $\mathfrak{h}$ variables. Let $z_{1}=C^{\mathfrak{g}}, z_{2}, \ldots, z_{\operatorname{dimh}}$ be a set of generators. If $z \in \mathscr{Z}\left(\mathfrak{g}_{\mathbf{C}}\right)$, denote by $z^{Z, \tau, \rho}$ the action of $z$ on $C^{\infty}\left(Z, \mathscr{F}_{\tau} \otimes F_{\rho}\right)$. Clearly, $z^{Z, \tau, \rho}$ is a differential operator. Recall that we have choosen a Hermitian metric on $F_{\rho}$ and it induces an $L^{2}$-product on $C^{\infty}\left(Z, \mathscr{F}_{\tau} \otimes F_{\rho}\right)$. Take $d \in \mathbf{N}$ such that $2 d$ is strictly larger than the order of the differential operators $z_{i}^{Z, \tau, \rho}$ with $2 \leqslant i \leqslant \operatorname{dimh}$. Set

$$
\begin{aligned}
L_{\rho}=\left\{( C ^ { \mathfrak { g } , Z , \tau , \rho } - \chi ( C ^ { \mathfrak { g } } ) ) ^ { * } \left(C^{\mathfrak{g}, \tau, E, \rho}-\right.\right. & \left.\left.\chi\left(C^{\mathfrak{g}}\right)\right)\right\}^{d} \\
& +\sum_{i=2}^{\operatorname{dim} \mathfrak{h}}\left(z_{i}^{Z, \tau, \rho}-\chi\left(z_{i}\right)\right)^{*}\left(z_{i}^{Z, \tau, \rho}-\chi\left(z_{i}\right)\right),
\end{aligned}
$$

where $^{*}$ is the adjoint with respect to the $L^{2}$-product. Then $L_{\rho}$ is a self-adjoint elliptic differential operator of order $4 d$.

We claim that

$$
\left(V_{\chi}(\Gamma, \rho) \otimes E_{\tau}\right)^{K}=0 \Longleftrightarrow \operatorname{ker} L_{\rho}=0 .
$$

Indeed, by (6.24), we have

$$
\operatorname{ker} L_{\rho}=\bigcap_{i=1}^{\operatorname{dim} \mathfrak{h}} \operatorname{ker}\left(z_{i}^{Z, \tau, \rho}-\chi\left(z_{i}\right)\right) \subset\left(V_{\chi}(\Gamma, \rho) \otimes E_{\tau}\right)^{K},
$$

which implies the direction $\Longrightarrow$. For the other direction, if $\left(V_{\chi}(\Gamma, \rho) \otimes E_{\tau}\right)^{K} \neq 0$, since the operators $z_{i}^{Z, \tau, \rho}$ commute and act on the finite dimensional vector space $\left(V_{\chi}(\Gamma, \rho) \otimes E_{\tau}\right)^{K}$, they have a common eigenvector. This means $\operatorname{ker} L_{\rho} \neq 0$. 
Fix now $\rho_{0} \in \operatorname{Rep}\left(\Gamma, \mathbf{C}^{r}\right)$ such that $V_{\chi}\left(\Gamma, \rho_{0}\right)=0$. By (6.25), $L_{\rho_{0}}$ is invertible. As in (1.76), if $\rho$ is in a small $C^{0}$-neighborhood of $\rho_{0}$, we can identity $\left(F_{\rho}, \nabla^{F_{\rho}}\right)$ with $\left(F_{\rho_{0}}, \nabla^{F_{\rho_{0}}}+A_{\rho}\right)$. We take $g^{F_{\rho}}=g^{F_{\rho_{0}}}$. Then, $L_{\rho}$ and $L_{\rho_{0}}$ act on the same space $C^{\infty}\left(Z, \mathscr{F}_{\tau} \otimes F_{\rho_{0}}\right)$ with the same principal symbol. Set

$$
B_{\rho}=L_{\rho}-L_{\rho_{0}} .
$$

Then, $B_{\rho}$ is a differential operator of order $4 d-1$. By (1.77), there is $C>0$ such that

$$
\left\|B_{\rho}\right\|_{\mathscr{H}^{4 d-1}, L^{2}} \leqslant C\left\|A_{\rho}\right\|_{C^{4 d-1}} .
$$

By (6.27), since $L_{\rho_{0}}$ is invertible, we have

$$
L_{\rho}=\left(1+B_{\rho} L_{\rho_{0}}^{-1}\right) L_{\rho_{0}} .
$$

Take $k_{0}=4 d-1$. By (6.28), if $\left\|A_{\rho}\right\|_{C^{k_{0}}}$ is small enough, then $\left\|B_{\rho} L_{\rho_{0}}^{-1}\right\|_{L^{2}, L^{2}}<1$ and $L_{\rho}$ is invertible. By (6.25), we get (6.23).

We have an analogue [Sh18, (8-68)].

Theorem 6.8. Let $\rho_{0} \in \operatorname{Rep}\left(\Gamma, \mathbf{C}^{r}\right)$ be an acyclic and unitary representation of $\Gamma$. There is $\epsilon>0$ such that if $\rho$ is $C^{k_{0}} \epsilon$-close to $\rho_{0}$, then

$$
V_{\chi_{1}}(\Gamma, \rho)=0 .
$$

Proof. In [Sh18, Proposition 8.12], using fundamental results of Vogan-Zuckerman [VoZu84], Vogan [Vo84], and Salamanca-Riba [SR99], we have shown that $\rho_{0}$ is unitary and acyclic, if and only if

$$
V_{\chi_{1}}\left(\Gamma, \rho_{0}\right)=0
$$

By Proposition 6.7, if $\rho$ is close enough to $\rho_{0}$ in the sense of $C^{k_{0}}$, we get (6.30).

6.3. Formulas for $r_{\eta, \rho}$ and $r_{j}$. Assume now $\delta(G)=1$ and $Z_{G}$ is compact. Let $\rho: \Gamma \rightarrow \mathrm{GL}_{r}(\mathbf{C})$ be a representation of $\Gamma$, and let $\eta$ be a virtual representation of $M$ satisfying Assumption 5.4. Recall that $\widehat{\eta}$ is defined in (5.14).

If $V$ is a Harish-Chandra $\left(\mathfrak{g}_{\mathbf{C}}, K\right)$-module, denote by $H^{\bullet}(\mathfrak{g}, K ; V)$ the associated $\left(\mathfrak{g}_{\mathbf{C}}, K\right)$-cohomology and by $H_{\bullet}(\mathfrak{n}, V)$ the $\mathfrak{n}$-homology. By [HeSc83, Proposition 2.24], $H_{\bullet}(\mathfrak{n}, V)$ is a Harish-Chandra $\left(\mathfrak{m}_{\mathbf{C}}, K_{M}\right)$-module. Let $\chi\left(K / K_{M}\right)$ be the Euler characteristic number of $K / K_{M}$.

Proposition 6.9. The following identity holds,

$$
\begin{aligned}
r_{\eta, \rho}=\frac{1}{\chi\left(K / K_{M}\right)} \sum_{\substack{\chi: \chi\left(C^{\mathfrak{g}}\right)=0 \\
0 \leqslant i \leqslant \operatorname{dim} \mathfrak{p}_{\mathfrak{m}} \\
0 \leqslant k \leqslant 2 \ell}}(-1)^{i+k}\{ & \operatorname{dim} H^{i}\left(\mathfrak{m}, K_{M} ; H_{k}\left(\mathfrak{n}, V_{\chi}(\Gamma, \rho)\right) \otimes E_{\eta}^{+}\right) \\
& \left.-\operatorname{dim} H^{i}\left(\mathfrak{m}, K_{M} ; H_{k}\left(\mathfrak{n}, V_{\chi}(\Gamma, \rho)\right) \otimes E_{\eta}^{-}\right)\right\},
\end{aligned}
$$

where the above sum is finite. 
Proof. We claim that it is enough to show that

(6.33) $\operatorname{dim}\left(V_{\chi}(\Gamma, \rho) \otimes E_{\widehat{\eta}}^{+}\right)^{K}-\operatorname{dim}\left(V_{\chi}(\Gamma, \rho) \otimes E_{\widehat{\eta}}^{-}\right)^{K}$

$$
\begin{aligned}
=\frac{1}{\chi\left(K / K_{M}\right)} \sum_{\substack{0 \leqslant i \leqslant \operatorname{dim} \mathfrak{p}_{\mathfrak{m}} \\
0 \leqslant k \leqslant 2 \ell}}(-1)^{i+k}\left\{\operatorname{dim}\left(\Lambda^{i}\left(\mathfrak{p}_{\mathfrak{m}}^{*}\right) \otimes H_{k}\left(\mathfrak{n}, V_{\chi}(\Gamma, \rho)\right) \otimes E_{\eta}^{+}\right)^{K_{M}}\right. \\
\left.-\operatorname{dim}\left(\Lambda^{i}\left(\mathfrak{p}_{\mathfrak{m}}^{*}\right) \otimes H_{k}\left(\mathfrak{n}, V_{\chi}(\Gamma, \rho)\right) \otimes E_{\eta}^{-}\right)^{K_{M}}\right\} .
\end{aligned}
$$

Indeed, by (5.16) and (6.21), we have a finite sum

$$
r_{\eta, \rho}=\sum_{\chi: \chi\left(C^{\mathfrak{g}}\right)=0}\left\{\operatorname{dim}\left(V_{\chi}(\Gamma, \rho) \otimes E_{\widehat{\eta}}^{+}\right)^{K}-\operatorname{dim}\left(V_{\chi}(\Gamma, \rho) \otimes E_{\widehat{\eta}}^{-}\right)^{K}\right\} .
$$

Equation (6.32) follows from (6.33), (6.34), and the Euler formula [Sh18, (8-85)].

If $V_{\chi}(\Gamma, \rho)$ is unitary and irreducible, then (6.33) is just [Sh18, Theorem 8.14]. However, the proof extends to any irreducible $\left(\mathfrak{g}_{\mathbf{C}}, K\right)$-module without any change. In particular, if $V_{\chi}(\Gamma, \rho)$ has a composition series (6.3), for $0 \leqslant s \leqslant N_{0}$, we have

$$
\begin{aligned}
& \operatorname{dim}\left(\left(V_{s} / V_{s-1}\right) \otimes E_{\widehat{\eta}}^{+}\right)^{K}-\operatorname{dim}\left(\left(V_{s} / V_{s-1}\right) \otimes E_{\widehat{\eta}}^{-}\right)^{K} \\
& =\frac{1}{\chi\left(K / K_{M}\right)} \sum_{\substack{0 \leq i \leq \operatorname{dim} \mathfrak{p}_{\mathfrak{m}} \\
0 \leqslant k \leqslant 2 \ell}}(-1)^{i+k}\left\{\operatorname{dim}\left(\Lambda^{i}\left(\mathfrak{p}_{\mathfrak{m}}^{*}\right) \otimes H_{k}\left(\mathfrak{n}, V_{s} / V_{s-1}\right) \otimes E_{\eta}^{+}\right)^{K_{M}}\right. \\
& \left.\quad-\operatorname{dim}\left(\Lambda^{i}\left(\mathfrak{p}_{\mathfrak{m}}^{*}\right) \otimes H_{k}\left(\mathfrak{n}, V_{s} / V_{s-1}\right) \otimes E_{\eta}^{-}\right)^{K_{M}}\right\}
\end{aligned}
$$

Using the exact sequence of the Harish-Chandra $\left(\mathfrak{g}_{\mathbf{C}}, K\right)$-modules,

$$
0 \rightarrow V_{s-1} \rightarrow V_{s} \rightarrow V_{s} / V_{s-1} \rightarrow 0
$$

we have

$$
\operatorname{dim}\left(\left(V_{s} / V_{s-1}\right) \otimes E_{\hat{\eta}}^{ \pm}\right)^{K}=\operatorname{dim}\left(V_{s} \otimes E_{\hat{\eta}}^{ \pm}\right)^{K}-\operatorname{dim}\left(V_{s-1} \otimes E_{\hat{\eta}}^{ \pm}\right)^{K} .
$$

On the other hand, by (6.36), we have the long exact sequence of HarishChandra $\left(\mathfrak{m}_{\mathbf{C}}, K_{M}\right)$-modules,

$$
\cdots \rightarrow H_{k}\left(\mathfrak{n}, V_{s-1}\right) \rightarrow H_{k}\left(\mathfrak{n}, V_{s}\right) \rightarrow H_{k}\left(\mathfrak{n}, V_{s} / V_{s-1}\right) \rightarrow H_{k-1}\left(\mathfrak{n}, V_{s-1}\right) \rightarrow \cdots
$$

By (6.38), for $0 \leqslant i \leqslant \operatorname{dim} \mathfrak{p}_{\mathfrak{m}}$ and $0 \leqslant s \leqslant N_{0}$, we have

$$
\begin{aligned}
& \sum_{k=0}^{2 \ell}(-1)^{k} \operatorname{dim}\left(\Lambda^{i}\left(\mathfrak{p}_{\mathfrak{m}}^{*}\right) \otimes H_{k}\left(\mathfrak{n}, V_{s} / V_{s-1}\right) \otimes E_{\eta}^{ \pm}\right)^{K_{M}} \\
&=\sum_{k=0}^{2 \ell}(-1)^{k}\left\{\operatorname{dim}\left(\Lambda^{i}\left(\mathfrak{p}_{\mathfrak{m}}^{*}\right) \otimes H_{k}\left(\mathfrak{n}, V_{s}\right) \otimes E_{\eta}^{ \pm}\right)^{K_{M}}\right. \\
&\left.\quad-\operatorname{dim}\left(\Lambda^{i}\left(\mathfrak{p}_{\mathfrak{m}}^{*}\right) \otimes H_{k}\left(\mathfrak{n}, V_{s-1}\right) \otimes E_{\eta}^{ \pm}\right)^{K_{M}}\right\}
\end{aligned}
$$

By (6.35), (6.37), and (6.39), we get (6.33).

Recall that for $0 \leqslant j \leqslant 2 \ell, \eta_{j}$ is the adjoint representation of $M$ on $\Lambda^{j}\left(\mathfrak{n}_{\mathbf{C}}^{*}\right)$. In Section 5.4.2, we have seen that $\eta_{j}$ satisfies Assumption 5.4. 
Proposition 6.10. If $V$ is an irreducible Harish-Chandra $\left(\mathfrak{g}_{\mathbf{C}}, K\right)$-module with infinitesimal character $\chi$ such that $\chi\left(C^{\mathfrak{g}}\right)=0$ and

$$
\bigoplus_{\substack{0 \leqslant i \leqslant d i m \\ 0 \leqslant k \leqslant 2 \ell}} H^{i}\left(\mathfrak{m}, K_{M} ; H_{k}(\mathfrak{n}, V) \otimes \Lambda^{j}\left(\mathfrak{n}_{\mathbf{C}}^{*}\right)\right) \neq 0,
$$

then

$$
\chi=\chi_{\mathbf{1}}
$$

Proof. The proof given in [Sh18, Proposition 8.17] where $V$ is unitary extends to any irreducible Harish-Chandra $\left(\mathfrak{g}_{\mathbf{C}}, K\right)$-module without any change.

Corollary 6.11. If $V$ is Harish-Chandra $\left(\mathfrak{g}_{\mathbf{c}}, K\right)$-module with generalised infinitesimal character $\chi$ such that $\chi\left(C^{\mathfrak{g}}\right)=0$ and

$$
\sum_{\substack{0 \leqslant i \leqslant d i m \\ 0 \leqslant k \leqslant 2 \ell}}(-1)^{i+k} \operatorname{dim} H^{i}\left(\mathfrak{m}, K_{M} ; H_{k}(\mathfrak{n}, V) \otimes \Lambda^{j}\left(\mathfrak{n}_{\mathbf{C}}^{*}\right)\right) \neq 0
$$

then

$$
\chi=\chi_{\mathbf{1}}
$$

Proof. If $V$ has a composition series (6.3), by (6.39) and the Euler formula [Sh18, (8-85)], we have

$$
\begin{aligned}
\sum_{\substack{0 \leqslant i \leqslant d i m \mathfrak{m} \mathfrak{p}_{\mathfrak{m}} \\
0 \leqslant k \leqslant 2 \ell}}(-1)^{i+k} & \operatorname{dim} H^{i}\left(\mathfrak{m}, K_{M} ; H_{k}(\mathfrak{n}, V) \otimes \Lambda^{j}\left(\mathfrak{n}_{\mathbf{C}}^{*}\right)\right) \\
= & \sum_{s=0}^{N_{0}} \sum_{\substack{0 \leqslant i \leqslant d i m \mathfrak{p}_{\mathfrak{m}} \\
0 \leqslant k \leqslant 2 \ell}}(-1)^{i+k} \operatorname{dim} H^{i}\left(\mathfrak{m}, K_{M} ; H_{k}\left(\mathfrak{n}, V_{s} / V_{s-1}\right) \otimes \Lambda^{j}\left(\mathfrak{n}_{\mathbf{C}}^{*}\right)\right)
\end{aligned}
$$

By (6.42), (6.44), there is $0 \leqslant s \leqslant N_{0}$ such that

$$
\bigoplus_{\substack{0 \leqslant i \leqslant \operatorname{dim} \mathfrak{p}_{\mathfrak{m}} \\ 0 \leqslant k \leqslant 2 \ell}} H^{i}\left(\mathfrak{m}, K_{M} ; H_{k}\left(\mathfrak{n}, V_{s} / V_{s-1}\right) \otimes \Lambda^{j}\left(\mathfrak{n}_{\mathbf{C}}^{*}\right)\right) \neq 0 .
$$

By Proposition 6.10, we get (6.43).

We have a generalisation of [Sh18, Corollary 8.18].

Theorem 6.12. For $0 \leqslant j \leqslant 2 \ell$, we have

$$
r_{j}=\frac{1}{\chi\left(K / K_{M}\right)} \sum_{\substack{0 \leqslant i \leqslant \operatorname{dim} \mathfrak{p}_{\mathfrak{m}} \\ 0 \leqslant k \leqslant 2 \ell}}(-1)^{i+k} \operatorname{dim} H^{i}\left(\mathfrak{m}, K_{M} ; H_{k}\left(\mathfrak{n}, V_{\chi_{\mathbf{1}}}(\Gamma, \rho)\right) \otimes \Lambda^{j}\left(\mathfrak{n}_{\mathbf{C}}^{*}\right)\right) .
$$

In particular, if $V_{\chi_{1}}(\Gamma, \rho)=0$, for $0 \leqslant j \leqslant 2 \ell$, we have

$$
r_{j}=0 \text {. }
$$

Proof. This is a consequence of Proposition 6.9 and Corollary 6.11.

Remark 6.13. By (5.35), Theorems 6.8 and 6.12, we get (4.7) in the case $\delta(G)=1$ and $Z_{G}$ is compact. We complete the proof of Theorem 4.3 in full generality. 


\section{AN EXTENSION TO ORBIFOLDS}

In this section, we no longer assume that $\Gamma \subset G$ is torsion free. Then $Z=\Gamma \backslash G / K$ is a closed orbifold. The purpose of this section is to extend Theorem 4.3 to orbifolds.

This section is organised as follows. In Section 7.1, we establish Müller's Selberg trace formula for orbifolds.

In Section 7.2, we indicate the essential steps in generalising Theorem 4.3 to orbifolds.

7.1. Müller's Selberg trace formula on locally symmetric orbifolds. We use the notation in Section 2.6. Recall that $\Gamma \subset G$ is a discrete cocompact subgroup of $G$. Then, $Z=\Gamma \backslash G / K$ is a closed orbifold. Recall also that $\rho: \Gamma \rightarrow \mathrm{GL}_{r}(\mathbf{C})$ is a representation of $\Gamma$, and that $\left(\tau, E_{\tau}\right)$ is a representation of $K$. Define $F$ and $\mathscr{F}_{\tau}$ as in (1.33) and (2.25). Then $F$ is a flat orbifold vector bundle and $\mathscr{F}_{\tau}$ is a Hermitian orbifold vector bundle. As in the case where $\Gamma$ is torsion free, the Casimir operator $C^{\mathfrak{g}}$ induces a generalised Laplacian $C^{\mathfrak{g}, Z, \tau, \rho}$ acting on $C^{\infty}\left(Z, \mathscr{F}_{\tau} \otimes F\right)$.

For $\gamma \in \Gamma$, we have seen in Section 2.6 that $\gamma$ is semisimple. The group $K(\gamma)$ acts on the right on $\Gamma(\gamma) \backslash Z(\gamma)$. For $h \in \Gamma(\gamma) \backslash Z(\gamma)$, let $K(\gamma)_{h}$ be the stabiliser of $h$ in $K(\gamma)$. Since $\Gamma(\gamma) \backslash X(\gamma)$ is connected, the cardinality of a generic stabiliser $K(\gamma)_{h}$ is well defined (see [BLa99, Section 3.1]) and depends only on the conjugacy class of $\gamma$ in $\Gamma$. We denote it by $n_{[\gamma]}$. By [BLa99, (3.10)], we have

$$
\frac{\operatorname{vol}(\Gamma(\gamma) \backslash Z(\gamma))}{\operatorname{vol}(K(\gamma))}=\frac{\operatorname{vol}(\Gamma(\gamma) \backslash X(\gamma))}{n_{[\gamma]}} .
$$

By [ShY17, Proposition 5.3] and by (2.19),(2.20), we have

$$
n_{[\gamma]}=|K \cap \Gamma(\gamma) \cap Z(\mathfrak{p}(\gamma))|=|\operatorname{ker}(\Gamma(\gamma) \rightarrow \operatorname{Diffeo}(X(\gamma)))| .
$$

Recall that $\left[\Gamma_{+}\right] \subset[\Gamma]$ is the set of non elliptic conjugacy classes of $\Gamma$. For $[\gamma] \in[\Gamma]$, $B_{[\gamma]}$ is defined in (2.28). We have a generalisation of [ShY17, Theorem 5.4] and Theorem 2.8.

Theorem 7.1. There exist $c>0, C>0$ such that for $t>0$, we have

$$
\sum_{[\gamma] \in\left[\Gamma_{+}\right]} \frac{\operatorname{vol}\left(B_{[\gamma]}\right)}{n_{[\gamma]}}|\operatorname{Tr}[\rho(\gamma)]|\left|\operatorname{Tr}^{[\gamma]}\left[\exp \left(-t C^{\mathfrak{g}, X, \tau}\right)\right]\right| \leqslant C \exp \left(-\frac{c}{t}+C t\right) .
$$

For $t>0$, the following identity holds,

$$
\operatorname{Tr}\left[\exp \left(-t C^{\mathfrak{g}, Z, \tau, \rho}\right)\right]=\sum_{[\gamma] \in[\Gamma]} \frac{\operatorname{vol}\left(B_{[\gamma]}\right)}{n_{[\gamma]}} \operatorname{Tr}[\rho(\gamma)] \operatorname{Tr}^{[\gamma]}\left[\exp \left(-t C^{\mathfrak{g}, X, \tau}\right)\right] .
$$

Proof. The proof is similar to the one given in [ShY17, Theorem 5.4] and Theorem 2.8 .

By Theorem 7.1, proceeding as in Corollary 2.11, we get the following corollary.

Corollary 7.2. The statement of Corollary 2.11 holds for orbifolds. 
7.2. The Ruelle zeta functions on locally symmetric orbifolds. Let us follow [ShY17, Section 5.6]. By [ShY17, Remark 5.6, (5.59)], as in (4.1), the closed geodesics (see [GuHa06] or [ShY17, Remark 2.26]) on the orbifold $Z$ with positive length are given by

$$
\coprod_{[\gamma] \in\left[\Gamma_{+}\right]} B_{[\gamma]} .
$$

Moreover, if $[\gamma] \in\left[\Gamma_{+}\right]$, all the elements of $B_{[\gamma]}$ have the same length $\ell_{[\gamma]}>0$. Also, the group $\mathbb{S}^{1}$ acts locally freely on the orbifold $B_{[\gamma]}$ by rotation. So, $B_{[\gamma]} / \mathbb{S}^{1}$ is still a closed orbifold. Set

$$
m_{[\gamma]}=n_{[\gamma]}\left|\operatorname{ker}\left(\mathbb{S}^{1} \rightarrow \operatorname{Diffeo}\left(\mathrm{B}_{[\gamma]}\right)\right)\right| \in \mathbf{N}^{*} .
$$

Following [ShY17, Definition 5.10], for $\operatorname{Re}(\sigma) \gg 1$ large enough, we define the Ruelle dynamical zeta function by the same formula

$$
R_{\rho}(\sigma)=\exp \left(\sum_{[\gamma] \in\left[\Gamma_{+}\right]} \frac{\chi_{\text {orb }}\left(B_{[\gamma]} / \mathbb{S}^{1}\right)}{m_{[\gamma]}} \operatorname{Tr}[\rho(\gamma)] e^{-\sigma \ell_{[\gamma]}}\right),
$$

as in (4.5). By [ShY17, Proposition 5.9], the statement of Remark 4.2 still holds for orbifolds.

Theorem 7.3. The statements of Theorem 4.3 holds for orbifolds.

Proof. As previous, by Corollary 7.2, we need only consider the case $\delta(G)=1$. If $\eta$ satisfies Assumption 5.4, we can define the Selberg zeta function by the same formula (5.13) with $m_{[\gamma]}$ defined in (7.6). Using Theorem 7.1 instead of Theorem 2.8, by Remark 1.6, we can deduce that the statements of Theorem 5.10, (5.31), (5.34) still hold when $\Gamma$ is not torsion free. Moreover, all the results in Section 6 hold when $\Gamma$ is not torsion free. In this way, we get our theorem.

\section{REFERENCES}

[BHdlVa08] B. Bekka, P. de la Harpe, and A. Valette, Kazhdan's property (T), New Mathematical Monographs, vol. 11, Cambridge University Press, Cambridge, 2008. MR 2415834

[BeGeV04] N. Berline, E. Getzler, and M. Vergne, Heat kernels and Dirac operators, Grundlehren Text Editions, Springer-Verlag, Berlin, 2004, Corrected reprint of the 1992 original. MR 2273508 (2007m:58033)

[B11] J.-M. Bismut, Hypoelliptic Laplacian and orbital integrals, Annals of Mathematics Studies, vol. 177, Princeton University Press, Princeton, NJ, 2011. MR 2828080

[BGS88] J.-M. Bismut, H. Gillet, and C. Soulé, Analytic torsion and holomorphic determinant bundles. I. Bott-Chern forms and analytic torsion, Comm. Math. Phys. 115 (1988), no. 1, 49-78. MR 929146 (89g:58192a)

[BMaZ11] J.-M. Bismut, X. Ma, and W. Zhang, Opérateurs de Toeplitz et torsion analytique asymptotique, C. R. Math. Acad. Sci. Paris 349 (2011), no. 17-18, 977-981. MR 2838248 (2012k:58055)

[BMaZ17] J.-M. Bismut, X. Ma, and W. Zhang, Asymptotic torsion and Toeplitz operators, J. Inst. Math. Jussieu 16 (2017), no. 2, 223-349. MR 3615411

[BLa99] J.-M. Bismut and F. Labourie, Symplectic geometry and the Verlinde formulas, Surveys in differential geometry: differential geometry inspired by string theory, Surv. Differ. Geom., vol. 5, Int. Press, Boston, MA, 1999, pp. 97-311. MR 1772272 (2001i:53145)

[BSh19a] J.-M. Bismut and S. Shen, Intégrales orbitales semi-simples et centre de l'algèbre enveloppante, C. R. Math. Acad. Sci. Paris 357 (2019), no. 11-12, 897-906. MR 4038265 
[BSh19b] J.-M. Bismut and S. Shen, Geometric orbital integrals and the center of the enveloping algebra, arXiv: 1910.11731 (2019).

[BZ92] J.-M. Bismut and W. Zhang, An extension of a theorem by Cheeger and Müller, Astérisque (1992), no. 205, 235, With an appendix by François Laudenbach. MR 1185803 (93j:58138)

[BWSh20] Y. Borns-Weil and S. Shen, Dynamical zeta functions in the nonorientable case, arXiv:2007.08043 (2020).

[BrKa07] M. Braverman and T. Kappeler, Refined analytic torsion as an element of the determinant line, Geom. Topol. 11 (2007), 139-213. MR 2302591

[BrKa08] M. Braverman and T. Kappeler, Refined analytic torsion, J. Differential Geom. 78 (2008), no. 2, 193-267. MR 2394022

[BrDi85] T. Bröcker and T. tom Dieck, Representations of compact Lie groups, Graduate Texts in Mathematics, vol. 98, Springer-Verlag, New York, 1985. MR 781344 (86i:22023)

[BuH07] D. Burghelea and S. Haller, Complex-valued Ray-Singer torsion, J. Funct. Anal. 248 (2007), no. 1, 27-78. MR 2329682

[BuH08] D. Burghelea and S. Haller, Torsion, as a function on the space of representations, $C^{*}$-algebras and elliptic theory II, Trends Math., Birkhäuser, Basel, 2008, pp. 41-66. MR 2408135

[BuH10] D. Burghelea and S. Haller, Complex valued Ray-Singer torsion II, Math. Nachr. 283 (2010), no. 10, 1372-1402. MR 2744135

[CMil10] S.E. Cappell and E.Y. Miller, Complex-valued analytic torsion for flat bundles and for holomorphic bundles with $(1,1)$ connections, Comm. Pure Appl. Math. 63 (2010), no. 2, 133-202. MR 2588459

[Ch79] J. Cheeger, Analytic torsion and the heat equation, Ann. of Math. (2) 109 (1979), no. 2, 259-322. MR 528965 (80j:58065a)

[DGRSh20] N. V. Dang, C. Guillarmou, G. Rivière, and S. Shen, The Fried conjecture in small dimensions, Invent. Math. 220 (2020), no. 2, 525-579. MR 4081137

[DaY17] X. Dai and J. Yu, Comparison between two analytic torsions on orbifolds, Math. Z. 285 (2017), no. 3-4, 1269-1282. MR 3623749

[dR50] G. de Rham, Complexes à automorphismes et homéomorphie différentiable, Ann. Inst. Fourier Grenoble 2 (1950), 51-67 (1951). MR 0043468 (13,268c)

[DuKVa79] J. J. Duistermaat, J. A. C. Kolk, and V. S. Varadarajan, Spectra of compact locally symmetric manifolds of negative curvature, Invent. Math. 52 (1979), no. 1, 27-93. MR 532745 (82a:58050a)

[DyZw16] S. Dyatlov and M. Zworski, Dynamical zeta functions for Anosov flows via microlocal analysis, Ann. Sci. Éc. Norm. Supér. (4) 49 (2016), no. 3, 543-577. MR 3503826

[DyZw17] S. Dyatlov and M. Zworski, Ruelle zeta function at zero for surfaces, Invent. Math. 210 (2017), no. 1, 211-229. MR 3698342

[E96] P. B. Eberlein, Geometry of nonpositively curved manifolds, Chicago Lectures in Mathematics, University of Chicago Press, Chicago, IL, 1996. MR 1441541

[FaT00] M. Farber and V. Turaev, Poincaré-Reidemeister metric, Euler structures, and torsion, J. Reine Angew. Math. 520 (2000), 195-225. MR 1748274

[Fra35] W. Franz, Über die Torsion einer Überdeckung., J. Reine Angew. Math. 173 (1935), 245-254 (German).

[F86] D. Fried, Analytic torsion and closed geodesics on hyperbolic manifolds, Invent. Math. 84 (1986), no. 3, 523-540. MR 837526 (87g:58118)

[F87] D. Fried, Lefschetz formulas for flows, The Lefschetz centennial conference, Part III (Mexico City, 1984), Contemp. Math., vol. 58, Amer. Math. Soc., Providence, RI, 1987, pp. 19-69. MR 893856 (88k:58138)

[FrN15] S. Friedl and M. Nagel, 3-manifolds that can be made acyclic, Int. Math. Res. Not. IMRN (2015), no. 24, 13360-13378. MR 3436149

[GiLPo13] P. Giulietti, C. Liverani, and M. Pollicott, Anosov flows and dynamical zeta functions, Ann. of Math. (2) 178 (2013), no. 2, 687-773. MR 3071508 
[GolM88] W.M. Goldman and J.J. Millson, The deformation theory of representations of fundamental groups of compact Kähler manifolds, Inst. Hautes Études Sci. Publ. Math. (1988), no. 67, 43-96. MR 972343

[GoKr69] I. C. Gohberg and M. G. Krein, Introduction to the theory of linear nonselfadjoint operators, Translated from the Russian by A. Feinstein. Translations of Mathematical Monographs, Vol. 18, American Mathematical Society, Providence, R.I., 1969. MR 0246142

[GuHa06] K. Guruprasad and A. Haefliger, Closed geodesics on orbifolds, Topology 45 (2006), no. 3, 611-641. MR 2218759

[HeSc83] H. Hecht and W. Schmid, Characters, asymptotics and n-homology of Harish-Chandra modules, Acta Math. 151 (1983), no. 1-2, 49-151. MR 716371 (84k:22026)

[Hi74] N. Hitchin, Harmonic spinors, Advances in Math. 14 (1974), 1-55. MR 0358873 (50 \#11332)

[K86] A. W. Knapp, Representation theory of semisimple groups, Princeton Mathematical Series, vol. 36, Princeton University Press, Princeton, NJ, 1986, An overview based on examples. MR 855239 (87j:22022)

[K02] A. W. Knapp, Lie groups beyond an introduction, second ed., Progress in Mathematics, vol. 140, Birkhäuser Boston, Inc., Boston, MA, 2002. MR 1920389 (2003c:22001)

[Ma05] X. Ma, Orbifolds and analytic torsions, Trans. Amer. Math. Soc. 357 (2005), no. 6, 2205-2233 (electronic). MR 2140438

[Ma19] X. Ma, Geometric hypoelliptic Laplacian and orbital integrals [after Bismut, Lebeau and Shen], no. 407, 2019, Séminaire Bourbaki. Vol. 2016/2017. Exposés 1120-1135, pp. Exp. No. 1130, 333-389. MR 3939281

[MaMar07] X. Ma and G. Marinescu, Holomorphic Morse inequalities and Bergman kernels, Progress in Mathematics, vol. 254, Birkhäuser Verlag, Basel, 2007. MR 2339952 (2008g:32030)

[MaMar15] X. Ma and G. Marinescu, Exponential estimate for the asymptotics of Bergman kernels, Math. Ann. 362 (2015), no. 3-4, 1327-1347. MR 3368102

[Mi68a] J. Milnor, Infinite cyclic coverings, Conference on the Topology of Manifolds (Michigan State Univ., E. Lansing, Mich., 1967), Prindle, Weber \& Schmidt, Boston, Mass., 1968, pp. 115-133. MR 0242163 (39 \#3497)

[Mi68b] J. Milnor, A note on curvature and fundamental group, J. Differential Geometry 2 (1968), 1-7. MR 0232311

[MoSt91] H. Moscovici and R. J. Stanton, $R$-torsion and zeta functions for locally symmetric manifolds, Invent. Math. 105 (1991), no. 1, 185-216. MR 1109626 (92i:58199)

[Mü78] W. Müller, Analytic torsion and R-torsion of Riemannian manifolds, Adv. in Math. 28 (1978), no. 3, 233-305. MR 498252 (80j:58065b)

[Mü93] W. Müller, Analytic torsion and R-torsion for unimodular representations, J. Amer. Math. Soc. 6 (1993), no. 3, 721-753. MR 1189689 (93m:58119)

[Mü11] W. Müller, A Selberg trace formula for non-unitary twists, Int. Math. Res. Not. IMRN (2011), no. 9, 2068-2109. MR 2806558

[Mü20] W. Müller, On Fried's conjecture for compact hyperbolic manifolds, arXiv:2005.01450 (2020).

[MüPf13] W. Müller and J. Pfaff, Analytic torsion and $L^{2}$-torsion of compact locally symmetric manifolds, J. Differential Geom. 95 (2013), no. 1, 71-119. MR 3128980

[Pa83] A. Pazy, Semigroups of linear operators and applications to partial differential equations, Applied Mathematical Sciences, vol. 44, Springer-Verlag, New York, 1983. MR 710486

[Q85] D. Quillen, Superconnections and the Chern character, Topology 24 (1985), no. 1, 8995. MR 790678 (86m:58010)

[RaSi71] D. B. Ray and I. M. Singer, R-torsion and the Laplacian on Riemannian manifolds, Advances in Math. 7 (1971), 145-210. MR 0295381 (45 \#4447)

[Rap99] A. Rapinchuk, On the finite-dimensional unitary representations of Kazhdan groups, Proc. Amer. Math. Soc. 127 (1999), no. 5, 1557-1562. MR 1476387 
[Re35] K. Reidemeister, Homotopieringe und Linsenräume, Abh. Math. Sem. Univ. Hamburg 11 (1935), no. 1, 102-109. MR 3069647

[SR99] S. A. Salamanca-Riba, On the unitary dual of real reductive Lie groups and the $A_{\mathfrak{q}}(\lambda)$ modules: the strongly regular case, Duke Math. J. 96 (1999), no. 3, 521-546. MR 1671213 (2000a:22023)

[Sa57] I. Satake, The Gauss-Bonnet theorem for V-manifolds, J. Math. Soc. Japan 9 (1957), 464-492. MR 0095520 (20 \#2022)

[Se56] A. Selberg, Harmonic analysis and discontinuous groups in weakly symmetric Riemannian spaces with applications to Dirichlet series, J. Indian Math. Soc. (N.S.) 20 (1956), 47-87. MR 0088511

[Se60] A. Selberg, On discontinuous groups in higher-dimensional symmetric spaces, Contributions to function theory (internat. Colloq. Function Theory, Bombay, 1960), Tata Institute of Fundamental Research, Bombay, 1960, pp. 147-164. MR 0130324

[Sh18] S. Shen, Analytic torsion, dynamical zeta functions, and the Fried conjecture, Anal. PDE 11 (2018), no. 1, 1-74. MR 3707290

[Sh19] S. Shen, Analytic torsion and dynamical flow: a survey on the Fried conjecture.

[ShY17] S. Shen and J. Yu, Flat vector bundles and analytic torsion on orbifolds, arXiv: 1704.08369 (2017). To appear in Comm. Anal. Geom.

[ShY18] S. Shen and J. Yu, Morse-Smale flow, Milnor metric, and dynamical zeta function, arXiv: 1806.00662 (2018).

[S01] M. A. Shubin, Pseudodifferential operators and spectral theory, second ed., SpringerVerlag, Berlin, 2001, Translated from the 1978 Russian original by Stig I. Andersson. MR 1852334

[Sp18] P. Spilioti, Selberg and Ruelle zeta functions for non-unitary twists, Ann. Global Anal. Geom. 53 (2018), no. 2, 151-203. MR 3766581

[Sp20a] P. Spilioti, The functional equations of the Selberg and Ruelle zeta functions for nonunitary twists, arXiv:1507.05947. To appear Ann. Global Anal. Geom. (2020)

[Sp20b] P. Spilioti, Twisted Ruelle zeta function and complex-valued analytic torsion, arXiv:2004.13474 (2020).

[SuZ08] G. Su and W. Zhang, A Cheeger-Müller theorem for symmetric bilinear torsions, Chin. Ann. Math. Ser. B 29 (2008), no. 4, 385-424. MR 2429629

[T89] V. G. Turaev, Euler structures, nonsingular vector fields, and Reidemeister-type torsions, Izv. Akad. Nauk SSSR Ser. Mat. 53 (1989), no. 3, 607-643, 672. MR 1013714

[Vo84] D. A. Vogan, Jr., Unitarizability of certain series of representations, Ann. of Math. (2) 120 (1984), no. 1, 141-187. MR 750719 (86h:22028)

[VoZu84] D. A. Vogan, Jr. and G. J. Zuckerman, Unitary representations with nonzero cohomology, Compositio Math. 53 (1984), no. 1, 51-90. MR 762307 (86k:22040)

[W82] E. Witten, Supersymmetry and Morse theory, J. Differential Geom. 17 (1982), no. 4, 661-692 (1983). MR 683171 (84b:58111)

[Yo95] K. Yosida, Functional analysis, Classics in Mathematics, Springer-Verlag, Berlin, 1995, Reprint of the sixth (1980) edition. MR 1336382 (96a:46001)

Institut de Mathématiques de Jussieu-Paris Rive Gauche, Sorbonne Université, 4 Place Jussieu, 75252 Paris Cedex 5, France.

E-mail address: shu. shen@imj-prg.fr 